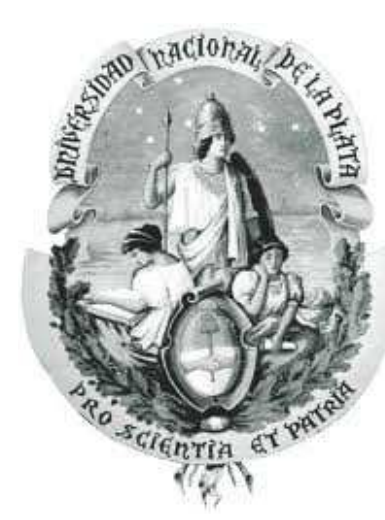

Universidad Nacional de La Plata

Facultad de Ciencias Astronómicas y Geofísicas

\title{
Violación DEL PRINCIPIO DE EQUIVALENCIA EN TEORÍAS CON $\alpha$ VARIABLE
}

Tesis Presentada Por Lic. Lucila Kraiselburd PARA OBTENER EL GRADO DE DOCTOR EN Astronomía

Director: Dr. HÉctor Vucetich

Grupo de Astrofísica, Relatividad y Cosmología

Marzo, 2012 


\section{Resumen de la tesis}

En esta tesis presentamos un estudio de la variación de la constante de estructura fina $\alpha$. Más precisamente, analizamos el modelo propuesto por Bekenstein y lo comparamos tanto con experimentos tipo Eötvös, que verifican al Principio de Equivalencia, como con mecanismos térmicos de la Tierra y de otros planetas del Sistema Solar. Además, establecemos cotas superiores a los "parámetros de violación" del Principio de Equivalencia debido a la acción de las fuerzas fundamentales. Estos parámetros se encuentran estrechamente relacionados con muchas de las constantes fundamentales, por lo tanto a partir de ellos se pueden determinar límites superiores a las posibles variaciones espaciales de las constantes. En este caso, se eligió acotar las variaciones de cuatro constantes: $\alpha, \Lambda_{Q C D}, \sin ^{2} \theta_{W}$ y $G_{F}$, para luego poder acotar las variaciones de las constantes de acoplamiento de Gauge $\alpha_{1}, \alpha_{2}, \alpha_{3}$, el valor de expectación del Higgs $v$, y las de las masas de partículas elementales.

Estudiamos en profundidad la teoría expuesta por Bekenstein, quien propone la variación de $\alpha$ por la introducción de un campo escalar adimensional " $\psi$ " generando variaciones en las cargas. Utiliza hipótesis muy generales de la física tales como: la covarianza, la invarianza de Gauge, la causalidad y la invarianza ante la reversibilidad del tiempo del electromagnetismo. La propia generalidad de estos supuestos permite garantizar la aplicabilidad del régimen a otro "gauge" de interacciones, como las interacciones fuertes. Además, introduce otra simplificación muy útil, dado que la gravitación del sector no se ve afectada por el campo escalar introducido para variar la constante de acoplamiento. Bekenstein demostró que en su teoría, usando partículas clásicas estáticas como modelo de materia, no se produce una aceleración anómala, y por lo tanto, se cumplen la Universalidad de Caída Libre y el Principio de Equivalencia mencionados anteriormente. Tanto los fenómenos cuánticos como 
los efectos que pueden ocurrir a escalas de altas energías y/o pequeñas escalas de distancias no han sido tomados en cuenta en esta teoría ya que es descripta en forma clásica.

Para esta tesis nos propusimos trabajar con un modelo de materia más realista. Por eso, estudiamos también en forma exhaustiva las técnicas desarrolladas por

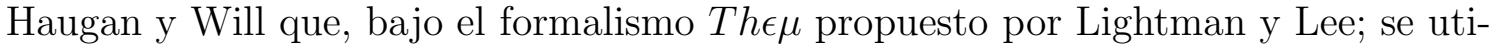
lizan para analizar el comportamiento de cuerpos que interactúan electromagnéticamente en caída libre, analizando toda clase de teorías gravitatorias (las consideradas "métricas" y las "no métricas"). A órdenes "post-post-Coulombiano", se encuentran violaciones al Principio de Equivalencia dependientes de la estructura magnética de los cuerpos utilizados en los experimentos.

Las modificaciones hechas fueron las siguientes: trabajamos en el límite no relativista pero utilizando la expresión total del campo electromagnético para los momentos; tomamos al sistema como semiclásico donde los campos son considerados clásicos pero las partículas responden a un modelo del tipo Thomas-Fermi; supusimos la presencia de campos externos (el campo dilatónico ya mencionado " $\psi$ ", y el potencial newtoniano estará representado por " $\phi_{N}$ ") despreciando las contribuciones de los campos autogenerados.

Nuestros descubrimientos fueron los siguientes:

- Partiendo de las ecuaciones encontradas por Bekenstein mostramos que, para un potencial electrostático producido por una densidad arbitraria de carga pero acotada utilizando nuestro modelo de partícula, no podemos descartar ni reafirmar el resultado expuesto por Bekenstein (donde se cancelan las contribuciones eléctricas con contribuciones provenientes de la masa). Por lo tanto, seremos pesimistas y supondremos como válida dicha afirmación. Igualmente, en un modelo cuántico de materia, las fluctuaciones cuánticas en la densidad número de partículas generan campos magnéticos. Demostramos que estas contribuciones en la energía magnética son las responsables de la aparición de un término anómalo en la aceleración de un sistema de partículas que interactúa con los campos ya mencionados que, a la hora de testear con los experimentos de Eötvös, nos hace rechazar a la teoría.

- Analizamos también el comportamiento de la materia y de los campos que 
actúan con el sistema. Debido a la interacción con el campo propuesto por Bekenstein, se genera un flujo "mágico" de energía, encontrando cotas estrictas para la variación temporal de $\alpha$ a partir de la comparación del modelo con datos termodinámicos astronómicos y geofísicos. Estas cotas son generales ya que no dependen del modelo de variación de $\alpha$.

- Por último, establecemos límites independientes a las variaciones espaciales de $\alpha, \Lambda_{Q C D}, \sin ^{2} \theta_{W}$ y $G_{F}$ realizando un ajuste con los últimos y más precisos datos provenientes de experimentos de Eötvös. También, ponemos restricciones a las variaciones espaciales de: constantes de acoplamiento de Gauge, el valor de expectación del Higgs y las de las masas de partículas elementales. Para lograr esto utilizamos: los resultados de la "teoría de perturbación quiral", por ende, las masas de los quarks livianos son muy pequeñas, pero no cero; desarrollos en serie; siempre sin olvidar que en el límite quiral, cualquier cantidad de dimensiones de masa o energía debe ser proporcional a $\Lambda_{Q C D}$. 


\section{Agradecimientos}

Quiero agradecerles a las autoridades del Conicet y del Observatorio por haberme dado la posibilidad de tener un apoyo financiero y un lugar de trabajo durante estos cinco años. También a los miembros del jurado que evaluaron esta tesis, en especial al Dr. Osvaldo Civitarese por sus sugerencias y comentarios.

Estoy profundamente agradecida con mi director, el Dr. Héctor Vucetich, que no sólo ha sido y es un maestro y guía en el ámbito acádemico, sino además me enseña día a día cosas nuevas sobre la vida. Fue y es un placer trabajar con él.

Agradezco a los Dres. Pablo Sisterna y Marcelo Miller Bertolami, quienes trabajaron con nosotros para llevar a cabo parte de esta tesis, y a los miembros del grupo de Gravitación, Astrófisica y Cosmología: Susana Landau, Milva Orsaria, Claudia Scóccola, Ignacio Ranea Sandoval y Gustavo Contreras, con los que compartí muchos momentos en todo este tiempo. Mis compañeras de oficina, Florencia Teppa Pannia y Mercedes Mosquera, se merecen un reconocimiento especial por haberme brindado su apoyo desde el primer día, ya sea desde unas palabras de aliento en algún momento crítico, hasta el préstamo de libros o ayuda con alguna cuenta y/o programa de computación.

Gracias a mis amigos tanto del Observatorio como de los de la vida por la compañía y comprensión todos estos años; y a mi familia: abuelas y tías quienes siempre se interesaron por saber de mis progresos; mis hermanos, con sus palabras de aliento; y en especial a mis padres, que me acopañaron a transitar este camino y sin ellos no hubiese llegado a ser quien soy.

Finalmente, quiero darle las gracias a Martín Marchetti que me acompaña todos los días y trata de entenderme, cosa que no es para nada fácil. 


\section{Índice general}

Resumen de la tesis $\quad 2$

$\begin{array}{ll}\text { Agradecimientos } & 5\end{array}$

1. Introducción $\quad 8$

2. Modelo teórico de Bekenstein para la variación de $\alpha \quad 13$

2.1. Hipótesis utilizadas en el desarrollo de la teoría . . . . . . . . . . . 13

2.2. Desarrollo de la dinámica del modelo . . . . . . . . . . . . . . . . . . 14

2.3. El campo $\epsilon$ producido por una carga puntual en reposo . . . . . . . . 17

2.4. Las variaciones temporales de $\alpha \ldots \ldots$. . . . . . . . . 17

2.5. Las variaciones espaciales de $\alpha \ldots \ldots$. . . . . . . . . . . . . 19

2.5.1. Modelo para una carga puntual . . . . . . . . . . . . . . 20

2.5.2. Modelo para un sistema de cargas . . . . . . . . . . . . . 21

2.5.3. Modelo para una conjunto de dipolos magnéticos . . . . . . . 23

2.5.4. Conclusiones .................. . . 25

3. El modelo de Bekenstein para el caso semiclásico 26

3.1. Desarrollo del modelo . . . . . . . . . . . . . . . . . . . . . . . 26

3.1.1. Modelo de Thomas-Fermi cuántico . . . . . . . . . . . . . . . 30

3.2. Movimiento de un cuerpo en el campo $\psi$. . . . . . . . . . . . . . . . 33

3.2.1. Comportamiento de la materia . . . . . . . . . . . . . 36

3.2.2. Contribución del campo magnético al campo escalar . . . . . . 41

3.3. Resultados . . . . . . . . . . . . . . . . . . . . . 45 
4. Intercambio de energía en la teoría de Bekenstein 48

4.1. Energía transferida . . . . . . . . . . . . . . . . 50

4.1.1. La energía electromagnética de la materia . . . . . . . . . . 54

4.2. Flujos de calor planetarios . . . . . . . . . . . . . . . . . 55

4.2.1. Flujo de calor terrestre . . . . . . . . . . . . . . . 55

4.2.2. Flujos de calor de planetas exteriores . . . . . . . . . . . 58

4.3. Resultados . . . . . . . . . . . . . . . . . . 60

5. Límites para las interacciones fundamentales y acoplamientos $\quad 62$

5.1. El Principio de Equivalencia y los experimentos Eötvös . . . . . . . . 62

5.2. Parámetros de violación $\Gamma$ a partir de las interacciones fundamentales 65

5.2.1. Aportes de las diferencias de masa y de las energías de ligadura 65

5.2.2. Contraste entre la teoría y las observaciones . . . . . . . . . 69

5.2.3. Refinamiento de los parámetros $\Gamma$. . . . . . . . . . . 71

5.3. Límites para las variaciones espaciales de las constantes fundamentales 72

5.3.1. La Teoría Perturbativa Quiral . . . . . . . . . . . . . . . 72

5.3.2. Variaciones espaciales de las constantes fundamentales . . . . 76

5.3.3. Relación entre los $\Gamma$ y las variaciones de las constantes . . . . 78

5.4. Variaciones de las constantes de acoplamiento y el VEV del Higgs . 79

5.4.1. Variaciones de masas elementales . . . . . . . . . . . . . . . 80

$\begin{array}{lr}\text { 6. Conclusiones } & 84\end{array}$

A. Tratamiento semiclásico del modelo de Thomas -Fermi $\quad 87$

A.1. Ecuación de Thomas-Fermi . . . . . . . . . . . . . . . . . . . . 90

$\begin{array}{ll}\text { Bibliografía } & 92\end{array}$ 


\section{Capítulo 1}

\section{Introducción}

Desde sus comienzos el hombre ha tratado de explicar los fenómenos que ocurren a su alrededor. Con el paso del tiempo, fue adquiriendo nuevas estructuras de pensamiento ayudado con el desarrollo de tecnologías para dicho fin. En la actualidad, las dos teorías que mejor describen al Universo son la Relatividad General (GR), estudiando la fuerza gravitatoria, y el Modelo Standard (SM), que analiza y unifica las fuerzas electromagnética, débil y fuerte. Gracias a ellas, se puede obtener una buena descripción a bajas energías de la naturaleza mediante la utilización de ciertos parámetros que tienen la particularidad de permanecer invariantes frente a cambios en el espacio-tiempo y en el sistema de referencia. Éstos además, no son determinados por la teoría sino que se obtienen mediante la experimentación, son las "Constantes Fundamentales".

A mediados del siglo XX, Dirac [43, 44] llegó a la conclusión que no podía ser casualidad que el orden de magnitud del cociente entre las fuerzas electromagnética y gravitatoria entre un protón y un electrón coincidiera con la razón entre la edad del Universo y el tiempo que tarda la luz en atravesar un átomo de Hidrógeno $\left(\sim 10^{40}\right)$. Él postuló lo siguiente, dado que el tamaño del Universo visible aumenta con el transcurrir del tiempo, el gran número " 10 " tendría que crecer al mismo ritmo; por lo cual, sería necesario que se la intensidad de la fuerza gravitatoria decayera para que también creciera el cociente entre las fuerzas electromagnética y gravitatoria de manera tal de mantener la coincidencia, y abandonando así la constancia de la constante de gravitación de Newton, $G_{N}$. Pero, su hipótesis quedó refutada en 1948 
cuando Teller [132] calculó que si la gravedad hubiera sido mayor en otro tiempo, la Tierra habría estado muy cerca del Sol en el pasado y no podría presentar entonces ahora sus características actuales. Más adelante, Gamow [61] intentó revivir la idea de Dirac, argumentando que también podía ocurrir que la carga del electrón $e$ variase en el tiempo como requiere la ecuación, dejando intacta la constante $G_{N}$. Luego a fines de la década del 60, se comprobó mediante observaciones hechas en quásares [48], y el análisis de abundancias en meteoritos de emisiones $\beta$ de larga vida [10], que la carga del electrón no había cambiado, dado que en caso contrario las reacciones nucleares habrían sido distintas.

Desde hace ya mucho tiempo, la física ha tratado de construir una única teoría que unifique las cuatro interacciones fundamentales. Hasta el momento, se ha podido relacionar tres de ellas (electromagnética, débil y fuerte), pero no se ha encontrado un teoría cuántica de la gravedad. Existen muchas teorías que tratan de lograr dicha unificación como por ejemplo: las teorías de múltiples dimensiones, propuestas por Kaluza, Klein, Chodos y Detweiler y Weinberg entre otros[24, 73, 76, 142]; las teorías de Supercuerdas [35, 36, 94]; y las teorías de Brana [147]. En todas ellas, las energías involucradas en los fénomenos son muy altas haciéndose dificultosas las verificaciones de validez. Sin embargo, algunas de sus hipótesis y consecuencias pueden ser estudiadas a bajas energías a través de experimentos y observaciones. Todas ellas predicen la variación en el espacio-tiempo de alguna y/o todas las constantes fundamentales de distintas maneras según el modelo.

También existen otras teorías efectivas a bajas energías que sugieren y describen una posible variación de las constantes fundamentales. Éstas proponen asociar el valor de las constantes a un campo en el espacio-tiempo tal que; el Lagrangiano del sistema cumpla con principios generales, y se puedan comparar las soluciones que se desprenden de sus ecuaciones de movimiento con datos observacionales. Algunos de estos modelos son: el propuesto por Bekenstein [15, 16] para estudiar las variación espacio-temporal de la constante de estructura fina $\alpha$; Barrows y Magueijo analizan una posible variación de la masa del electrón $m_{e}$ [12]; Albrecht y Magueijo sugieren un modelo con velocidad de la luz $c$ variable [6], otros autores amplian este modelo $[26-28,74]$; y otros como Brax y Davis [20] predicen un cambio en la constante $G_{N}$.

Para poder determinar la veracidad de estas teorías y también establecer res- 
tricciones a los posibles cambios en las constantes fundamentales, es necesario el aporte de métodos tanto experimentales como observacionales, que abarcan desde mediciones de laboratorio, hasta datos de origen cosmológico. Algunos de ellos son: el análisis de la abundancia de decaimientos $\beta$ de vida media larga en meteoritos y minerales geológicos [48, 126, 127]; el reactor nuclear natural que operó en Oklo hace aproximadamente $1,8 \times 10^{9}$ años [34, 58]; la comparación entre las frecuencias de relojes atómicos de distinto número atómico [115, 128]; el análisis de espectros de quásares de alto corrimiento al rojo [9, 92, 103, 104, 138, 140]; y el análisis de datos obtenidos a partir de la radiación cósmica de fondo [13, 87, 123] y de la abundancia de ${ }^{4} \mathrm{He}$ producida en la nucleosíntesis primordial [17, 88, 89, 101]. Si bien la mayoría de estos experimentos y observaciones concuerdan con que la variación de la constantes fundamentales es nula, en 1999 y en 2009, Webb y colaboradores han mostrado en su análisis una significativa variación tanto de $\alpha$ como de $\mu=m_{p} / m_{e}$ [103, 138-140] y esto es una muy buena motivación para seguir investigando el tema.

Otra interesante forma de verificar estas teorías es analizar la posible violación del Principio de Equivalencia Débil (WEP) debido a variaciones espaciales de las constantes fundamentales [100], que es un hecho fácil de probar mediante el Principio de Conservación de la Energía [64]. El Principio de Equivalencia Débil (WEP) establece que la línea de Universo de un cuerpo inmerso en un campo gravitatorio es independiente de su composición y estructura [100, 145], es una generalización de la "Ley de Galileo de la Universalidad de la Caída Libre". Dado que la Relatividad General tiene como una de sus consecuencias al Principio de Equivalencia, las pruebas de certificación de su validez son muy importantes a la hora de "intentar una nueva física". Hasta el momento, las pruebas más precisas han sido los experimentos del tipo Eötvös, los cuales testean la igualdad de la aceleración de cuerpos de diferente composición y/o estructura [49, 100, 141]. Muchos experimentos para mejorar su precisión se han estado desarrollando desde la mitad del siglo XX hasta la actualidad [19, 119, 122, 130], siendo de gran utilidad para imponer límites estrictos a los parámetros que describen violaciones al Principio de Equivalencia Débil[11, 22, 40, 131, 145].

En este trabajo estudiamos en profundidad las teorías de Bekenstein [15, 16], y las modificamos tomando un modelo de partícula un poco más complejo, para luego 
comparar con experimentos de Eötvös y así comprobar su validez [79]. Analizamos las ecuaciones que rigen el movimiento de la materia así como también el intercambio de energía entre ella, el campo escalar debido al "nuevo acoplamiento" y el campo electromagnético encontrando cómo el flujo de energía de la materia es modificado por el campo escalar. Del estudio de la historia térmica de la Tierra y otros planetas, en la presencia del campo escalar propuesto por Bekenstein, obtenemos estrictas cotas [78]. Además, nos interesamos en estudiar los parámetros que describen violaciones al Principio de Equivalencia utilizando los últimos datos obtenidos a partir de experimentos Eötvös. Así, esta tesis se encuentra estructurada de la siguiente manera:

- En el capítulo 2 presentamos una detallada descripción de los modelos propuestos por Bekenstein [15, 16].

- En el capítulo 3 desarrollamos nuestras modificaciones añadidas al modelo y estudiamos tanto las supuestas fuerzas generadas como las contribuciones de los campos eléctrico y magnético al campo escalar. Los aportes de dichos campos son estudiados por separado, poniendo una especial atención en el cálculo de la energía magnética .

- En el capítulo 4 discutimos intercambio de energía entre la materia y los campos escalar y electromagnético produciendo cambios en su flujo. Este último puede considerarse una cota independiente a la hora de testear las variaciones temporales de la constante de estructura fina $\alpha$.

- En el capítulo 5 realizamos un análisis de las posibles causas que producen violaciones al Principio de Equivalencia, las cuales pueden ser verificadas con mucha precisión mediante datos provenientes de experimentos tipo Eötvös. Reajustamos los "parámetros de violación" para las fuerzas electromagnética, débil y fuerte calculados por Chamoun y Vucetich [22] con nuevos datos experimentales. Establecemos además restricciones a las posibles variaciones espaciales de algunas de las constantes fundamentales $\alpha, \Lambda_{Q C D}, \sin ^{2} \theta_{W}$ y $G_{F}$; así como también a las variciones espaciales de las constantes de acoplamiento $\alpha_{1}, \alpha_{2}, \alpha_{3}$ y el valor de expectación del Higgs $v$, y las masas de partículas 
elementales $M_{Z}, M_{W}, m_{q}$ y $m_{l}$. Todo esto utilizando resultados de la "Teoría Perturbativa Quiral".

- Por último, en el capítulo 6 mostraremos nuestras conclusiones.

Esta tesis está basada en los siguientes artículos:

- Violation of the Weak Equivalence Principle in Bekenstein's theory, [79].

- Energy production in varying a theories, [78].

- Constraining the fundamental interactions and couplings with Eötvös experiments, [80].

- General statements from Bekenstein's theory, [81]. 


\section{Capítulo 2}

\section{Modelo teórico de Bekenstein para la variación de $\alpha$}

En este capítulo explicaremos el modelo propuesto por Bekenstein [15, 16] para la variación de la constante de estructura fina $\alpha$. Esta teoría forma parte del grupo de teorías efectivas a bajas energías, las cuales no predicen la variación simultánea temporal y/o espacial de las constantes de acoplamiento [6, 12, 20], como sí lo hacen aquellos desarrollos teóricos que intentan unificar las cuatro interacciones fundamentales [24, 35, 36, 73, 76, 142].

Aquí, se reemplaza la constante cuya variación se desea estudiar, por un campo escalar que será el responsable de los cambios.

\subsection{Hipótesis utilizadas en el desarrollo de la teoría}

La constante de estructura fina $\alpha$ se la puede considerar como el límite a bajas energías de la constante de acoplamiento electromagnético (renormalizada). La teoría de campos general utilizada en este trabajo, está basada en ocho hipótesis:

1. Para $\alpha$ constante, el electromagnetismo se reduce al de Maxwell, y el acoplamiento del vector potencial con la materia es mínimo.

2. Las variaciones en $\alpha$ resultan de la dinámica. Se rechazan las leyes de violación ya escritas. Si $\alpha$ variara, su cambio se verá reflejado en la materia cargada. 
Luego ésta deberá influenciar a $\alpha$.

3. Tanto la acción de $\alpha$ como la de la electrodinámica son invariantes relativistas.

4. La acción es invariante de Gauge localmente.

5. El electromagnetismo es causal.

6. El electromagnetismo es invariante ante la reversibilidad del tiempo.

7. Toda escala de longitud en la teoría no puede ser menor a la longitud de Planck $\ell_{P}=\left[\frac{G \hbar}{c^{3}}\right]^{\frac{1}{2}}$. Para probar experimentalmente las teorías donde las escalas de $\ell_{B}$ son menores, se necesitan partículas con una energía $E \approx c \hbar / \ell_{B}$. Si $\ell_{B}<\ell_{P}$, la energía sería tan grande que el radio gravitacional $\frac{2 G E}{c^{4}}$ excedería a $\ell$. Por lo cual, la partícula probablemente caería en un agujero negro que ella misma produjo, frustrando así cualquier intención de generar una teoría mediante el estudio de su dispersión. ${ }^{1}$

8. La gravitación está descripta por una métrica del espacio-tiempo que satisface las ecuaciones de Einstein.

\subsection{Desarrollo de la dinámica del modelo}

El único parámetro que se tomará como variable en $\alpha=\frac{e^{2}}{\hbar c}$ es la carga. Por lo tanto, en el caso en donde todas las cargas $e_{i}$ de un sistema varían al unísolo, $e_{i}=e_{i 0} \epsilon\left(x^{\mu}\right)$, donde $e_{i 0}$ representa al acoplamiento constante de la partícula $i, \mathrm{y}$ $\epsilon\left(x^{\mu}\right)$ es un campo escalar adimensional cuya dinámica debe ser invariante ante un reescaleo del campo.

Se considera la dinámica clásica de una partícula puntual en reposo de masa $m$ y carga $e_{0} \epsilon$ en el espacio tiempo plano, siendo la convención de signos $\{-,+,+,+\}$.

\footnotetext{
${ }^{1}$ Esta última afirmación no debe ser tomada en cuenta si la teoría es considerada como límite a bajas energías de "teorías del todo" (TOE), ya que en estos casos se introducen otras escalas de longitud fundamentales. En efecto, hay una otra escala de longitud según la teoría de cuerdas, el "parámetro de tensión", de modo que es posible acotar cualquier longitud con esta "nueva" escala de longitud que es más pequeña que $\ell_{P}$.
} 
El lagrangiano de este sistema es,

$$
L=-m c\left(-u^{v} u_{v}\right)^{\frac{1}{2}}+\left(\frac{e_{0} \epsilon}{c}\right) u^{v} A_{v} \quad u^{v}=\frac{d x^{v}}{d \tau},
$$

el cual sólo cambia mediante la derivada perfecta $\left(e_{0} / c\right) d \chi / d \tau$ dada la hipótesis 2 . De esta manera,

$$
\begin{gathered}
S=\int L d t, \\
\epsilon=\left(\frac{\alpha}{\alpha_{0}}\right)^{\frac{1}{2}} .
\end{gathered}
$$

$\epsilon$ se encuentra en todas las interacciones electromagnéticas, por lo tanto se reemplaza $e_{i} A_{\mu} \rightarrow e_{i 0} \epsilon A_{\mu}$. Sea $a_{\mu} \equiv \epsilon A_{\mu}$, entonces el tensor del campo electromagnético es,

$$
f_{\mu \nu} \equiv a_{\nu, \mu}-a_{\mu, \nu}
$$

y la acción electromagnética,

$$
S_{f}=-\frac{1}{16 \pi} \int e^{-2 \psi} f_{\mu \nu} f^{\mu \nu}(-g)^{\frac{1}{2}} d^{4} x .
$$

Las hipótesis 2 y 3 obligan a introducir una acción para $\epsilon$. Para no violar la hipótesis 5 , se excluyen los lagrangianos con curvatura. Por lo tanto, la única posibilidad para la acción libre de $\epsilon$ es,

$$
S_{\epsilon}=-\frac{c \hbar}{2 \ell_{B}^{2}} \int \epsilon^{-2} \epsilon_{, \mu} \epsilon^{\mu}(-g)^{\frac{1}{2}} d^{4} x,
$$

donde $g$ es el determinante de la métrica $g_{\mu \nu}, \ell_{B}$ es una constante de longitud introducida por razones dimensionales que no puede ser menor que la longitud de Planck.

La acción total es $S=S_{\epsilon}+S_{f}+S_{m}+S_{g}$, $\left(S_{m}\right.$ acción de la materia incluyendo la interacción electromagnética)

$$
S_{m}=\sum \int\left[-m c^{2}+\left(\frac{e_{0} \epsilon}{c}\right) u^{\mu} A_{\mu}\right] \times \gamma^{-1} \delta^{3}\left[x^{i}-x^{i}(\tau)\right] d^{4} x,
$$


$\mathrm{y}$

$$
S_{g}=\frac{c^{4}}{16 \pi G} \int R(-g)^{\frac{1}{2}} d^{4} x .
$$

Gracias a la variación de $\delta S_{m} / \delta x^{\mu}$ en combinación con $u_{\mu} u^{\mu}=-c^{2}$ se obtiene la expresión,

$$
d\left(m u_{\mu}\right) / d \tau=-m_{, \mu} c^{2}+\left(e_{0} / c\right)\left[\left(\epsilon A_{\nu}\right)_{, \mu}-\left(\epsilon A_{\mu}\right)_{, \nu}\right] u^{\nu} ;
$$

de la cual se desprende que, además de la fuerza tipo "Lorentz" (representada por el segundo miembro del lado derecho de la ecuación), aparece un término anómalo proveniente de la dependencia de la masa con el campo escalar $\epsilon$. Luego, variando $S_{\epsilon}+S_{f}+S_{m}$ respecto a $A_{\mu}$, se obtienen las ecuaciones para el campo electromagnético,

$$
\begin{gathered}
\left(\frac{1}{\epsilon} F^{\mu \nu}\right)_{; \nu}=4 \pi j^{\mu} \\
j^{\mu}=\sum\left[\frac{e_{0}}{c \gamma}\right] u^{\mu}(-g)^{-\frac{1}{2}} \delta^{3}\left[x^{i}-x^{i}(\tau)\right],
\end{gathered}
$$

cumpliéndose la condición $j_{; \mu}^{\mu}=0$. La carga conservada $\left(\sum e_{0}\right)$ de todas las partículas se distingue de la suma de las cargas $e_{0} \epsilon$ que se acoplan con $A_{\mu}$ en la acción.

Experimentalmente es sabido que $\epsilon$ varía muy poco en escalas de tiempo y espacio terrestres, por lo cual es posible sacar al factor $\epsilon^{-1}$ fuera de la derivada en la ecuación del campo electromagnético. Además, la escala de $\epsilon$ se elige de manera tal que en la época cosmológica actual, $\epsilon=1 \mathrm{y}$ muy lejos de la fuente del campo $\epsilon$. Con esta elección, $e_{0}$ coincide con la carga usual de partículas.

Las ecuaciones para $\epsilon$ se obtienen al derivar $S=S_{\epsilon}+S_{f}+S_{m}$ respecto de $\ln \epsilon$,

$$
\begin{gathered}
\square \ln \epsilon=\frac{\ell_{B}^{2}}{c \hbar}\left[\epsilon \frac{\partial \sigma}{\partial \epsilon}-\frac{1}{8 \pi} F^{\mu \nu} F_{\mu \nu}\right], \\
F_{\mu \nu}=\epsilon^{-1}\left[\left(\epsilon A_{\nu}\right)_{, \mu}-\left(\epsilon A_{\mu}\right)_{, \nu}\right], \\
\sigma=\sum m c^{2} \gamma^{-1}(-g)^{-\frac{1}{2}} \delta^{3}\left[x^{i}-x^{i}(\tau)\right],
\end{gathered}
$$

donde $\square$ es el D’Alambertiano. 


\subsection{El campo $\epsilon$ producido por una carga puntual en reposo}

Se toman partículas libres en reposo dentro de una cavidad conductora esférica. En este caso se trabaja en el espacio-tiempo plano, $F^{0 i}$ representa al vector del campo eléctrico $\boldsymbol{E}$, siendo entonces $\mu=0$ se obtiene,

$$
\vec{\nabla} \cdot\left(\epsilon^{-1} \boldsymbol{E}\right)=0
$$

El campo eléctrico en el interior de la cavidad es nulo, mientras que en el exterior,

$$
\boldsymbol{E}=\frac{\widehat{r} Q \epsilon}{r^{2}}, \quad Q \sim \sum e_{0}
$$

Reemplazando en la ecuación para $\epsilon$, se llega a que fuera de la cavidad,

$$
r^{-2} \partial_{r}\left[r^{2}\left(\partial_{r} \ln \epsilon\right)\right]=(4 \pi c \hbar)^{-1} \ell_{B}^{2} Q^{2} \epsilon^{2} r^{-4}, \quad F^{\mu \nu} F_{\mu \nu}=-2 \boldsymbol{E}^{2} .
$$

Transformando apropiadamente, el resultado es,

$$
\epsilon(r)=\sec \left[\ell_{B} Q(4 \pi \hbar c)^{-\frac{1}{2}} r^{-1}\right]
$$

Cuando $r \rightarrow \infty, \epsilon \rightarrow 1$. A grandes distancias el campo eléctrico tiene una forma puramente Coulombiana $\boldsymbol{E}=\widehat{r} Q r^{-2}$. Si hubiese un sola carga, el comportamiento coulombiano es preciso para $r \geq \ell_{B}$.

\subsection{Las variaciones temporales de $\alpha$}

Estas variaciones están relacionadas con los cambios en $\epsilon$ en el marco cosmológico. Se asume que el Universo es isotrópico y homogéneo. Adoptando la métrica de Robertson-Walker con el agregado del factor de expansión $a(t)$, la ecuación (2.11a) se reescribe,

$$
\frac{\partial}{\partial t}\left(a^{3} \frac{\dot{\epsilon}}{\epsilon}\right)=-\frac{a^{3} \ell_{B}^{2}}{c \hbar}\left[\epsilon \frac{\partial \sigma}{\partial \epsilon}+\frac{1}{4 \pi}\left(\boldsymbol{E}^{2}-\boldsymbol{B}^{2}\right)\right]
$$


$\boldsymbol{E}, \boldsymbol{B}$ son los campos medidos por un observador en el movimiento. Como aquí no interesan las variaciones espaciales de $\epsilon$ ( debido a que su muy pequeña variación en el contexto del Universo), el término fuente en el lado derecho de la útima ecuación se lo puede entender como un promedio en un gran volumen.

La radiación electromagnética del Universo no contribuye a la fuente de $\epsilon$, ya que para ondas planas, la invariancia $\boldsymbol{E}^{2}-\boldsymbol{B}^{2}$ desaparece, y una distribución incoherente es una superposición lineal de ellas con fases no definidas. Se concluye entonces que, la única fuente de $\epsilon$ es la materia.

Ambas partes de la ec.(2.16) son invariantes de Lorentz por lo tanto, se puede pasar a un sistema en reposo y evaluar allí la contribución de la partícula a la fuente. Promediando esta contribución en un gran volumen, el témino de la fuente queda,

$$
\left[\epsilon\left(\frac{\partial m}{\partial \epsilon}\right) c^{2}+(4 \pi)^{-1} \int_{V_{0}}\left(\boldsymbol{E}^{2}-\boldsymbol{B}^{2}\right) d V\right] V_{0}^{-1} .
$$

Reescribiendo en función de la parte electromagnética de la energía $m c^{2}$,

$$
\left[\epsilon\left(\frac{\partial m}{\partial \epsilon}\right) c^{2}+2 m_{E M} c^{2}-(2 \pi)^{-1} \int_{V_{0}} \boldsymbol{B}^{2} d V\right] V_{0}^{-1}
$$

El último término que está relacionado con el campo magnético se desprecia ya que la energía magnética es muy pequeña en la materia no relativista y; como gran parte de $m_{E M} c^{2}$ es de origen coulombiano, se tiene que $\epsilon\left(\frac{\partial m}{\partial \epsilon}\right) c^{2} \approx 2 m_{E M} c^{2}$.

Los principales contribuyentes de la densidad de energía coulombiana son los protones y neutrones (ya sean libres o ligados en núcleos) que conforman casi toda la materia en reposo del Universo. Las energías de los protones y neutrones están dadas por, $m_{E M p} c^{2} \approx 3 \mathrm{MeV}$ y $m_{E M n} c^{2} \approx 4,3 \mathrm{MeV}$. La mayoría de los neutrones del Universo han sido incorporados a núcleos de ${ }^{4} \mathrm{He}$ que constituyen un cuarto de la masa de la materia. Estos nucleones en ${ }^{4} \mathrm{He}$ contribuyen sólo un 15 por ciento más que los libres a la energía de Coulomb, en cambio cuando se encuentran en ${ }^{16} \mathrm{O}$ o ${ }^{56} \mathrm{Fe}$ lo hacen un 40 y un 70 por ciento más que cuando están libres. Debido a que los elementos más abundantes son el ${ }^{1} \mathrm{H}$ y ${ }^{4} \mathrm{He}$ (justamente son los más livianos), la energía coulombiana promedio que proviene de los nucleones excede solamente un 10 por ciento a la que proviene de protones libres. Entonces, siendo $\rho_{m}$ la densidad de 
masa de la materia en reposo,

$$
\frac{\partial}{\partial t}\left(a^{3} \frac{\dot{\epsilon}}{\epsilon}\right)=-a^{3} \zeta\left(\frac{\ell_{B}^{2}}{c \hbar}\right) \rho_{m} c^{2}, \quad \zeta \approx 1,3 \times 10^{-3}
$$

Si bien $\zeta$ depende de las propiedades del material que constituye a la fuente, se lo puede considerar constante ya que, para que sufra un cambio significativo, tendría que producirse una gran modificación en la abundancia relativa del $\mathrm{H}$ u otro tipo de elemento significativo. Dado que $\rho$ varía como $a^{-3}$ en un Universo en expansión,

$$
\frac{\dot{\epsilon}}{\epsilon}=-\zeta\left(\frac{\ell_{B}^{2} c}{\hbar}\right) \rho_{m}\left(t-t_{c}\right) \quad t_{c} c t e .
$$

Reescribiendo en notación cosmólogica,

$$
\frac{\dot{\epsilon}}{\epsilon}=-\frac{3 \zeta}{8 \pi}\left(\frac{\ell_{B}}{\ell_{P}}\right)^{2} H_{0}^{2} \Omega_{B}\left[\frac{a_{0}}{a(t)}\right]^{3}\left(t-t_{c}\right) .
$$

El Modelo Estándar de la Nucleosíntesis Primordial predice que la constante de integración $t_{c}$ debe ser muy pequeña para no perder el acuerdo entre la teoría y la observación. Usando los datos provenientes de WMAP (sonda de la NASA cuya misión es estudiar el cielo y medir las diferencias de temperatura y de polarización que se observan en la radiación de fondo de microondas, un remanente del Big Bang) se puede predecir que para $(\dot{\alpha} / \alpha)_{0}$ se cumple la siguiente ecuación,

$$
\left(\frac{\dot{\alpha}}{H_{0} \alpha}\right)_{0}=1,3 \times 10^{-5}\left(\frac{\ell_{B}}{\ell_{P}}\right)^{2} .
$$

Cualquier medida con una precisión tal que $\sigma\left(\dot{\alpha} / H_{0} \alpha\right) \sim 10^{-5}$ es difícil de lograr, por lo que la comparación entre la teoría y experimento no es una tarea fácil de llevar a cabo.

\subsection{Las variaciones espaciales de $\alpha$}

Debido a la presencia de materia con estructura electromagnética, se deberían poder encontrar gradientes de $\alpha$ en el laboratorio. A continuación se detalla la apli- 
cación del modelo para: 1) una partícula cargada en reposo, 2) para un sistema de partículas cargas, y 3) para un conjunto de dipolos magnéticos.

\subsubsection{Modelo para una carga puntual}

Tomando el caso de una carga puntual estática y reemplazando $\ln \epsilon=\psi$, las ecuaciones (2.10) y (2.11) pueden expresarse como,

$$
\begin{gathered}
\nabla \cdot\left(e^{-2 \psi} \boldsymbol{E}\right)=4 \pi e_{0} \delta^{3}(\boldsymbol{x}) \\
\nabla^{2} \psi=\frac{\ell_{B}^{2}}{c \hbar}\left[\frac{\partial m c^{2}}{\partial \psi} \delta^{3}(\boldsymbol{x})+\frac{1}{4 \pi} e^{-2 \psi} \boldsymbol{E}^{2}\right]
\end{gathered}
$$

donde,

$$
\begin{gathered}
\boldsymbol{E}=e^{2 \psi}(-\nabla \Phi+\boldsymbol{b}), \\
\nabla . \boldsymbol{b}=0, \\
\Phi=e_{0} / r .
\end{gathered}
$$

$\Phi$ representa al potencial Coulombiano, y se toma una solución particular tal que $\boldsymbol{b}$ sea un vector constante. Por lo tanto, se puede considerar al campo $\psi(\boldsymbol{r})$ como función del potencial Coulombiano, $\psi[\Phi(\boldsymbol{r})]$. Luego,

$$
\begin{aligned}
\nabla^{2} \psi & =\frac{\partial \psi}{\partial \Phi} \nabla^{2} \Phi+\frac{\partial^{2} \psi}{\partial \Phi^{2}} e^{-4 \psi} \boldsymbol{E}^{2} \\
& =-4 \pi \rho \frac{\partial \psi}{\partial \Phi}+\psi^{\prime \prime} e^{-4 \psi} \boldsymbol{E}^{2}
\end{aligned}
$$

Para que las ecuaciones (2.23b) y (2.25) sean comparables, es necesario que se cumplan las siguientes condiciones,

$$
\psi^{\prime \prime}=\kappa^{2} e^{2 \psi}, \quad \kappa^{2} \frac{\partial \sigma}{\partial \psi}=-e_{0} \frac{\partial \psi}{\partial \Phi}
$$

de manera de tener una descripción de $\sigma$ y de $\psi$ en función de $\Phi$ tal que, $\frac{\partial m c^{2}}{\partial \psi} \delta^{3}(x)+\frac{1}{4 \pi} e^{-2 \psi} \boldsymbol{E}^{2}=0$ en el Laplaciano de $\psi$. 


\subsubsection{Modelo para un sistema de cargas}

Para el caso de un conjunto de cargas en reposo (un sistema de $N$ cargas, cada una con posición $\left.z_{i}\right), e_{0} \delta^{3}(\boldsymbol{x})$ se reemplaza por $\sum_{i} e_{0 i} \delta^{3}\left(\boldsymbol{x}-\boldsymbol{z}_{\boldsymbol{i}}\right)$, y el potencial será,

$$
\Phi(\boldsymbol{r})=\sum_{i} \frac{e_{0 i}}{\left|\boldsymbol{x}-\boldsymbol{z}_{\boldsymbol{i}}\right|} .
$$

Haciendo un análisis similar al hecho en el caso de una carga puntual se llega a que,

$$
e^{\psi}=\sec (\kappa \Phi), \quad \boldsymbol{E}=-\nabla \frac{\tan (\kappa \Phi)}{\kappa},
$$

cancelándose el corchete de la ec.(2.23b).

La idea del modelo es la siguiente; se propone un cúmulo de partículas que se encuentra en el origen actuando como si fuera "gran partícula" $C$ y se toma otra partícula a una distancia mucho mayor a las dimensiones del cúmulo. La partícula $C$ estaría inmersa en los campos uniformes producidos por la otra partícula, y se pide además que a $t=0$ todas se encuentren en reposo. Trabajando con las partículas que conforman al "grupo" $C$, se llega a que en este caso, la estructura interna del cúmulo no afecta la tasa de cambio del momento de la "partícula" total. Se estudia luego el sistema en su totalidad, analizando la componente temporal de la conservación del tensor de energía impulso. Si $T^{\mu \nu}$ incluye al tensor para las partículas, al del electromagnetismo y al del los campos escalares, $T_{\nu}^{\mu \nu}=0$ se puede derivar de las ecuaciones del campo gravitacional. Ignorando a este último campo mencionado,

$$
\frac{\partial T^{00}}{\partial t}+\frac{\partial T^{0 i}}{\partial x^{i}}=0
$$

multiplicando por $x^{j}$ e integrando en un volumen que contenga a $C$ pero no a la otra partícula,

$$
\frac{d}{d t} \int_{\mho} T^{00} x^{j} d^{3} x=\int_{\mho} T^{0 j} d^{3} x-\oint_{\partial \mho} x^{j} T^{0 i} d^{2} S_{i},
$$

donde $M=\int_{\mho} T^{00} d^{3} x$ es la masa del cúmulo despreciando las contribuciones de los 
campos más allá de las cercanías de $C$. Luego,

$$
\frac{d^{2}}{d^{2} t} \int_{\mho} T^{00} x^{j} d^{3} x=\frac{d}{d t} \int_{\mho} T^{0 j} d^{3} x-\frac{d}{d t} \oint_{\partial \mho} x^{j} T^{0 i} d^{2} S_{i}
$$

$T^{0 i}$ tiene contribuciones de:

- El campo $f^{\mu \nu}$, pero debido a se está trabajando en el caso estático para el set de ecuaciones (2.23), y siendo la identidad $f_{i j}+f_{0 i, j}+f_{j 0, i}=0$; la derivada temporal elimina la integral de esta contribución $\left(T_{f}^{0 i}\right)$ a $t=0$.

- El campo $\psi$ a través de $T_{\psi}^{0 i}$, pero también la derivada temporal hace desaparecer su integral a $t=0$ ya que no hay movimiento. Mirando ecuaciones anteriores (2.23) y sus soluciones (2.28) para el reposo, se deduce que $\dot{\psi}$ y $\ddot{\psi}$ son nulas a $t=0$.

- Por lo tanto, sólo las partículas contribuyen a dicha parte de la ecuación. Dado que la superficie $\partial \mho$ se desvanece más allá de $C$, el lado izquierdo de la ecuación (2.31) se reduce a $\frac{d}{d t} \int_{\mho} T_{m}^{0 j} d^{3} x$. El tensor se obtiene de la acción $S_{m}$ tal que,

$$
T_{m}^{\mu \nu}=\sum_{i} m_{i} c \frac{d z_{i}^{\mu}}{d \tau} \frac{d z_{i}^{\nu}}{d \tau} \frac{\delta^{3}\left[x-z_{i}(\tau)\right]}{\gamma(-g)^{-1 / 2}} .
$$

Tomando $\tau=t$, reemplazando $(-g)^{-1 / 2}$ por su valor Minkowskiano y sustituyendo en (2.31),

$$
\frac{d^{2}\left(M Z^{j}\right)}{d t^{2}}=\frac{d}{d t} \sum_{i=2}^{N} m_{i} \boldsymbol{v}_{\boldsymbol{i}}
$$

Integrando la ec. (2.29) en el volumen, usando el teorema de Gauss y derivando respecto al tiempo,

$$
\frac{d^{2}}{d t^{2}} \int_{\mho} T^{00} d^{3} x=-\frac{d}{d t} \oint_{\partial \mho} T^{0 i} d^{2} S_{i}
$$

donde el lado derecho de la ecuación desaparece a $t=0$ por los mismos argumentos que en las ecuaciones anteriores. Por lo tanto, se demuestra que $\dot{M}=\ddot{M}=0$ a $t=0$, dándose que $M(t=0)+O\left(t^{3}\right)$ en un período corto de tiempo. Haciendo el cambio en las ecuaciones anteriores a $t=0$ se llega a que

$$
\ddot{\boldsymbol{Z}}=\frac{Q e_{01}\left(\boldsymbol{Z}-\boldsymbol{z}_{\mathbf{1}}\right)}{M\left|\boldsymbol{Z}-\boldsymbol{z}_{\mathbf{1}}\right|^{3}}
$$


siendo $\boldsymbol{Z}$ la posición del centro de masas de $C$. Esto en concordancia al WEP, muestra que la aceleración del CM del cúmulo en un campo distante de la carga $e_{01}$ está completamente determinado por su masa $M$ y su carga total $Q$. Para esto, la integral de $T^{00}$ debe ser hecha sobre una región finita. Todos estos resultados son ciertos siempre y cuando las partículas del cúmulo sean estáticas, pero se supone que a bajas velocidades y espines magnéticos débiles también se cumplen.

\subsubsection{Modelo para una conjunto de dipolos magnéticos}

En la materia bariónica, la segunda contribución energética más grande luego de la Coulombiana es aquella que proviene de los dipolos magnéticos asociados con el momento angular y espines de los núcleos y electrones. La energía magnética ha sido hasta ahora ignorada en este modelo. Dado que se ha demostrado que los aportes Coulombianos son poco menos que irrelevantes como fuentes del campos $\psi$, es necesario tener en cuenta las contribuciones magnéticas.

Es muy difícil encontrar directamente el análogo al sistema de cargas cuya solución está dada por las expresiones de la ecuación ( 2.28) para una colección de dipolos magnéticos. Para superar el problema se propone usar un conjunto de monopolos magnéticos de "carga magnética" $g_{0 i}, i=1,2, \ldots 2 N$, y luego dejar que los pares de igual módulo y opuesta carga de monopolo se fusionen para formar los dipolos magnéticos. Estos los monopolos sólo servirán como "ayuda" en el cálculo y desaparecerán en los resultados finales. Las ecuaciones a resolver son,

$$
\begin{gathered}
\nabla \times\left(e^{-2 \psi} \boldsymbol{B}\right)=0 \\
\nabla \cdot \boldsymbol{B}=\sum_{i} 4 \pi g_{0 i} \delta^{3}\left(\boldsymbol{x}-\boldsymbol{z}_{\boldsymbol{i}}\right), \\
\nabla^{2} \psi=\frac{\ell_{B}^{2}}{c \hbar}\left[\sum_{i} \frac{\partial m_{i} c^{2}}{\partial \psi} \delta^{3}\left(\boldsymbol{x}-\boldsymbol{z}_{\boldsymbol{i}}\right)+\frac{1}{4 \pi} e^{-2 \psi} \boldsymbol{B}^{2}\right] .
\end{gathered}
$$

La ecuación (2.36b) no proviene de la acción para el campo $f\left(S_{f}\right)$ del modelo, sino que es más bien una versión generaliza la ley de Gauss $\nabla \cdot \boldsymbol{B}=0$ para el caso en que pudieran existir los monopolos. Este es el argumento para no incluir al factor $e^{-2 \psi}$ 
en dicha expresión. Por la forma que presenta la ecuación (2.36b), no es posible tener un potencial vector para $\boldsymbol{B}$ pero sí se lo puede describir, en un dominio, mediante un potencial escalar tal que,

$$
\begin{gathered}
\boldsymbol{B}=-\nabla \Psi \\
\Psi(\boldsymbol{r})=\sum_{i} \frac{g_{0 i}}{\left|\boldsymbol{x}-\boldsymbol{z}_{\boldsymbol{i}}\right|} .
\end{gathered}
$$

La ecuación (2.36a) implica que $\psi$ es una función de $\Psi, \psi(\Psi(\boldsymbol{r}))$. Resolviendo de manera similar al caso del sistema de partículas cargadas, se llega a una única solución física para un sistema de monopolos magnéticos,

$$
e^{\psi}=\cos (\kappa \Psi), \quad \boldsymbol{B}=-\nabla \Psi .
$$

Se toma un monopolo $i+N$ cuya carga magnética es $-g_{0 i}$, y otro monopolo $i$ tal que el vector posición entre ellos es $\boldsymbol{d}$. Luego, si el par se puede aproximar adiabáticamente, $\left|g_{0 i}\right|$ crece de modo que $g_{0 i} \boldsymbol{d} \rightarrow \boldsymbol{\mu} \neq 0$. Entonces en el límite se tiene un dipolo cuyo momento magnético es $\boldsymbol{\mu}$ y se lo puede "etiquetar" como el dipolo $i$, con posición $\boldsymbol{z}_{i}$. El potencial magnético total y el campo son,

$$
\begin{gathered}
\Psi=\lim _{|d| \rightarrow 0} \sum_{i=1}^{N}\left[\frac{g_{0 i}}{\left|\boldsymbol{x}-\boldsymbol{z}_{i}-\boldsymbol{d} / 2\right|}+\frac{-g_{0 i}}{\left|\boldsymbol{x}-\boldsymbol{z}_{\boldsymbol{i}}+\boldsymbol{d} / 2\right|}\right], \\
\Psi=\sum_{i=1}^{N} \frac{\boldsymbol{\mu} \cdot\left(\boldsymbol{x}-\boldsymbol{z}_{i}\right)}{\left|\boldsymbol{x}-\boldsymbol{z}_{\boldsymbol{i}}\right|^{3}} \\
\boldsymbol{B}=\sum_{i=1}^{N}\left[\frac{3 \boldsymbol{\mu} \cdot\left(\boldsymbol{x}-\boldsymbol{z}_{\boldsymbol{i}}\right)\left(\boldsymbol{x}-\boldsymbol{z}_{\boldsymbol{i}}\right)}{\left|\boldsymbol{x}-\boldsymbol{z}_{\boldsymbol{i}}\right|^{5}}-\frac{\boldsymbol{\mu}}{\left|\boldsymbol{x}-\boldsymbol{z}_{\boldsymbol{i}}\right|^{3}}\right] .
\end{gathered}
$$

Parece razonable que los dipolos magnéticos pueden ser representados por pequeños "bucles de corriente", y que el campo seguirá siendo el de la Ec. (2.40c). Las ecuaciones (2.39) y (2.40) representan las soluciones para un sistema de multidipolos magnéticos dentro este marco de trabajo. De la ecuación (2.39) se deriva,

$$
\nabla^{2} \psi=\kappa \tan (\kappa \Psi) \boldsymbol{\nabla} \cdot \boldsymbol{B}-\kappa^{2} \sec ^{2}(\kappa \Psi) \boldsymbol{B}^{2} .
$$

Aplicando el teorema de Gauss a la ec.(2.40c), $\boldsymbol{\nabla} \cdot \boldsymbol{B}=0$ en todas partes, y no 
hay singularidades del tipo $\delta$ en la posición de un dipolo. Además comparando la expresión (2.36c) con $e^{-2 \psi}=\sec ^{2}(\kappa \Psi)$ se concluye que el término fuente de masa no tiene una contribución de los dipolos magnéticos. Una consecuencia inmediata es que un el momento de un dipolo magnético en un campo electromagnético exterior no está sujeto a una fuerza anómala como la que aparece en el caso de la carga eléctrica. La única fuerza sería la habitual $(\boldsymbol{\mu} \cdot \boldsymbol{\nabla}) \boldsymbol{B}$, que se puede obtener en este contexto, mediante la combinación de las fuerzas en dos polos magnéticos. Todo esto significa que no se esperan violaciones del WEP en el movimiento de una estructura pura de dipolos magnéticos.

Las posibles violaciones del WEP que pueden llegar a aparecer en una partícula de prueba debido a la interacción con una fuente $S$ formada puramente por dipolos magnéticos, quedan fuera del alcance actual de los experimentos de laboratorio.

\subsubsection{Conclusiones}

La ecuación del campo $\psi$ para una colección de cargas y de dipolos magnéticos está dada por,

$$
\nabla^{2} \psi=\frac{\ell_{B}^{2}}{c \hbar}\left[\sum_{i} \frac{\partial m_{i} c^{2}}{\partial \psi} \delta^{3}\left(\boldsymbol{x}-\boldsymbol{z}_{\boldsymbol{i}}\right)+\frac{1}{4 \pi} e^{-2 \psi}\left(\boldsymbol{E}^{2}-\boldsymbol{B}^{2}\right)\right] .
$$

La suma se extiende sólo sobre las cargas eléctricas. Claramente, las contribuciones eléctricas y magnéticas al campo $\psi$ se suman.

Dado que $\sum_{i} \frac{\partial m_{i} c^{2}}{\partial \psi} \delta^{3}\left(\boldsymbol{x}-\boldsymbol{z}_{\boldsymbol{i}}\right)+\frac{1}{4 \pi} e^{-2 \psi} \boldsymbol{E}^{2} \approx 0$, última la ecuación queda,

$$
\nabla^{2} \psi=-\frac{\ell_{B}^{2}}{c \hbar}\left[\frac{1}{4 \pi} e^{-2 \psi} \boldsymbol{B}^{2}\right]
$$

y no hay evidencia experimetal de violación al Principio de Equivalencia. 


\section{Capítulo 3}

\section{El modelo de Bekenstein para el caso semiclásico}

En sus trabajos (Refs.[15, 16]), Bekenstein utiliza un conjunto de partículas clásicas para representar a la materia. Esto no es un buen modelo de materia cuando los fenómenos cuánticos pueden llegar a ser importantes, ya sea a escalas de altas energías y/o a escalas de distancias pequeñas; debido a que los fermiones tienen una "escala de longitud natural", es decir, la longitud de onda Compton de la partícula $\lambda_{C}=\hbar / m c$.

Por esto mismo, nosotros elegimos trabajar con un sistema más realista, un tratamiento semiclásico (como la teoría semiclásica de la radiación) en donde los campos electromagnético, newtoniano y dilatónico $\psi$ son tomados en forma clásica pero la materia es tratada en forma cuántica respondiendo a un modelo tipo ThomasFermi $[84,85]$. Además, supondremos que el campo escalar $\psi$ es pequeño. Agregando estas nuevas aproximaciones al modelo, analizamos los comportamientos de los campos eléctricos, magnéticos, y el escalar $\psi$; y de la distribución de carga.

\subsection{Desarrollo del modelo}

Consideramos en este caso un átomo como un sistema de partículas cuánticas, tal que aplicando la segunda cuantificación el hamiltoniano estará dado por: 


$$
\begin{gathered}
H=m c^{2}+H_{1}+H_{2}+H_{3}+H_{4}+H_{5}, \\
H_{\beta}=H_{1}+H_{2}+H_{5}, \\
H_{1}=\int \widehat{\Psi}^{\dagger}(\bar{\xi})\left(\frac{-\hbar^{2} \nabla^{2}}{2 m}\right) \widehat{\Psi}(\bar{\xi}) d \bar{\xi} \\
H_{2}=\int \widehat{\Psi}^{\dagger}(\bar{\xi}) e_{0} e^{\psi} \Phi(\bar{\xi}) \widehat{\Psi}(\bar{\xi}) d \bar{\xi} \\
H_{3}=\int \frac{(\nabla \Phi(\bar{\xi}))^{2}}{2} e^{-2 \psi} d \bar{\xi} \\
H_{4}=\int \frac{(\nabla \psi)^{2} \hbar c}{2 l^{2}} d \bar{\xi} \\
H_{5}=\int m_{0} \widehat{\Psi}^{\dagger}(\bar{\xi}) \phi(\bar{\xi}) \widehat{\Psi}(\bar{\xi}) d \bar{\xi}
\end{gathered}
$$

siendo $\Phi$ y $\phi$ los potenciales electrostático y gravitatorio respectivamente, y $\psi$ el campo escalar de Bekenstein.

En el límite no relativista el hamiltoniano no depende de los spines, por lo tanto $\bar{\xi}(\bar{r}, \sigma)$ se reduce a $\bar{\xi}(\bar{r})$, y además se cumple la ecuación de Schrödinger $i \hbar \frac{\partial \Psi}{\partial t}=H \Psi$.

Los operadores del campo de fermiones (ej. electrones) son,

$$
\widehat{\Psi}^{\dagger}(\bar{\xi})=\sum_{i} \Psi_{i}^{\dagger}(\bar{\xi}) \widehat{a}_{i}^{\dagger}, \quad \widehat{\Psi}(\bar{\xi})=\sum_{i} \Psi_{i}(\bar{\xi}) \widehat{a}_{i}
$$

donde la función de onda de un sistema de $n$ partículas que describe a los observables es,

$$
\boldsymbol{\Psi}_{\mathbf{n}}=\operatorname{det}\left(\begin{array}{ccc}
\Psi_{r_{1}}\left(\xi_{1}\right) & \ldots & \Psi_{r_{1}}\left(\xi_{n}\right) \\
\vdots & \ddots & \\
\Psi_{r_{n}}\left(\xi_{1}\right) & \ldots & \Psi_{r_{n}}\left(\xi_{n}\right)
\end{array}\right)
$$

Debido a las reglas de conmutación y anticonmutación de los operadores de aniquilación y creación $\widehat{a}_{i}$ y $\widehat{a}_{i}^{\dagger}$,

$$
\left[\widehat{a}_{i}, \widehat{a}_{j} \widehat{a}_{l}\right]=\delta_{i j} \widehat{a}_{l}, \quad\left[\widehat{a}_{i}, \widehat{a}_{j}^{\dagger} \widehat{a}_{k}^{\dagger} \widehat{a}_{l} \widehat{a}_{m}\right]=\delta_{i j} \widehat{a}_{k}^{\dagger} \widehat{a}_{l} \widehat{a}_{m}
$$

los operadores $\widehat{\Psi}(\xi)$ cumplen ciertas propiedades de clausura tal que, dado que el 
hamiltoniano $H_{\beta}$ es independiente del tiempo,

$$
i \hbar \frac{d \widehat{\Psi}}{d t}=\left[\widehat{\Psi}, \widehat{H}_{\beta}\right]=\sum_{i} \Psi_{i}(\xi)\left[\widehat{a}_{i}, \widehat{H}_{\beta}\right]
$$

Luego, es posible reemplazar los operadores en el $H_{\beta}$ y resolver utilizando dichas propiedades,

$$
\sum_{i} \Psi_{i}(\xi)\left[\widehat{a}_{i}, \widehat{H}_{\beta}\right]=\Theta_{1}+\Theta_{2}+\Theta_{3}
$$

Dado,

$$
\begin{gathered}
\Theta_{1}=\sum_{i, l} \Psi_{i}(\xi) h_{j}^{(1) i} \widehat{a}_{l}, \\
h_{j}^{(1) i}=\int \Psi_{i}^{*}(\xi) h^{(1)} \Psi_{i}(\xi) d \xi \\
h^{(1)}=-\frac{\hbar \nabla^{2}}{2 m}
\end{gathered}
$$

donde los elementos de la diagonal de $h_{j}^{(1) i}$ representan los valores de expectación de $h^{(1)}$ en los estados $\Psi_{i}(\xi)$; mientras que los elementos fuera de la diagonal $(i \neq j)$ representan las transiciones entre los estados $j$ e $i$ los cuales son inducidas por $h^{(1)}$. Mientras que,

$$
\begin{gathered}
\Theta_{2}=e_{0} e^{\psi} \sum_{i, j, l, m} \Psi_{i}(\xi) \Phi_{l m}^{(2) i j} \widehat{a}_{j}^{\dagger} \widehat{a}_{l} \widehat{a}_{m}, \\
\Phi_{l m}^{(2) i j}=\iint \Psi_{i}^{*}(\xi) \Psi_{j}^{*}\left(\xi_{2}\right) \Phi^{(2)} \Psi_{l}\left(\xi_{1}\right) \Psi_{m}\left(\xi_{2}\right) d \xi_{1} d \xi_{2},
\end{gathered}
$$

$\mathrm{y}$

$$
\begin{gathered}
\Theta_{3}=m_{0} \sum_{i, j, l, k} \Psi_{i}(\xi) \phi_{l m}^{(2) i j} \widehat{a}_{j}^{\dagger} \widehat{a}_{l} \widehat{a}_{m}, \\
\phi_{l m}^{(2) i j}=\iint \Psi_{i}^{*}(\xi) \Psi_{j}^{*}\left(\xi_{2}\right) \phi^{(2)} \Psi_{l}\left(\xi_{1}\right) \Psi_{m}\left(\xi_{2}\right) d \xi_{1} d \xi_{2},
\end{gathered}
$$

$\Phi_{l m}^{(2) i j}$ y $\phi_{l m}^{(2) i j}$ son las energías potenciales de interacción electrostática y gravitatoria 
entre las partículas $l$ y $m$. Resumiendo,

$$
\begin{gathered}
\Theta_{1}=\int\left(\sum_{i, l} \Psi_{i}^{*}\left(\xi^{\prime}\right) h_{l}^{(1) i} \Psi_{i}(\xi)\right) \widehat{\Psi}\left(\xi^{\prime}\right) d \xi^{\prime}=h^{(1)}(\xi) \widehat{\Psi}(\xi), \\
\Theta_{2}=e_{0} e^{\psi}\left(\int \Phi^{(2)}\left(\xi, \xi^{\prime}\right) \Psi^{\dagger}\left(\xi^{\prime}\right) d \xi^{\prime}\right) \widehat{\Psi}(\vec{\xi}), \\
\Theta_{3}=m_{0}\left(\int \phi^{(2)}\left(\xi, \xi^{\prime}\right) \Psi^{\dagger}\left(\xi^{\prime}\right) \Psi\left(\xi^{\prime}\right) d \xi^{\prime}\right) \widehat{\Psi}(\vec{\xi}) .
\end{gathered}
$$

Por lo tanto, la evolución del operador queda,

$$
i \hbar \frac{d \widehat{\Psi}}{d t}=\left[\widehat{\Psi}, \widehat{H}_{\beta}\right]=\left(h^{(1)}+\Phi_{e f}(\xi)+\phi_{e f}(\xi)\right) \widehat{\Psi},
$$

siendo

$$
\begin{gathered}
\Phi_{e f}(\xi)=\int \Phi^{(2)}\left(\xi, \xi^{\prime}\right) \Psi^{\dagger}\left(\xi^{\prime}\right) \Psi\left(\xi^{\prime}\right) d \xi^{\prime} e e^{-2 \psi}, \\
\phi_{e f}(\xi)=\int \phi^{(2)}\left(\xi, \xi^{\prime}\right) \Psi^{\dagger}\left(\xi^{\prime}\right) \Psi\left(\xi^{\prime}\right) d \xi^{\prime} m_{0} .
\end{gathered}
$$

Tanto $\Phi_{e f}$ como $\phi_{e f}$ describen las interacciones eléctricas y gravitatorias entre las partículas. Los potenciales efectivos sólo se puede calcular si la solución de la ecuación (3.10) es ya conocida. Esto sugiere el uso de métodos interactivos a fin de encontrar soluciones autoconsistentes la ecuación de movimiento, por ejemplo, los métodos que se utilizan para resolver los problemas de muchos cuerpos. La densidad del número de partículas se escribe como,

$$
\eta\left(\xi^{\prime}\right)=\Psi^{\dagger}\left(\xi^{\prime}\right) \Psi\left(\xi^{\prime}\right)
$$

Retomando entonces el hamiltoniano inicial de la ecuación (3.1a),

$$
H=\sum_{i} m_{i} c^{2}+\sum_{i} \frac{p_{i}^{2}}{2 m_{i}}+\theta_{1}+\theta_{2}+\theta_{3}+\theta_{4}
$$


con

$$
\begin{gathered}
\theta_{1}=\int e_{0} e^{\psi} \eta\left(r^{\prime}\right) \Phi\left(r^{\prime}\right) 4 \pi r^{\prime 2} d r^{\prime} \\
\theta_{2}=\int m_{0} \phi(r) \eta\left(r^{\prime}\right) 4 \pi r^{\prime 2} d r^{\prime} \\
\theta_{3}=\int \frac{\left(\nabla \Phi\left(r^{\prime}\right)\right)^{2}}{2} e^{-2 \psi} 4 \pi r^{\prime 2} d r^{\prime} \\
\theta_{4}=\int \frac{c \hbar}{l^{2}}(\nabla \psi)^{2} 4 \pi r^{\prime 2} d r^{\prime}
\end{gathered}
$$

\subsubsection{Modelo de Thomas-Fermi cuántico}

Este modelo basado en la mecánica estadística, fue desarrollado por Llewellyn Thomas y Enrico Fermi en 1927 [52, 133] para intentar explicar la estructura electrónica de sistemas con varias partículas (el detalle del tratamiento semiclásico se encuentra en el apéndice A). Su desarrollo puede ser extendido en forma cuántica utilizando la Teoría de Densidad Funcional (DFT), que es un método cuántico para investigar la estructura electrónica, principalmente el estado fundamental, de sistemas de muchos cuerpos como núcleos, atómos, moléculas, etc. (para más detalles ver $[7,21]$ entre otros). Presentaremos un breve resumen de esta teoría.

\section{Teoría de Densidad Funcional}

Ella describe propiedades de los sistemas multielectrónicos como átomos y núcleos mediante la utilización de funcionales, que dependen espacialmente de la densidad electrónica. Si bien los resultados de este tipo desarrollo para sistemas de estado sólido concuerdan muy satisfactoriamente con los datos experimentales y el costo computacional es relativamente bajo en comparación con los métodos tradicionales (Hartree-Fock y sus descendientes); existen dificultades en el uso de la teoría para describir correctamente las interacciones intermoleculares. Su origen se encuentra en el modelo de Thomas-Fermi pero su base teórica está dada por dos teoremas cuánticos de Hohenberg-Kohn (HK) [77], los cuales originalmente sólo tenían en cuenta los estados fundamentales no degenerados en la ausencia de campos magnéticos, pero luego fueron generalizados para abarcarlos. Esencialemente, estas proposiciones demuestran que; 
- para el estado fundamental, existe una relación unívoca entre la densidad y la función de onda que representa a los observables tal que, entre ellas contienen la misma información, y toda propiedad observable de un sistema puede calcularse a partir de la densidad electrónica;

- además, la energía es una funcional de la densidad y la densidad del sistema minimiza a esta funcional.

La función de onda para $\mathrm{N}$ partículas depende de $3 \mathrm{~N}$ variables, mientras que la densidad es más fácil de manejar ya que es siempre una función de sólo 3 variables, independientemente del número de partículas. Así, el problema de muchos cuerpos de electrones que interactúan en un potencial externo estático se reduce a un problema de electrones que no interactúan moviéndose en un potencial efectivo que incluye al potencial externo y los efectos de las interacciones de Coulomb entre los electrones, por ejemplo, el intercambio y la correlación entre ellos. El modelado de las dos últimas interacciones es bastante complicado. La aproximación más simple es la Aproximación Local de la Densidad (LDA), consiste en suponer que en cada punto, la energía de intercambio y correlación de un gas de electrones uniforme, que se puede obtener a partir del modelo de Thomas-Fermi, depende sólo de la densidad en ese punto. Los sistemas no interactuantes son relativamente fáciles de resolver dado que la función de onda se puede representar como un determinante de Slater de los orbitales. A pesar de tener una funcional total de energía desconocida que debe ser aproximada, la energía cinética funcional de este sistema se conoce con exactitud.

La ecuación de Schrödinger para un estado estacionario electrónico descripto por un función de onda $\Psi\left(\overrightarrow{r_{1}}, \ldots, \overrightarrow{r_{N}}\right)$ donde el potencial externo estático $V$ en el cual se mueven los electrones es generado por un núcleo fijo (aproximación de BornOppenheimer), está dado por;

$$
\hat{H} \Psi=[\hat{T}+\hat{V}+\hat{U}] \Psi=E \Psi
$$

siendo $\hat{H}$ el Hamiltoniano de un sistema de $\mathrm{N}$ electrones y $E$ la energía total tal que, $\hat{T}$ representa a la energía cinética, $\hat{V}$ es la energía potencial externa generada por el núcleo positivo, y $\hat{U}$ la energía de interacción electrón-electrón. No es posible separar esta ecuación de varios electrones en otras más simples de una sola partícula debido 
al término de interacción $\hat{U}$; pero la DFT proporciona una alternativa por la cual se mapea el problema de muchos cuerpos con $\hat{U}$, a un problema de solo cuerpo sin el término de interacción. La variable clave es la densidad de partículas $n(\vec{r})$,

$$
n(\vec{r})=N \int d^{3} r_{2} \int d^{3} r_{3} \ldots \int d^{3} r_{N} \Psi^{\dagger}\left(\vec{r}, \overrightarrow{r_{2}}, \ldots, \vec{r}_{N}\right) \Psi\left(\vec{r}, \overrightarrow{r_{2}} \ldots, \vec{r}_{N}\right),
$$

$\Psi$ normalizada. Siendo $n_{0}$ la densidad de partículas en el estado fundamental, $\Psi_{0}=$ $\Psi\left[n_{0}\right]$ por estar unívocamente determinada. Luego, la energía total del estado fundamental también es una funcional de $n_{0}$ (para un estado $n$ será una funcional $E[n]$ ),

$$
\begin{gathered}
E_{0}=E\left[n_{0}\right]=<\Psi\left[n_{0}\right]|\hat{T}+\hat{V}+\hat{U}| \Psi\left[n_{0}\right]>, \\
\hat{V}_{0}=<\Psi_{0}|\hat{V}| \Psi_{0}>=\hat{V}\left[n_{0}\right]=\int V(\vec{r}) n_{0}(\vec{r}) d^{3} r .
\end{gathered}
$$

Las funcionales $\hat{T}[n]$ y $\hat{U}[n]$ son "universales", mientras que $\hat{V}[n]$ depende del sistema en estudio. Mediante el método de multiplicadores de Lagrange se puede resolver el problema variacional para minimizar la funcional de energía total. Primero se considera un sistema donde no aparece explícitamente el término de interacción entre electrones tal que,

$$
E_{s}[n]=<\Psi[n]\left|\hat{T}+\hat{V}_{s}\right| \Psi[n]>
$$

$\hat{T}$ sigue describiendo la energía cinética y $\hat{V}_{S}$ es la energía de un potencial externo

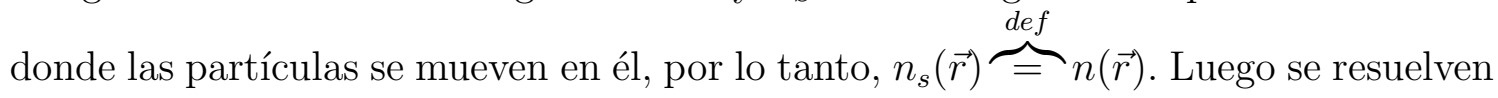
las ecuaciones para el sistema auxiliar no interactuante de Kohn-Sham,

$$
\left[-\frac{\hbar^{2}}{2 m} \nabla^{2}+V_{s}(\vec{r})\right] \chi_{i}(\vec{r})=\varepsilon_{i} \chi_{i}(\vec{r})
$$

siendo $\chi_{i}$ los orbitales $\mathrm{y}$,

$$
n_{s}(\vec{r})=\sum_{i}^{N}\left|\chi_{i}(\vec{r})\right|^{2}
$$


El potencial efectivo para una partícula estudiado con mayor detalle está dado por,

$$
V_{s}(\vec{r})=V(\vec{r})+\int \frac{e^{2} n_{s}\left(\overrightarrow{r^{\prime}}\right)}{\left|\vec{r}-\overrightarrow{r^{\prime}}\right|} d^{3} r^{\prime}+V_{X C}\left[n_{s}(\vec{r})\right]
$$

donde el segundo término del lado derecho de la expresión representa la repulsión coulombiana entre electrones, mientras que el último término es el potencial de intercambio y correlación que incluye todas las interacciones de varias partículas. Dado que estos dos también dependen de $n_{s}(\vec{r}), V_{s}$ debe resolverse manera iterativa; empezando por un valor de $n(\vec{r})$ estimativo, calculando el $V_{s}$ correspondiente y resolviendo las ecuaciones (3.19) para obtener los $\chi_{i}$, y luego recalcular el $n(\vec{r})$ para volver a resolver las ecuaciones; así sucesivamente hasta alcanzar la convergencia. También existe un método que no es iterativo llamado el Funcional de Harris [63].

Luego es posible definir las densidades de carga y masa de un sistema como,

$$
\rho_{c}(\vec{r})=Q n(\vec{r}) \quad \rho_{m}(\vec{r})=m n(\vec{r})
$$

siendo $Q$ y $m$ la carga y la masa de las partículas que lo conforman.

La teoría expuesta muestra que en un sistema cuántico, el acoplamiento con el campo electromagnético puede tratarse introducinendo densidades de carga y de corriente suaves.

\subsection{Movimiento de un cuerpo en el campo $\psi$}

En esta parte del capítulo estudiamos el movimiento de distribuciones de materia en el campo escalar $\psi$, y las posibles contribuciones de los campos eléctrico y magnético. Analizamos el Lagrangiano de un cuerpo compuesto por cargas puntuales tales como, por ejemplo un átomo o un núcleo atómico. Trabajamos en el límite no relativista para las cargas, pero manteniendo por el momento la plena expresión para el campo electromagnético. Solamente tendremos en cuenta los campos dilatónico externo $\psi$ y gravitatorio $\phi_{N}$ que actúan sobre el cuerpo, despreciando así los autogenerados. Los lagrangianos de materia, campos electromagnético y es- 
calar $\epsilon$ para $c=1$ y siguiendo la notación de Bekenstein ${ }^{1}$, toman las siguientes formas,

$$
\begin{gathered}
L_{m}=\int\left[\bar{\Psi}\left(i \partial_{\mu}-m+e_{0} \epsilon \gamma^{\mu} A_{\mu}\right) \Psi\right] d^{3} x, \\
L_{E M}=\frac{-1}{16 \pi} \int F_{\mu \nu} F^{\mu \nu} \sqrt{-g} d^{3} x, \\
L_{\epsilon}=\frac{-1}{8 \pi \kappa^{2}} \int \frac{1}{\epsilon^{2}} \epsilon,_{\mu},^{\mu} \sqrt{-g} d^{3} x .
\end{gathered}
$$

En un tratamiento cuántico donde se cuantiza al campo electromagnético la masa $m$ debe ser renormalizada tal que, $m=m_{0}+\delta m$, con $m_{0}$ independiente de $\epsilon$ (las expresiones $w_{0}$ representan a las magnitudes cuando $\epsilon=1$ ); y $\delta m$ puede ser calculada a través de la renormalización dada por la electrodimámica cuántica [113, 143],

$$
\delta m \approx \frac{3}{4 \pi} \epsilon^{2} \alpha_{0} m_{0} \log \left\{\frac{\Lambda^{2}}{m_{0}^{2}}\right\}
$$

Este término surge de las fluctuaciones cuánticas del campo electromagnético pero, como en nuestra aproximación los campos son clásicos, no estamos en condiciones de tratarlo (en la referencia [144] hay una buena descripción a partir de un método cuasi-clásico).

Sabiendo que $S_{i}=\int L_{i} d t$ encontramos las ecuaciones de Maxwell modificadas y de movimiento para el campo $\epsilon$ a partir del Principio de Mínima Acción, $\delta\left(S_{m}+S_{E M}\right) / \delta A_{\mu}=0$ y $\delta\left(S_{m}+S_{E M}+S_{\epsilon}\right) / \delta(\ln \epsilon)=0$ (la acción gravitatoria no depende de $A_{\mu}$ ni $\epsilon$ ) tal que,

$$
\begin{gathered}
\delta S_{E M}=\frac{-1}{8 \pi} \int F^{\mu \nu} \delta F_{\mu \nu} \sqrt{-g} d^{4} x \\
\delta F_{\mu \nu}=\epsilon^{-1}\left\{\epsilon_{, \mu} \delta A_{\nu}-\epsilon_{, \nu} \delta A_{\mu}+\epsilon\left[\left(\delta A_{\nu}\right)_{, \mu}-\left(\delta A_{\mu}\right)_{, \nu}\right]\right\} \\
\delta S_{E M}=\frac{-1}{4 \pi} \int F^{\mu \nu} \epsilon^{-1}\left(\epsilon A_{\nu}\right)_{, \mu} \sqrt{-g} d^{4} x
\end{gathered}
$$

$\mathrm{Al}$ integrar por partes y usando como hipótesis que $\delta A_{\nu}$ se anula en los extremos,

\footnotetext{
${ }^{1}\{-,+,+,+\}$ y $\kappa=\frac{\ell_{B}}{\sqrt{4 \pi \hbar c}}$. A $F^{\mu \nu}$ los reemplazamos por los campos electromagnéticos tal que, las componentes espacio temporales de $\epsilon F^{\mu \nu}$ representan al campo eléctrico $\mathbf{E}$, mientras que las componentes espacio-espacio se identifican con $\mathbf{B}$
} 
obtenemos:

$$
\delta S_{E M}=\frac{-1}{4 \pi} \int\left(F^{\mu \nu} \epsilon^{-1}\right)_{, \mu} \epsilon \delta A_{\nu} \sqrt{-g} d^{4} x .
$$

De igual manera calculamos la variación de la acción de la materia,

$$
\delta S_{m}=\int \epsilon j^{\mu} \delta A_{\mu} d^{4} x
$$

Luego, combinando las ecuaciones (3.26) y (3.27);

$$
\begin{gathered}
\frac{-1}{4 \pi} \int\left(F^{\mu \nu} \epsilon^{-1}\right)_{, \mu} \epsilon \delta A_{\nu} \sqrt{-g} d^{4} x+\int \epsilon j^{\mu} \delta A_{\mu} d^{4} x=0, \\
\left(F^{\mu \nu} \epsilon^{-1}\right)_{, \nu}=4 \pi j^{\mu},
\end{gathered}
$$

es fácil observar que cuando $\epsilon \rightarrow 1$ se recuperan las ecuaciones de Maxwell tradicionales.

Por otro lado para hallar la ecuación que describe al campo $\epsilon$ tenemos,

$$
\begin{gathered}
\delta S_{m}=\int-\frac{\partial}{\partial \epsilon}(\bar{\Psi} \delta m \Psi) \delta \epsilon d^{4} x+\int \frac{j^{\mu}}{\epsilon} A_{\mu} \delta \epsilon d^{4} x= \\
\int\left\{\epsilon j^{\mu} A_{\mu}-\epsilon \frac{\partial}{\partial \epsilon}(\bar{\Psi} \delta m \Psi)\right\} \delta \ln \epsilon d^{4} x, \\
\delta S_{E M}=\frac{-1}{8 \pi} \int F^{\mu \nu} \delta F_{\mu \nu} \sqrt{-g} d^{4} x \\
\delta F_{\mu \nu}=(\delta \ln \epsilon)_{, \mu} \delta A_{\nu}-(\delta \ln \epsilon)_{, \nu} \delta A_{\mu}, \\
\delta S_{E M}=\frac{1}{4 \pi} \int\left(A_{\nu} F^{\mu \nu}\right)_{, \mu} \delta \ln \epsilon \sqrt{-g} d^{4} x
\end{gathered}
$$

y por último,

$$
\begin{gathered}
\delta S_{\epsilon}=\frac{-1}{4 \pi \kappa^{2}} \int(\ln \epsilon)^{, \mu}(\delta \ln \epsilon)_{, \mu} \sqrt{-g} d^{4} x, \\
\delta S_{\epsilon}=\frac{1}{4 \pi} \int(\ln \epsilon)_{, \mu}^{, \mu} \delta \ln \epsilon \sqrt{-g} d^{4} x .
\end{gathered}
$$

Al sumar las expresiones (3.29), (3.30c) y (3.31b) podemos obtener el D'Alamber- 
tiano de $\ln \epsilon$,

$$
\square \ln \epsilon=4 \pi \kappa^{2}\left\{\epsilon \frac{\partial}{\partial \epsilon}(\bar{\Psi} \delta m \Psi)-\epsilon j^{\mu} A_{\mu}+\frac{1}{4 \pi}\left(A_{\mu} F^{\mu \nu}\right)_{, \nu}\right\} .
$$

Luego reemplazando $j^{\mu}$ por la expresión que hallamos en (3.28b), y desarrollando los términos de la última ecuación,

$$
\square \ln \epsilon=4 \pi \kappa^{2}\left\{\epsilon \frac{\partial}{\partial \epsilon}(\bar{\Psi} \delta m \Psi)-\frac{1}{8 \pi} F^{\mu \nu} F_{\mu \nu}\right\} .
$$

Entonces estudiando las variaciones espaciales del $\ln \epsilon$ para el caso de un sistema electrostático tenemos,

$$
\nabla^{2} \ln \epsilon=4 \pi \kappa^{2}\left\{\epsilon \frac{\partial}{\partial \epsilon}(\bar{\Psi} \delta m \Psi)+\frac{\epsilon^{-2} \mathbf{E}^{2}}{4 \pi}\right\} .
$$

Dado que, como ya explicamos anteriormente, nos encotramos limitados en el análisis del término $\epsilon \frac{\partial}{\partial \epsilon}(\bar{\Psi} \delta m \Psi)$, no estamos en condiciones de determinar si el desarrollo de Bekenstein para la contribución eléctrica (ver las subsecciones 1 y 2 de la sección 2.5) es válido o no, debido a que para ello, deberíamos cuantizar los campos actuantes en el modelo. Nosotros supondremos como correcta dicha afirmación y seguiremos analizando otros aspectos de la teoría.

\subsubsection{Comportamiento de la materia}

En esta parte de la tesis estudiaremos el tensor energía-impulso de la materia y sus "nuevos términos" debido a la presencia del campo escalar.

Trabajamos en un sistema de coordenadas localmente inercial ya que estamos considerando fenómenos locales. Llamaremos "tensor de energía-momento del campo", al tensor energía- momento del escalar más el tensor electromagnético $(\ln \epsilon=\psi$, y $\sigma=(\bar{\Psi} \delta m \Psi))$,

$$
\begin{gathered}
T_{\mathrm{f}}^{\mu \nu}=T_{\mathrm{em}}^{\mu \nu}+T_{\epsilon}^{\mu \nu} \\
T_{\mathrm{f}}^{\mu \nu}=\frac{1}{4 \pi}\left[F^{\mu \lambda} F_{\lambda}^{\nu}-\frac{1}{4} \eta^{\mu \nu} F_{\lambda \gamma} F^{\lambda \gamma}\right]+\frac{\hbar}{\ell_{B}^{2}}\left[\psi^{, \mu} \psi^{, \nu}-\frac{1}{2} \eta^{\mu \nu} \psi_{, \alpha} \psi^{, \alpha}\right] .
\end{gathered}
$$


Cuya divergencia está dada por,

$$
\begin{aligned}
T_{\mathrm{f}}^{\mu \nu}{ }_{, \nu}= & \frac{1}{4 \pi}\left[F_{, \nu}^{\mu \alpha} F_{\alpha}^{\nu}+F^{\mu \alpha} F^{\nu}{ }_{\alpha, \nu}-\frac{1}{2} \eta^{\mu \nu} F^{\alpha \beta} F_{\alpha \beta, \nu}\right] \\
& +\frac{\hbar}{\ell_{B}^{2}}\left[\psi^{, \mu}{ }_{, \nu} \psi^{, \nu}+\psi^{, \mu} \psi_{, \nu}{ }_{, \nu}-\eta^{\mu \nu} \psi_{, \alpha, \nu} \psi^{, \alpha}\right] .
\end{aligned}
$$

Introducimos las ecuaciones de movimiento que serán similares a las ecs. (2.10) y (2.11) en expresión anterior de manera de obtener,

$$
\begin{aligned}
T_{\mathrm{f}}^{\mu \nu}{ }_{, \nu}= & \frac{1}{4 \pi}\left[F_{, \nu}^{\mu \alpha} F_{\alpha}^{\nu}-F_{\alpha}^{\mu}\left(4 \pi e^{\psi} j^{\alpha}+\psi_{, \nu} F^{\alpha \nu}\right)\right. \\
& \left.-\frac{1}{2} \eta^{\mu \nu} F^{\alpha \beta} F_{\alpha \beta, \nu}\right] \\
& +\frac{\hbar}{\ell_{B}^{2}}\left[\psi_{, \nu}{ }_{, \nu} \psi^{, \nu}+\psi^{, \mu} \frac{\ell_{B}^{2}}{\hbar}\left(\frac{\partial \sigma}{\partial \psi}-\frac{F^{\mu \nu} F_{\mu \nu}}{8 \pi}\right)\right. \\
& \left.-\eta^{\mu \nu} \psi_{, \alpha, \nu} \psi^{, \alpha}\right] .
\end{aligned}
$$

La ecuación homogénea de Maxwell, $F_{\alpha \beta, \gamma}=-F_{\beta \gamma, \alpha}-F_{\gamma \alpha, \beta}$, nos ayudará a simplificar esta última ecuación cancelando el primer corchete. Además, se cancelan también el primer y el último término del segundo corchete pudiendo escribir la ec.(3.38) como,

$$
\begin{aligned}
T_{\mathrm{f}, \nu}^{\mu \nu}= & -e^{\psi} j^{\alpha} F_{\alpha}^{\mu} \\
& +\psi_{, \nu}\left(\eta^{\mu \nu} \frac{\partial \sigma}{\partial \psi}+T_{\mathrm{em}}^{\mu \nu}-\frac{1}{16 \pi} \eta^{\mu \nu} F_{\alpha \beta} F^{\alpha \beta}\right) .
\end{aligned}
$$

Ahora añadimos a ambos lados de la ecuación de la divergencia el tensor de energíamomento de la materia $T_{\mathrm{m}, \nu}^{\mu \nu}$ para poder encontrar la transferencia de energía (en concordancia con la hipótesis 8 del modelo de Bekenstein (Cap.2), suponemos que las ecuaciones de Einstein se mantienen sin ser modificadas para el campo gravitatorio; por lo tanto, el tensor energía-momento total se conserva)

$$
\begin{aligned}
& T_{\mathrm{f}, \nu}^{\mu \nu}+T_{\mathrm{m}, \nu}^{\mu \nu}=0 \\
& =T_{\mathrm{m}, \nu}^{\mu \nu}-e^{\psi} j^{\alpha} F_{\alpha}^{\mu} \\
& +\psi_{, \nu}\left(\eta^{\mu \nu} \frac{\partial \sigma}{\partial \psi}+T_{\mathrm{em}}^{\mu \nu}-\frac{1}{16 \pi} \eta^{\mu \nu} F_{\alpha \beta} F^{\alpha \beta}\right) .
\end{aligned}
$$


Como se puede observar, esta expresión describe explícitamente el intercambio de energía desde el campo $\psi$ a la materia,

$$
T_{\mathrm{m}, \nu}^{\mu \nu}=e^{\psi} j^{\alpha} F_{\alpha}^{\mu}-\psi_{, \nu}\left(\eta^{\mu \nu} \frac{\partial \sigma}{\partial \psi}+T_{\mathrm{em}}^{\mu \nu}-\frac{1}{16 \pi} \eta^{\mu \nu} F_{\alpha \beta} F^{\alpha \beta}\right),
$$

que es la fuente de cualquier efecto observable con la cual podemos verificar la validez del modelo.

A partir de la relación,

$$
\psi_{, \nu}=\frac{\epsilon_{, \nu}}{\epsilon}=\frac{1}{2} \frac{\alpha_{, \nu}}{\alpha},
$$

hallamos la contribución de energía anómala o "mágica" que se encuentra representada por,

$$
T_{\mathrm{m}, \nu}^{\mu \nu}{ }^{(\text {machian })}=\frac{1}{2} \frac{\alpha_{, \nu}}{\alpha}\left(\eta^{\mu \nu} \frac{\partial \sigma}{\partial \psi}+T_{\mathrm{em}}^{\mu \nu}-\frac{\eta^{\mu \nu}}{16 \pi} F_{\alpha \beta} F^{\alpha \beta}\right) .
$$

Si tomamos al sistema como un fluído, para analizar las fuerzas actuantes sobre el sistema utilizamos la ecuación de Euler que proyecta a la divergencia del tensor, $\nabla_{\beta} T_{T}^{\alpha \beta}$, en forma perperdicular a la tetravelocidad $u^{\alpha}$ tal que, para el límite no relativista $\partial_{0} T_{T}^{i 0}+\partial_{j} T_{T}^{i j}=0$. Nuevamente, los $F^{\mu \nu}$ son reemplazamos por los campos electromagnéticos utilizando la notación de Bekenstein; $v^{i}$ es la componente de la velocidad del fluído, $p$ la presión y $\rho_{m}$ densidad de masa. Por un lado hacemos,

$$
\begin{gathered}
\partial_{0} T_{T}^{i 0}+\partial_{j} T_{T}^{i j}=0 \\
\partial_{0} T_{m}^{i 0}+\partial_{j} T_{m}^{i j}=-\partial_{0} T_{\mathrm{g}}^{i 0}-\partial_{j} T_{\mathrm{g}}^{i j}, \\
-\partial_{0} T_{\mathrm{g}}^{i 0}-\partial_{j} T_{\mathrm{g}}^{i j}=-\partial_{0}\left(\rho_{m} v^{i}\right)-\partial_{j}\left(\rho_{m} v^{i} v^{j}+p \delta^{i j}\right), \\
\partial_{0} T_{m}^{i 0}=e^{\psi} j^{\alpha} F^{i}{ }_{\alpha}-\dot{\psi}\left(\eta^{i 0} \frac{\partial \sigma}{\partial \psi}+T_{e m}^{i 0}-\frac{\eta^{i 0} F_{\alpha \beta} F^{\alpha \beta}}{16 \pi}\right) \\
\partial_{0} T_{m}^{i 0}=e^{\psi} j^{\alpha} F^{i}{ }_{\alpha}-\dot{\psi} e^{-2 \psi} \frac{(\mathbf{E} \times \mathbf{B})_{i}}{4 \pi} .
\end{gathered}
$$

Mientras que por el otro,

$$
\partial_{j} T_{m}^{i j}=e^{\psi} j^{\alpha} F^{i}{ }_{\alpha}-\psi_{, j}\left\{\eta^{i j}\left[\frac{\partial \sigma}{\partial \psi}-\frac{1}{8 \pi} F_{\alpha \beta} F^{\alpha \beta}\right]+\frac{1}{4 \pi} F^{i \alpha} F^{j}{ }_{\alpha}\right\},
$$


Luego tenemos que,

$$
\begin{aligned}
\partial_{0} T_{m}^{i 0}+\partial_{j} T_{m}^{i j} & =2 e^{\psi} j^{\alpha} F^{i}{ }_{\alpha}-\dot{\psi} e^{-2 \psi} \frac{(\mathbf{E} \times \mathbf{B})_{i}}{4 \pi} \\
& -\psi_{, j}\left\{\eta^{i j}\left[\frac{\partial \sigma}{\partial \psi}-\frac{1}{8 \pi} F_{\alpha \beta} F^{\alpha \beta}\right]+\frac{1}{4 \pi} F^{i \alpha} F^{j}{ }_{\alpha}\right\} .
\end{aligned}
$$

En este momento, sólo nos interesa examinar las variaciones espaciales de $\psi$ y el caso de una distribución de carga arbitraria estática. Entonces, despreciaremos los términos que contengan $\dot{\psi}$ y supondremos que no hay campo magnético. Así, integrando en un volumen $\Omega$ la expersión (3.46),

$$
\begin{aligned}
\int_{\Omega} \partial_{0} T_{m}^{i 0} & +\partial_{j} T_{m}^{i j} d \Omega=\int_{\Omega} 2 e^{\psi} j^{\alpha} F_{\alpha}^{i} d \Omega \\
& \underbrace{-\int_{\Omega} \psi_{, j} \delta^{i j}\left[\frac{\partial \sigma}{\partial \psi}-\frac{1}{8 \pi} F_{\alpha \beta} F^{\alpha \beta}\right] d \Omega}_{=0^{(1)}} \\
& -\int_{\Omega} \psi_{, j} \frac{e^{-2 \psi}}{4 \pi} E_{i} E_{j} d \Omega .
\end{aligned}
$$

(1) suposición que surge de la subsección anterior). Utilizando las aproximaciones $e^{\psi} \approx e^{-2 \psi} \approx 1$ y $\nabla \psi$ pequeño, reescribimos la última ecuación,

$$
\int_{\Omega} \partial_{0} T_{m}^{i 0}+\partial_{j} T_{m}^{i j} d \Omega=2 \int_{\Omega} j^{\alpha} F^{i}{ }_{\alpha} d \Omega-\frac{1}{4 \pi} \int_{\Omega} \psi_{, j} E_{i} E_{j} d \Omega .
$$

La primer integral del lado derecho de la expresión es una fuerza tipo "Lorentz"; la segunda,

$$
-\frac{1}{4 \pi} \int_{\Omega} \psi_{, j} E_{i} E_{j} d \Omega=-\frac{1}{4 \pi} \int_{\Omega}(\vec{\gamma} \cdot \mathbf{E}) \cdot \mathbf{E} d \Omega
$$

siendo $\vec{\gamma}=\nabla \psi$. Trabajando en coordenadas esféricas,

$$
\begin{gathered}
\check{r}=\sin \theta \cos \phi \check{x}+\sin \theta \sin \phi \check{y}+\cos \theta \check{z}, \\
\check{\theta}=\cos \phi \cos \theta \check{x}+\cos \theta \sin \phi \check{y}-\sin \theta \check{z}, \\
\check{\phi}=-\sin \phi \check{x}+\cos \phi \check{y},
\end{gathered}
$$




$$
\begin{gathered}
\check{x}=\sin \theta \cos \phi \check{r}+\cos \phi \cos \theta \check{\theta}-\sin \phi \check{\phi}, \\
\check{y}=\sin \theta \sin \phi \check{r}+\cos \theta \sin \phi \check{\theta}+\cos \phi \check{\phi}, \\
\check{z}=\cos \theta \check{r}-\sin \theta \check{\theta},
\end{gathered}
$$

$$
\begin{gathered}
\check{\gamma}=\sin \theta_{\gamma} \cos \phi_{\gamma} \check{x}+\sin \theta_{\gamma} \sin \phi_{\gamma} \check{y}+\cos \theta_{\gamma} \check{z}, \\
\check{\theta_{\gamma}}=\cos \phi_{\gamma} \cos \theta_{\gamma} \check{x}+\cos \theta_{\gamma} \sin \phi_{\gamma} \check{y}-\sin \theta_{\gamma} \check{z}, \\
\check{\phi}_{\gamma}=-\sin \phi_{\gamma} \check{x}+\cos \phi_{\gamma} \check{y},
\end{gathered}
$$

tal que, $\vec{\gamma} \cdot \mathbf{E}=|\vec{\gamma}||\mathbf{E}| \cos \omega \operatorname{con}$

$$
\begin{aligned}
\cos \omega=\check{\gamma} \cdot \check{r} & =\sin \theta_{\gamma} \cos \phi_{\gamma} \sin \theta \cos \phi \\
& +\sin \theta_{\gamma} \sin \phi_{\gamma} \sin \theta \sin \phi+\cos \theta_{\gamma} \cos \theta
\end{aligned}
$$

Suponiendo simetría esférica, tenemos que analizar dos casos:

- Tomamos los campos generados por cuerpo en su exterior $\left(r>r_{0}\right.$ siendo $r_{0}$ el radio del cuerpo),

$$
\begin{aligned}
& -\frac{1}{4 \pi} \int_{\Omega}(\vec{\gamma} \cdot \mathbf{E}) \cdot \mathbf{E} d \Omega=-\frac{|\nabla \psi|}{4 \pi} \int_{r_{0}}^{\infty} \int_{0}^{2 \pi} \int_{0}^{\pi} \frac{Q_{T}^{2}}{r^{4}}\left(\sin \theta_{\gamma} \cos \phi_{\gamma} \sin \theta \cos \phi\right. \\
& \left.+\sin \theta_{\gamma} \sin \phi_{\gamma} \sin \theta \sin \phi+\cos \theta_{\gamma} \cos \theta\right) r^{2} \sin \theta d \theta d \phi d r= \\
& -\frac{1,5708|\nabla \psi|}{4 \pi} \int_{r_{0}}^{\infty} \int_{0}^{2 \pi} \frac{Q_{T}^{2}}{r^{2}}\left(\sin \theta_{\gamma} \cos \phi_{\gamma} \cos \phi+\sin \theta_{\gamma} \sin \phi_{\gamma} \sin \phi\right) d \phi d r=0
\end{aligned}
$$

- Consideramos los campos generados por el cuerpo dentro de él, el campo elétri- 
co es $\mathbf{E}=\rho_{C_{T}} r \check{r}$ siendo $\rho_{C_{T}}$ la densidad de carga total del cuerpo. Sea $r_{c}<r_{0}$

$$
\begin{aligned}
& -\frac{1}{4 \pi} \int_{\Omega}(\vec{\gamma} \cdot \mathbf{E}) \cdot \mathbf{E} d \Omega= \\
& -\frac{|\nabla \psi|}{4 \pi} \int_{r_{0}}^{\infty} \int_{0}^{2 \pi} \int_{0}^{\pi} \rho_{C_{T}}^{2} r^{2}\left(\sin \theta_{\gamma} \cos \phi_{\gamma} \sin \theta \cos \phi\right. \\
& \left.+\sin \theta_{\gamma} \sin \phi_{\gamma} \sin \theta \sin \phi+\cos \theta_{\gamma} \cos \theta\right) r^{2} \sin \theta d \theta d \phi d r= \\
& -\frac{\pi|\nabla \psi|}{8 \pi} \int_{r_{0}}^{\infty} \int_{0}^{2 \pi} \rho_{C_{T}}^{2} r^{4}\left(\sin \theta_{\gamma} \cos \phi_{\gamma} \cos \phi+\sin \theta_{\gamma} \sin \phi_{\gamma} \sin \phi\right) d \phi d r=0 .
\end{aligned}
$$

Por lo tanto probamos que,

$$
-\frac{1}{4 \pi} \int_{\Omega} \psi_{, j} E_{i} E_{j} d \Omega=0,
$$

esto vale tanto adentro como afuera, y la ecuación (3.48) queda bajo las aproximaciones usadas como,

$$
\int_{\Omega} \partial_{0} T_{m}^{i 0}+\partial_{j} T_{m}^{i j} d \Omega=2 \int_{\Omega} j^{\alpha} F^{i}{ }_{\alpha} d \Omega .
$$

No se observan fuerzas anómalas debido a la presencia del campo $\psi$.

\subsubsection{Contribución del campo magnético al campo escalar}

En un modelo cuántico que quiere describir a la materia, los campos magnéticos se generan no sólo a partir de las corrientes eléctricas estacionarias de partículas cargadas y sus momentos magnéticos estáticos, sino también de las fluctuaciones cuánticas de la "densidad número de partículas". La contribución principal a la energía magnética proviene de las oscilaciones nucleares dipolares con $T=1$. En éstas, protones y neutrones oscilan en antifase, con lo que se genera un momento dipolar variable, que origina una corriente variable. En lo que sigue, relacionaremos la corriente con los elementos de matriz de transición dipolares y con la regla de suma de Thomas-Reiche-Kuhn. Esta última está saturada (aproximadamente) por la resonancia dipolar gigante, bien medida sobre toda la tabla periódica. Las contribuciones 
a la energía magnética han sido calculadas por Haugan y Will [66, 145],

$$
E_{m}=\int d^{3} x \frac{B^{2}}{8 \pi} \simeq \frac{1}{2 c^{2}} \int d^{3} x d^{3} x^{\prime} \frac{\boldsymbol{j}(\boldsymbol{x}) \cdot \boldsymbol{j}\left(\boldsymbol{x}^{\prime}\right)}{\left|\boldsymbol{x}-\boldsymbol{x}^{\prime}\right|} \simeq \frac{3}{20 \pi} \frac{\hat{E}}{R(A) \hbar c} \int \sigma d E,
$$

donde $R(A), \hat{E}$ y $\int \sigma d E$ representan al radio nuclear, a la energía media absorbida en la resonancia gigante, y a la función de la fuerza integrada respectivamente. Estas cantidades las aproximamos por,

$$
R(A)=1,2 A^{\frac{1}{3}} \mathrm{fm}, \quad \hat{E} \sim 25 \mathrm{MeV}, \quad \int \sigma d E \simeq 1,6 A \mathrm{MeV} \mathrm{fm}^{2} .
$$

\section{Energía magnética nuclear}

Dado que las referencias anteriores no presentan una comprobación de la expresión para la energía magnética (3.58), nosotros la calculamos siguiendo los métodos utilizados por ellos. Empecemos por escribir la energía magnética total del núcleo,

$$
E_{m}=\frac{1}{2 c^{2}} \sum_{\alpha} \int d \boldsymbol{x} d \boldsymbol{x}^{\prime} \frac{\langle 0|\boldsymbol{j}(\boldsymbol{x})| \alpha\rangle \cdot\left\langle\alpha\left|\boldsymbol{j}\left(\boldsymbol{x}^{\prime}\right)\right| 0\right\rangle}{\left|\boldsymbol{x}-\boldsymbol{x}^{\prime}\right|}
$$

tal que $\alpha$ recorre un set completo de autoestados del Hamiltoniano nuclear $H$. Al operador de la corriente lo podemos definir como,

$$
\boldsymbol{j}(\boldsymbol{x})=\sum_{a} \delta\left(\boldsymbol{x}-\boldsymbol{x}_{a}\right) e_{a} \frac{\boldsymbol{p}_{a}}{m_{a}}
$$

donde la suma abarca a todas las partículas del sistema. Despreciamos la dependencia del momento del potencial nuclear para poder utilizar que,

$$
\frac{\boldsymbol{p}_{a}}{m_{a}}=\frac{i}{\hbar}\left[x_{a}, H\right]
$$

y hacer la sustitución en la ecuación anterior tal que,

$$
\begin{aligned}
\langle 0|\boldsymbol{j}(\boldsymbol{x})| \alpha\rangle & =\frac{i}{\hbar} \sum_{a} \delta\left(\boldsymbol{x}-\boldsymbol{x}_{a}\right)\left(E_{0}-E_{\alpha}\right)\left\langle 0\left|e_{a} \boldsymbol{x}_{a}\right| \alpha\right\rangle \\
& =\frac{i}{\hbar} \sum_{a} \delta\left(\boldsymbol{x}-\boldsymbol{x}_{a}\right)\left(E_{0}-E_{\alpha}\right) \boldsymbol{d}_{0 \alpha} .
\end{aligned}
$$


$\boldsymbol{d}(\boldsymbol{x})$ es el operador polarización (densidad dipolar) el cual, siempre y cuando se esté considerando una densidad nuclear constante, puede ser descripto como,

$$
\boldsymbol{d}_{0 \alpha}=\frac{d_{0 \alpha}}{V_{N}} \hat{\boldsymbol{x}}
$$

siendo $V_{N}=\frac{4 \pi}{3} R_{N}^{3}$ el volumen nuclear. Luego tenemos,

$$
\langle 0|\boldsymbol{j}(\boldsymbol{x})| \alpha\rangle \cdot\left\langle\alpha\left|\boldsymbol{j}\left(\boldsymbol{x}^{\prime}\right)\right| 0\right\rangle \simeq \frac{\left|d_{0 \alpha}\right|^{2}}{\hbar^{2}} \frac{E_{0 \alpha}^{2}}{V_{N}^{2}} \cos \theta,
$$

donde $\theta$ es el ángulo comprendido entre $\hat{\boldsymbol{x}}$ y $\hat{\boldsymbol{x}}^{\prime}$. De esta manera, encontramos una forma de expresar a la energía magnética,

$$
E_{m} \simeq \frac{\sum_{a} E_{0 \alpha}^{2}\left|d_{0 \alpha}\right|^{2}}{2 \hbar^{2} c^{2}} \frac{\int d \boldsymbol{x} d \boldsymbol{x}^{\prime} \frac{\cos \theta}{\left|\boldsymbol{x}-\boldsymbol{x}^{\prime}\right|}}{V_{N}^{2}}
$$

El último factor es igual a $\frac{3}{5 R_{N}}$; mientras que el primero se lo puede computar a partir de la conexión entre la función de fuerza y la sección eficaz de fotoabsorción,

$$
\sigma_{0 \alpha}=\frac{4 \pi}{\hbar c} E_{\alpha 0}\left|d_{\alpha 0}\right|^{2}
$$

Haciendo uso de lo anterior, llegamos a,

$$
\sum_{a} E_{\alpha 0}^{2}\left|d_{\alpha 0}\right|^{2}=\frac{\hbar c}{4 \pi} \frac{\int E \sigma(E) d E}{\int \sigma(E) d E} \cdot \int \sigma(E) d E=\bar{E} \int \sigma(E) d E
$$

$\bar{E} \sim 25 \mathrm{MeV}$ es la energía absorbida principal, que es independiente de $A$. Además, la sección eficaz satisface la regla de suma de Thomas-Reiche-Kuhn. Por lo tanto,

$$
\int \sigma(E) d E=(1+x) \frac{2 \pi^{2} e^{2} \hbar}{m c} \frac{N Z}{A} \simeq(1+x) 15 \mathrm{MeV} \operatorname{mbarn} A,
$$

donde $x \sim 0,2$ tiene en cuenta el intercambio y la velocidad de dependencia de las interacciones nucleares. Combinamos las ecuaciones (3.66), (3.68) y (3.69) de manera tal de obtener las expresiones (3.58) y (3.59) que son las que nos interesan. 


\section{Energía magnética de la materia}

Debido a que la densidad de energía magnética está concentrada cerca del núcleo atómico, puede ser representada por,

$$
e_{m}(\boldsymbol{x})=\sum_{a} E_{m}^{a} \delta\left(\boldsymbol{x}-\boldsymbol{x}_{a}\right) \simeq \sum_{b} E_{m}^{b} n_{b}(\boldsymbol{x})
$$

donde $b$ tiene en cuenta las diferentes especies nucleares y definimos a,

$$
\zeta_{m}^{b}=\frac{E_{m}^{b}}{M_{b} c^{2}}
$$

como la contribución fraccional de la energía magnética a la masa en reposo. De esta manera,

$$
e_{m}(\boldsymbol{x})=\bar{\zeta}_{m}(\boldsymbol{x}) \rho(\boldsymbol{x}) c^{2}
$$

tal que $\rho(\boldsymbol{x})$ es la densidad de masa local y

$$
\bar{\zeta}_{m}(\boldsymbol{x})=\frac{\sum_{b} \zeta_{b} \rho_{b}(\boldsymbol{x})}{\rho(\boldsymbol{x})}
$$

es el promedio pesado de $\zeta_{m}$.

Empleando la expresión (3.72) podemos escribir una ecuación semejante a la presentada anteriormente (2.43) de la siguiente forma,

$$
\nabla^{2} \psi=-8 \pi \kappa^{2} c^{2} e^{-2 \psi} \bar{\zeta}_{m} \rho
$$

En la aproximación de $\psi$ pequeño nos es posible hallar una solución para una distribución arbitraria de fuentes,

$$
\psi=8 \pi \kappa^{2} c^{2} \frac{1}{r} \int_{0}^{r} x^{2} \bar{\zeta}_{m}(x) \rho(x) d x
$$

cuyo comportamiento asintótico lo describimos en términos del potencial gravitatorio newtoniano

$$
\psi \asymp \frac{8 \pi \kappa^{2}}{G M} \phi_{N}(r) \int_{0}^{\infty} x^{2} \bar{\zeta}_{m}(x) \rho(x) d x=2\left(\frac{\ell_{B}}{\ell_{P}}\right)^{2} \tilde{\zeta}_{m} \frac{\phi_{N}(r)}{c^{2}}
$$


siendo $\tilde{\zeta}_{m}$ el valor de la masa promedio de $\zeta_{m}$.

\subsection{Resultados}

Anteriormente, hemos mostrado que en un modelo semiclásico con una densidad de carga arbitraria, acotada, cuántica y estática, podemos considerar como "válida" (en realidad no podemos determinar si es correcta o no) la propuesta de Bekenstein y por lo tanto, tanto el campo eléctrico como la masa no contribuyen al campo escalar $\psi$. Esta "cancelación" del campo eléctrico no ocurre para el campo magnético, tal que puede observarse un término anómalo en la aceleración producto de la contribución magnética generada por las fluctuaciones cuánticas que sufren los nucleones.

De la expresión (3.76) obtenemos que para la diferencia de aceleraciones entre dos cuerpos distintos $A y$ s se cumple,

$$
\eta(A, B)=\frac{a_{A}-a_{B}}{g}=4\left(\frac{\ell_{B}}{\ell_{P}}\right)^{2} \zeta_{S}\left(\zeta_{A}-\zeta_{B}\right)=C_{f}\left(\frac{\ell_{B}}{\ell_{P}}\right)^{2},
$$

en este caso, $\zeta_{S}, \zeta_{A}, \zeta_{B}$ son las fracciones de energía magnética de la fuente y de los cuerpos $A$ y $B$ respectivamente.

$$
\zeta_{i}=\frac{E_{m i}}{M_{i} c^{2}}
$$

Utilizamos los resultados obtenidos para las versiones más precisas de los experimentos tipo Eötvös ${ }^{2}$ que se muestran en la tabla 3.1 para realizar un ajuste estadístico con el modelo $y=C_{f} x^{2}$ tal que,

$$
\left(\frac{\ell_{B}}{\ell_{P}}\right)^{2}=0,0003 \pm 0,0006
$$

de donde a " $3 \sigma$ " se alcanza el límite superior

$$
\left(\frac{\ell_{B}}{\ell_{P}}\right)^{2}<0,002 \quad \frac{\ell_{B}}{\ell_{P}}<0,05 .
$$

A partir de estos resultados, podemos concluir que aún cuando suponemos que el

\footnotetext{
${ }^{2}$ más adelante explicaremos con más detalle la UFF y los experimentos de Eötت̈os
} 
campo electrostático no contribuye al campo escalar $\psi$, las contribuciones del campo magnético producen violaciones al Principio de Equivalencia, esto se evidencia gracias a las cotas que imponen los experimentos de Eötvös al párametro propuesto por Bekenstein $\ell_{B} / \ell_{P}$. Estas cotas encontradas son mayores a las que se pudieran hallar si el densidad de energía electrostática pudiese generar la intensidad del campo $\psi$; este cálculo fue llevado a cabo por el mismo Bekenstein [15] y repetido varias veces $[47,96]$, obteniendo como resultado,

$$
\left(\frac{\ell_{B}}{\ell_{P}}\right)_{\mathrm{el}}<8,7 \times 10^{-3},
$$

un orden de magnitud más pequeño que (3.80).

Es muy interesante comparar el resultado de la ecuación (3.80) con los resultados que se obtuvieron gracias al ánalisis de todas las evidencias de la variación temporal de la constante de estructura fina $\alpha$ detallado en la referencia [96]. En este artículo, se utilizó un valor efectivo de $\zeta=10^{-4}$, siguiendo como sugerencia al artículo [67]; tal que $1 \sigma$, el límite es de $\left(\ell_{B} / \ell_{P}\right)^{2}<0,003$. Utilizando el mismo $\zeta_{\mathrm{H}}$ que en el modelo propuesto por Bekenstein en 2002 [16] nosotros llegamos a un valor de $\zeta_{U}$ efectivo de $2,7 \times 10^{-5} \Omega_{B} \simeq 1,4 \times 10^{-6}$, y luego hallamos a $3 \sigma$ una cota superior de,

$$
\frac{\ell_{B}}{\ell_{P}}<0,8
$$

un orden de magnitud más grande que el resultado (3.80). 


\begin{tabular}{cccccr}
\hline$A$ & $B$ & Fuente & $10^{11} C_{f}$ & $10^{11} \eta(A, B)$ & Ref. \\
\hline $\mathrm{Al}$ & $\mathrm{Au}$ & Sol & 17.5 & $1.0 \pm 1.5$ & {$[119]$} \\
$\mathrm{Al}$ & $\mathrm{Pt}$ & Sol & 17.5 & $0.03 \pm 0.045$ & {$[19]$} \\
$\mathrm{Cu}$ & $\mathrm{W}$ & Sol & 8.8 & $0.0 \pm 2.0$ & {$[75]$} \\
$\mathrm{Be}$ & $\mathrm{Al}$ & Tierra & 6.8 & $-0.02 \pm 0.23$ & {$[130]$} \\
$\mathrm{Be}$ & $\mathrm{Cu}$ & Tierra & 10.4 & $-0.19 \pm 0.25$ & {$[130]$} \\
$\mathrm{Be}$ & $\mathrm{Al}$ & Sol & 16.1 & $0.40 \pm 0.98$ & {$[130]$} \\
$\mathrm{Be}$ & $\mathrm{Cu}$ & Sol & 24.6 & $-0.51 \pm 0.61$ & {$[130]$} \\
$\mathrm{Si} / \mathrm{Al}$ & $\mathrm{Cu}$ & Sol & 8.8 & $0.51 \pm 0.67$ & {$[130]$} \\
$\mathrm{EC}$ & $\mathrm{MM}$ & Sol & -7.6 & $0.001 \pm 0.032$ & {$[51]$} \\
$\mathrm{Be}$ & $\mathrm{Ti}$ & Tierra & 6.9 & $0.004 \pm 0.018$ & {$[122]$} \\
\hline
\end{tabular}

Cuadro 3.1: Resultados de experimentos tipo Eötvös. Las columnas muestran la composición de los cuerpos, la fuente del campo gravitatorio, el coeficiente $\left(\ell_{B} / \ell_{P}\right)^{2}$ de la ec. (3.77), el valor medido de $\eta$ y su error a un $1 \sigma$, y las referencias. 


\section{Capítulo 4}

\section{Intercambio de energía en la teoría de Bekenstein}

La variación temporal de la constante de estructura fina $\alpha$ ha sido estudiada como ya mecionamos en la introducción, en varias ocasiones desde la teoría propuesta por Gamow [61]. Tal es así, que se pueden encontrar numerosos artículos en los cuales se publican cotas superiores estimadas a partir de datos observacionales, así como también modelos teóricos que consideran a $\alpha$ como un campo dinámico (se puede encontrar una lista completa en [86, 134] y las referencias allí), y la afirmación de Webb y sus colaboradores [105], quienes declaran que $\alpha$ fue menor.

El modelo de Bekenstein descripto anteriormente en el capítulo 2, considera ambos tipos de variación: la temporal de $\alpha$ (sección 2.5), y la espacial (secciones 2.1, $2.2,2.3,2.4)$. Nosotros en este capítulo analizaremos las ecuaciones que describen el intercambio de energía entre la materia y los campos electromagnético y escalar $\psi$. Si bien no prestaremos demasiada atención al mecanismo preciso de la liberación de energía, supondremos que el trabajo realizado por el campo escalar se irradia de una manera eficiente, como el calor por efectos rotoquímicos en las estrellas de neutrones debido al "spin down" de la estrella [53, 118].

En el artículo publicado por Bekenstein [15] (releer la subsección 2.4 del capítulo 2 para un mayor detalle, aquí sólo se refrescarán algunas ecuaciones y nociones 
básicas), se muestra que la ecuación de movimiento cosmológica para $\epsilon$ es,

$$
\frac{d}{d t}\left(a^{3} \frac{\dot{\epsilon}}{\epsilon}\right)=-a^{3} \frac{\ell_{B}^{2}}{\hbar c}\left[\epsilon \frac{\partial \sigma}{\partial \epsilon}-\frac{1}{4 \pi}\left(\mathbf{E}^{2}-\mathbf{B}^{2}\right)\right] .
$$

En un régimen no relativista se cumple, $\mathbf{E}^{2} \gg \mathbf{B}^{2}$ y $\sigma \propto \epsilon^{2}$, entonces

$$
\frac{d}{d t}\left(a^{3} \frac{\dot{\epsilon}}{\epsilon}\right)=-a^{3} \zeta_{c} \frac{\ell_{B}^{2}}{\hbar c} \rho_{m} c^{2},
$$

tal que $\rho_{m}$ es la densidad total de masa en reposo de materia que interactúa electromagnéticamente, y $\zeta_{c}$ es el parámetro que describe su "contenido eletromagnético" ya que es la contribución fraccionaria de la energía eletromagnética a la energía de la masa en reposo. Una primera estimación de acuerdo con [16] es

$$
\zeta_{c} \sim 1,2 \times 10^{-3} .
$$

Siguiendo al modelo standard cosmológico, se supone que la materia oscura es electromagnéticamente neutra. Luego, tomando $\rho_{m} \propto a^{-3}$, se puede integrar la expresión (4.2) tal que, reescribiéndola en notación cosmológica,

$$
\frac{\dot{\epsilon}}{\epsilon}=-\frac{3 \zeta_{c}}{8 \pi}\left(\frac{\ell_{B}}{\ell_{P}}\right)^{2} H_{0}^{2} \Omega_{B}\left[\frac{a_{0}}{a(t)}\right]^{3}\left(t-t_{c}\right) .
$$

La constante de integración $t_{c}$ debe ser muy pequeña de manera tal de no entorpecer el acuerdo entre la teoría y las observaciones. Utilizando los valores WMAP, se llegan a estimaciones para $(\dot{\alpha} / \alpha)_{0}$ del orden de

$$
\left(\frac{\dot{\alpha}}{H_{0} \alpha}\right)_{0}=1,3 \times 10^{-5}\left(\frac{\ell_{B}}{\ell_{P}}\right)^{2},
$$

siendo muy difícil de lograr una comparación entre la teoría y los datos observacionales como se menciona en el capítulo 2.

Los mismos argumentos se pueden aplicar a otras teorías para la variación de $\alpha$, como las del tipo Kaluza-Klein [86] o las de Cuerdas inspiradas en modelos como los de Damour y Polyakov [37, 38]. 


\subsection{Energía transferida}

Estudiaremos cómo la energía es inyectada y luego liberada en la teoría de $\alpha$ variable en el tiempo (sección 2.4), con el fin de poder hallar consecuencias observables en la emisión de los sistemas astrofísicos, así como geofísicos. Para ello, debemos retomar el análisis del tensor energía-impulso propuesto en el capítulo anterior (subsección 3.2.1).

La contribución dada por la ec.(3.43) puede ser escrita en función de los campos electromagnéticos;

$$
\begin{aligned}
& T_{\mathrm{m}, \nu}^{0 \nu}{ }^{(\text {machian })}=-\dot{\psi} \frac{\partial \sigma}{\partial \psi}+e^{-2 \psi} \nabla \psi \cdot \mathbf{S}+\dot{\psi} e^{-2 \psi} \frac{\left(\mathbf{B}^{2}+\mathbf{E}^{2}\right)}{8 \pi} \\
& +\frac{e^{-2 \psi} \dot{\psi}}{8 \pi}\left(\mathbf{B}^{2}-\mathbf{E}^{2}\right) \\
& =-\dot{\psi} \frac{\partial \sigma}{\partial \psi}+e^{-2 \psi} \nabla \psi \cdot \mathbf{S}+\dot{\psi} e^{-2 \psi} \frac{\mathbf{B}^{2}}{4 \pi},
\end{aligned}
$$

donde $\mathbf{S}=\frac{\mathbf{E} \times \mathbf{B}}{4 \pi}$. Luego, la componente 0 de la ec.(3.41) queda,

$$
T_{\mathrm{m}, \nu}^{0 \nu}=\mathbf{j} . \mathbf{E}-e^{-2 \psi} \frac{\mathbf{B}^{2} \dot{\psi}}{4 \pi}-e^{-2 \psi} \nabla \psi \cdot \mathbf{S}+\dot{\psi} \frac{\partial \sigma}{\partial \psi}
$$

Utilizando nuestro análisis anterior podemos desarrollar una versión "generalizada" del teorema de Poynting. En su versión standard, este teorema sólo tiene en cuenta términos provenientes del electromagnetismo, pero en este caso hay que sumarle la interacción entre los campos escalar y electromagnético tal que,

$$
\begin{aligned}
T_{\mathrm{em}, \nu}^{\mu \nu}= & \frac{1}{4 \pi}\left[F_{, \nu}^{\mu \alpha} F_{\alpha}^{\nu}-F_{\alpha}^{\mu}\left(4 \pi e^{\psi} j^{\alpha}+\psi_{, \nu} F^{\alpha \nu}\right)\right. \\
& \left.-\frac{1}{2} \eta^{\mu \nu} F^{\alpha \beta} F_{\alpha \beta, \nu}\right] .
\end{aligned}
$$

Haciendo uso de la ecuación homogénea de Maxwell,

$$
\begin{aligned}
T_{e m, \nu}^{\mu \nu}= & -e^{\psi} j^{\alpha} F_{\alpha}^{\mu} \\
& +\psi_{, \nu}\left[\frac{F^{\mu}{ }_{\alpha} F^{\nu \alpha}}{4 \pi}-\eta^{\mu \nu} \frac{F_{\alpha \beta} F^{\alpha \beta}}{16 \pi}+\eta^{\mu \nu} \frac{F_{\alpha \beta} F^{\alpha \beta}}{16 \pi}\right],
\end{aligned}
$$




$$
T_{e m, \nu}^{\mu \nu}=-e^{\psi} j^{\alpha} F_{\alpha}^{\mu}+\psi_{, \nu}\left(T_{e m}^{\mu \nu}+\eta^{\mu \nu} \frac{F_{\alpha \beta} F^{\alpha \beta}}{16 \pi}\right) .
$$

De esta forma se llega a,

$$
\begin{aligned}
T_{e m, \rho}^{0 \rho}= & -\mathbf{E} \cdot \mathbf{j}+e^{-2 \psi} \frac{\left(\mathbf{E}^{2}+\mathbf{B}^{2}\right)}{8 \pi} \dot{\psi} \\
& +e^{-2 \psi} \mathbf{S} . \nabla \psi-\frac{e^{-2 \psi} \dot{\psi}}{8 \pi}\left(\mathbf{B}^{2}-\mathbf{E}^{2}\right), \\
T_{e m}^{0 \rho}{ }_{, \rho}= & \frac{\partial u_{e m}}{\partial t}+\nabla \cdot e^{-2 \psi}\left(\frac{\mathbf{E} \times \mathbf{B}}{4 \pi}\right) \\
& =-\mathbf{E} \cdot \mathbf{j}+\frac{e^{-2 \psi} \mathbf{E}^{2}}{4 \pi} \dot{\psi}+e^{-2 \psi} \mathbf{S} . \nabla \psi,
\end{aligned}
$$

donde $T_{e m}{ }^{00}{ }_{0}=\left(\partial u_{e m}\right) / \partial t$. La energía electromagnética es $u_{e m}=e^{-2 \psi}\left(\mathbf{E}^{2}+\mathbf{B}^{2}\right) /(8 \pi)$, $T_{e m}{ }^{0 i}{ }_{i}=\nabla \cdot e^{-2 \psi}\left(\frac{\mathbf{E} \times \mathbf{B}}{4 \pi}\right)=\nabla \cdot e^{-2 \psi} \mathbf{S}$, y $\mathbf{S}$ representa al vector de Poynting. Este resultado que se obtiene es independiente de los Lagrangianos de materia y de campo gravitatorio. En particular, se mantiene a parte de la interacción entre la materia y el campo escalar acoplado. Recordemos que la interpretación usual del primer término el lado derecho de la ecuación (4.12) es el trabajo realizado por el campo electromagnético sobre la materia. De la misma manera, podemos interpretar al segundo y al último término como el trabajo realizado por el campo electromagnético sobre el campo escalar. Un fenómeno análogo sería el dado por el trabajo realizado por el incremento de la constante de Newton $G$ en un planeta que aumenta su presión y por ende se comprime [72].

Para estimar la contribución electrostática a la energía de materia, consideramos un sistema no relativista como un átomo liviano o un núcleo, tal que la energía electromagnética dada por el campo eléctrico cumple con la condición,

$$
\boldsymbol{\nabla} \cdot \boldsymbol{E} e^{-2 \psi}=4 \pi \rho_{\mathrm{em}}^{0}
$$

donde $\rho_{\mathrm{em}}^{0}$ es la densidad de carga de referencia. En el límite donde $\alpha$ solo varía cosmológicamente se tiene,

$$
\begin{gathered}
\boldsymbol{\nabla} \cdot \boldsymbol{E}=4 \pi e^{2 \psi} \rho_{\mathrm{em}}^{0} \cdot \\
\boldsymbol{E}=e^{2 \psi} \boldsymbol{E}_{0}
\end{gathered}
$$


Se puede observar que la solución que encontramos está dada en términos de $\boldsymbol{E}_{0}$ que es el campo electrostático de referencia definido para $e^{\psi}=1$. Luego, estamos en condiciones de describir a la energía electromagnética como,

$$
u_{e m}=e^{-2 \psi} \frac{\left(\mathbf{B}^{2}+\mathbf{E}^{2}\right)}{8 \pi}=e^{2 \psi} u_{e m}^{0}
$$

y así su variación temporal,

$$
\dot{u}_{e m}=2 \dot{\psi} u_{e m}+e^{2 \psi} \dot{u}_{e m}^{0}=\frac{\dot{\alpha}}{\alpha} u_{e m}+e^{2 \psi} \dot{u}_{e m}^{0} .
$$

Si no llegase a existir una inyección escalar de energía y $\dot{u}_{e m}^{0} \approx 0$; el teorema de Poynting y la variación de energía, mostrados en las ecuaciones (4.12) y (4.17) respectivamente, darían lugar a,

$$
2 \dot{\psi} u_{e m}=2 \dot{\psi} e^{-2 \psi} \frac{\left(\mathbf{B}^{2}+\mathbf{E}^{2}\right)}{8 \pi}=-\boldsymbol{j} \cdot \boldsymbol{E}+\dot{\psi} e^{-2 \psi} \frac{\mathbf{E}^{2}}{4 \pi} .
$$

Simplificando,

$$
\boldsymbol{j} \cdot \boldsymbol{E}=-\frac{\mathbf{B}^{2}}{4 \pi} \dot{\psi} e^{-2 \psi} .
$$

Estamos considerando un sistema en donde se desprecia al movimiento de la materia. Consecuentemente, es equivalente tomar como 0 al primer índice, que proyectar a lo largo de la cuadru-velocidad del fluído. Además, la derivada total en el tiempo $d / d t=\partial / \partial t+\mathbf{v} . \nabla$ es igual a la derivada parcial respecto al tiempo $\partial / \partial t$. En el caso general, cuando existen los fenómenos de viscosidad y transferencia de calor, el lado derecho de la ecuación puede expresarse en el límite no relativista como,

$$
T_{\mathrm{m}, \nu}^{0 \nu}=\frac{\partial}{\partial t}\left(\frac{1}{2} \rho v^{2}+u\right)+\nabla \cdot\left[\rho \mathbf{v}\left(\frac{1}{2} v^{2}+w\right)-\mathbf{v} \cdot \sigma^{\prime}+\mathbf{J}\right] .
$$

La entalpía específica y la densidad de energía interna están dadas por $w$ y $u$. J es el flujo de calor, que generalmente puede escribirse como $-\kappa \nabla T$, siendo $T$ la temperatura y $\kappa$ la conductividad termal. Por último, $\left(\mathbf{v} \cdot \sigma^{\prime}\right)_{k}$ lo podemos representar mediante $v_{i} \sigma_{i k}^{\prime}$, donde $\sigma^{\prime}$ es el tensor de esfuerzo viscoso, [83]. Luego obtenemos,

$$
T_{\mathrm{m}, \nu}^{0 \nu}=\frac{\partial u}{\partial t}+\nabla \cdot \mathbf{J}
$$


Nosotros entendemos por "energía interna" a aquella energía que puede ser intercambiada por el sistema en los procesos como por ejemplo, intercambio de calor, transferencia de radiación, etc. Ésta será diferente de la que entendemos por " masa en reposo", que es la "energía no convertible". Si el campo escalar puede cambiar la carga eléctrica efectiva, entonces se puede alterar la contribución electromagnética a la masa en reposo, y por lo tanto, esta contribución no será más "masa en reposo" sino "energía interna".

El cambio temporal de la energía interna $u$ tendrá dos contribuciones: la primera corresponde al aporte del proceso de enfriamiento $\left.\frac{\partial u}{\partial t}\right|_{\text {cooling, y la otra, está rela- }}$ cionada con la interacción con el campo escalar, $\frac{\partial \sigma_{\mu}}{\partial t}$. Este último término explica la dependencia de la mayor parte de la materia con el campo $\psi$, que principalmente está constituida por la contribución electromagnética a la masa nuclear. Finalmente, la ecuación (4.7) queda

$$
\begin{aligned}
& \left.\frac{\partial u}{\partial t}\right|_{\text {cooling }}+\frac{\partial \sigma_{\mu}}{\partial t}+\nabla \cdot \mathbf{J}= \\
& -\frac{\boldsymbol{B}^{2}}{4 \pi} \dot{\psi} e^{-2 \psi}-\frac{e^{-2 \psi} \mathbf{B}^{2} \dot{\psi}}{4 \pi}-e^{-2 \psi} \nabla \psi \cdot \mathbf{S}-\dot{\psi} \frac{\partial \sigma}{\partial \psi} .
\end{aligned}
$$

Dado que para este caso consideramos al campo escalar independiente del espacio, y por ende, la energía electromagnéticade la materia se halla principalmente en el contenido nuclear, estamos en condiciones de suponer que se cumple la condición $\frac{\partial \sigma}{\partial \psi}-\frac{\partial \sigma_{\mu}}{\partial \psi} \approx 0$, arrivando a

$$
\left.\frac{\partial u}{\partial t}\right|_{\text {cooling }}+\nabla \cdot \mathbf{J} \approx-\frac{e^{-2 \psi} \mathbf{B}^{2} \dot{\psi}}{2 \pi} .
$$

Esta ecuación se simplifica si se hace un cambio trivial para obtener,

$$
\nabla \cdot \mathbf{J}=-\frac{e^{-2 \psi} \mathbf{B}^{2} \dot{\psi}}{2 \pi}-\left.\frac{\partial u}{\partial t}\right|_{\text {cooling }}
$$

de la cual es posible observar claramente que aparte del mecanismo de enfriamiento standard del cuerpo, aparece una contribución de energía magnética liberada debido 
a la interacción con el campo $\psi$. Definimos,

$$
\varepsilon_{a}=2 \frac{e^{-2 \psi} \mathbf{B}^{2} \dot{\psi}}{M_{a} 4 \pi} \approx 2 \frac{\dot{\alpha}}{\alpha} \frac{\mathbf{B}^{2}}{8 \pi M_{a}}
$$

que es igual a dos veces la producción de energía por unidad de masa de cualquier substancia a (bajo la aproximación de $e^{-2 \psi} \rightarrow 1$ cuando $\psi<<1$ ).

Nuestra principal hipótesis es que "el término de enfriamiento no se ve modificado por el campo escalar". Las razones que tenemos para hacer semejante suposición son: 1) como ya hemos mostrado antes, la energía electrostática inyectada por el campo escalar permanece en el "grueso" de la materia (la cancelación de los términos se pueden observar en la ec.(4.23)); 2) la evolución termal no debe cambiar dada la alta conductividad térmica de la Tierra y de otros planetas que serán considerados en este trabajo más adelante. De esta forma, esperamos que el exceso de energía magnética irradiada aumente el flujo de calor J como se observa en la expresión (4.24).

\subsubsection{La energía electromagnética de la materia}

Como ya mencionamos anteriormente, la única contribución anómala se obtiene a partir del campo magnético. En la sección 3.2.2, vimos que las corrientes eléctricas estacionarias generadas por las partículas cargadas y sus momentos magnéticos estáticos, y las fluctuaciones cuánticas de la densidad del número son los responsables de la generación de campos magnéticos en la mecánica cuántica. Estas contribuciones han sido estudiadas y calculadas por $[66,145]$ para un modelo de capas nucleares mínimas, y desarrolladas ampliamente en el capítulo 3, sección 3.2.2.

Luego, la contribución fraccionaria de la energía magnética a la energía de masa en reposo para una substancia de número másico $A$ es,

$$
\zeta(A) \approx 8,60465 \times 10^{-6} A^{-1 / 3}
$$

La tabla 4.1 muestra valores típicos de $\zeta(A)$ que se obtuvieron gracias a la fórmula semi-empírica de masas y las contribuciones de neutrones y protones. 


\begin{tabular}{|c|c|}
\hline Núcleo & $10^{6} \zeta$ \\
\hline${ }_{2}^{4} \mathrm{He}$ & 5,42 \\
${ }_{6}^{12} \mathrm{C}$ & 3,76 \\
${ }_{8}^{16} \mathrm{O}$ & 3,41 \\
${ }_{14}^{28} \mathrm{Si}$ & 2,83 \\
${ }_{26}^{56} \mathrm{Fe}$ & 2,25 \\
\hline
\end{tabular}

Cuadro 4.1: Valores de $\zeta$ para elementos típicos de interiores de estrellas y planetas

\subsection{Flujos de calor planetarios}

En esta sección calcularemos el flujo de calor de distintos planetas, para obtener cotas superiores a las variaciones temporales de $\alpha$, y verificar así la veracidad del modelo de Bekenstein.

\subsubsection{Flujo de calor terrestre}

Existen varios modelos que intentan explicar la tasa media de enfriamiento secular de la Tierra en términos de variaciones en la composición del manto se funde en el tiempo [82]. Las limitaciones de estas teorías están establecidas por las mediciones terrestres de flujo de calor en la superficie. La contribución de $\dot{\alpha} / \alpha$ al flujo de calor puede ser calculado haciendo uso del balance de calor global de la Tierra, suponiendo que la contribución "mágica", $H_{C}$ es la única producción de energía extra, tenemos,

$$
M_{E} C_{p} \frac{d T_{m}}{d t}=-Q_{t o t}+H_{C}+H_{G},
$$

donde $M_{E}$ y $C_{p}$ representan la masa de la Tierra y la capacidad calorífica promedio del planeta. Además, $T_{m}$ es la temperatura potencial del manto, y $H_{G}$ encarna al calor generado por isótopos radioactivos. La pérdida total de calor $Q_{\text {tot }}$ la podemos escribir como la suma de un término que está relacionado con la pérdida de calor por los océanos $Q_{o c}$, y otro que tiene que ver con la pérdida del calor continental $Q_{\text {cont }}$. Utilizando los resultados de la referencia Labrosse y Jaupart [82], reescribimos la pérdida total de calor como $Q_{t o t} \approx M C_{p} \lambda_{G} T_{m}$, tal que $\lambda_{G}$ es el tiempo de escala 
constante para el enfriamiento secular de la Tierra. Suponemos que los elementos más abundantes en la costitución de nuestro planeta son el Oxígeno, el Silicio y el Hierro, consecuentemente, llegamos a la siguiente expresión para la contribución "extra" de energía,

$$
H_{C}=\bar{\zeta} c^{2} H_{0} \frac{\dot{\alpha}}{\alpha H_{0}},
$$

$\bar{\zeta}$ es el promedio pesado de la masa del parámetro $\zeta(A)$.

\begin{tabular}{|c|c|}
\hline Parámetro & Valor \\
\hline $\mathrm{H}_{0}$ & $2,5 \times 10^{-18} \mathrm{~s}^{-1}$ \\
$\mathrm{M}_{\mathrm{E}}$ & $5,94 \times 10^{24} \mathrm{Kg}$ \\
$\mathrm{C}_{\mathrm{P}}$ & $1200 \mathrm{~J} / \mathrm{Kg}-\mathrm{K}$ \\
$\bar{\zeta}$ & $2,75 \times 10^{-6}$ \\
$\lambda_{\mathrm{G}}$ & $0,1 \mathrm{Gyr}^{-1}$ \\
\hline
\end{tabular}

Cuadro 4.2: Valores de los parámetros

Gracias a la ecuación (2.21), podemos describir a la contribución extra como función del tiempo, desarrollando $\frac{a(t)}{a_{0}}$ en serie de potencias, [141]

$$
\frac{a(t)}{a_{0}} \approx 1+H_{0} d t-\frac{q_{0}}{2}\left(H_{0} d t\right)^{2}+\frac{j_{0}}{6}\left(H_{0} d t\right)^{3}+\cdots
$$

y tomando hasta el tercer orden de $H_{C}$ en la expansión de Taylor. Reemplazamos la contribución "mágica" en la expresión (4.27), y resolvemos usando los valores de los parámetros de la Tabla 4.2, tal que encontramos la perturbación cosmológica de la temperatura del manto $\Delta T_{m}$ en términos del intervalo de tiempo $\Delta t$ y $\frac{\dot{\alpha}}{\alpha H_{0}}$,

$$
\begin{aligned}
& \Delta T_{m}(t)=2,43 \times 10^{5} \mathrm{~K} / \mathrm{Gyr} \frac{\dot{\alpha}}{H_{0} \alpha}(\Delta t)^{3} \\
& -3,78 \times 10^{6} \mathrm{~K} / \mathrm{Gyr} \frac{\dot{\alpha}}{H_{0} \alpha}(\Delta t)^{2} \\
& +3,05 \times 10^{7} \mathrm{~K} / \mathrm{Gyr} \frac{\dot{\alpha}}{H_{0} \alpha} \Delta t .
\end{aligned}
$$

En concordancia con la referencia Labrosse y Jaupart [82], el enfriamiento total 
experimentado por la Tierra luego de la fase inicial del magma oceánico no puede superar los $200 \mathrm{~K}$; así, en los últimos 2,5 Gyr, $\Delta T_{m}<200 \mathrm{~K}$. A partir de estas restricciones, podemos obtener un límite para el cambio temporal de $\alpha$,

$$
\left|\frac{\dot{\alpha}}{H_{0} \alpha}\right|_{0}<1,93 \times 10^{-6} .
$$

Insertando este resultado en la ec.(2.22), vemos que,

$$
\left(\frac{\ell_{B}}{\ell_{P}}\right)^{2}<0,15, \quad \frac{\ell_{B}}{\ell_{P}}<0,39 .
$$

Por otro lado, se puede obtener otra cota diferente mediante la siguiente observación: La potencia radiada total de la Tierra $Q_{\text {tot }}$ se puede explicar por la desintegración radiactiva de un plazo de veinte por ciento [82]. Los datos más recientes se estimaron a partir de un ajuste de 38.347 mediciones. La metodología consistió en utilizar una aproximación de la refrigeración media para la circulación hidrotermal en la corteza oceánica joven; y en el resto de la superficie de la Tierra, el flujo de calor promedio de varios dominios geológicos se estimó según la definición global de mapas digitales provistos por la geología, y luego un estimación global que se obtiene multiplicando la superficie total mundial de los dominios geológicos, [39].

Estos resultados muestran que $Q_{t o t} \approx 47 \mathrm{TW}$ (en la Tabla 4.3 esta estimación se encuentra separada en las contribuciones continental y oceánica). Por lo tanto,

$$
\left|Q_{\text {mach }}\right|=\left|M_{E} C_{P} \lambda_{G} T_{m}(t)\right|<0,2 Q_{\text {tot }} .
$$

\begin{tabular}{|c|c|c|c|}
\hline Parte de la Tierra & Area $\left(10^{14} \mathrm{~m}^{2}\right)$ & $\mathrm{FC}(\mathrm{TW})$ & $\mathrm{PFC}\left(\frac{\mathrm{mW}}{\mathrm{m}^{2}}\right)$ \\
\hline Continente & 2.073 & 14.7 & 70.9 \\
\hline Océano & 3.028 & 31.9 & 105.4 \\
\hline Global Total & 5.101 & 46.7 & 91.6 \\
\hline
\end{tabular}

Cuadro 4.3: Resumen de los flujos de calor de las estimaciones de [39], FC flujo de calor, PFC principal flujo de calor 
Luego en un intervalo de 2,5 Gyr, hallamos que,

$$
\left|\frac{\dot{\alpha}}{H_{0} \alpha}\right|_{0}<3,98 \times 10^{-6},
$$

y

$$
\left(\frac{\ell_{B}}{\ell_{P}}\right)^{2}<0,31, \quad \frac{\ell_{B}}{\ell_{P}}<0,55 .
$$

\subsubsection{Flujos de calor de planetas exteriores}

A Júpiter, Saturno, Urano y Neptuno se los llama frecuentemente planetas gaseosos ya que son planetas muy masivos con una gruesa atmósfera gaseosa y un núcleo sólido. Los dos primeros están compuestos principalmente por Hidrógeno y Helio, mientras que los últimos son denominados también planetas helados porque su composición es esencialmente de hielos de Agua, Amoníaco y Metano. De la comparación entre las temperaturas bolométricas observadas de los planetas gigantes con los valores esperados cuando estos planetas están en equilibrio térmico con la radiación solar incidente, está claro que todos ellos a excepción de Urano tienen una importante fuente interna de calor [69].

En el caso de Júpiter, este residuo de calor primordial es causado por el enfriamiento continuo y el encogimiento del planeta via el mecanismo de Kelvin-Helmholtz.

Saturno también debe haber comenzado con un interior caliente como Júpiter, dado que sus formaciones fueron similares. Pero por ser un poco más pequeño y menos masivo, Saturno no fue tan caliente en el inicio de su vida y ha tenido tiempo de enfriarse. Como resultado, el planeta ha perdido la mayor parte de su calor primordial debiendo existir otra fuente que contribuya con la mayor parte de su calor interno. Este exceso de calor se genera por la precipitación de Helio en su núcleo de Hidrógeno metálico. Cuanto más pesado es el Helio más se separa del Hidrógeno que es más ligero, y cae hacia el centro. Pequeñas gotas de Helio se forman donde esté lo suficientemente frío, precipitándose hacia abajo, y luego se disuelven en los niveles calientes más profundos. Como el Helio a altos niveles forma una "lluvia" a través de los alrededores del Hidrógeno, el Helio convierte parte de su energía en calor, [90].

No se conoce mucho acerca del bajo valor del calor interno de Urano. Una hipótesis es que los gradientes de su composición química pueden actuar como in- 
hibidores del transporte de calor desde el interior caliente a la superficie. Otros sugieren que el planeta fue golpeado por un impactador supermasivo que provocó la expulsión de la mayor parte de su calor primordial, dejándolo con una temperatura interna agotada. Urano tiene como mucho $4 M_{\oplus}$ de materiales rocosos, por tanto, parte del flujo interno $\left(\approx 0,02 \mathrm{Wm}^{-2}\right)$ proviene de la desintegración radiactiva, aunque no se descarta la acción del mecanismo de Kelvin-Helmholtz.

Si bien Neptuno se encuentra a una distancia mayor del Sol que Urano su emisión termal es casi equivalente. Se han propuesto varias posibles explicaciones las cuales incluyen: el calentamiento radiogénico de su núcleo, la conversión de metano a alta presión en los hidrocarburos de Hidrógeno, diamantes y más complejos hidrocarburos (el hidrógeno y el diamante entonces se levantaría y se hundiría, respectivamente, produciendo liberación de energía potencial gravitatoria), y la convección en la atmósfera más baja que hace que las ondas de gravedad se rompan por encima de la tropopausa, [57, 68].

Calculamos los flujos $J_{\zeta_{i}}$ para cada planeta mediante la ecuación para la conducción de calor

$$
\frac{1}{r^{2}} \frac{d}{d r}\left(K r^{2} \frac{d T}{d r}\right)=-\varepsilon \rho,
$$

siendo $K$ la conductividad termal efectiva del material planetario. El flujo de calor es,

$$
\mathbf{J}=-K \frac{d T}{d r}
$$

$\mathrm{Si} \bar{\varepsilon}$ es la principal producción de calor, que se estima de los resultados de la Tabla 4.1 de acuerdo con la composición química de cada planeta, luego

$$
J(r)=-K \frac{d T}{d r}=\frac{1}{r^{2}} \int_{0}^{\infty} \varepsilon\left(r^{\prime}\right) \rho\left(r^{\prime}\right) d r^{\prime}=\bar{\varepsilon} \frac{m(r)}{4 \pi r^{2}} .
$$

Así el flujo superficial es,

$$
\mathbf{J}_{\zeta_{i}}=-\left.K \frac{d T}{d r}\right|_{\zeta_{i}}=\bar{\varepsilon} \frac{m\left(R_{i}\right)}{4 \pi R_{i}^{2}},
$$

que es la ecuación fundamental de todo este desarrollo. Más tarde cuando comparamos los resultados para $J_{\zeta_{i}}$ con los flujos observados por las misiones Voyager 1, 2, 
and Cassini, [110], obtenemos la Tabla 4.4.

\begin{tabular}{|c|c|c|c|c|}
\hline Planeta & $J_{\text {obs }}\left(\mathrm{W} / \mathrm{m}^{2}\right)$ & $\mathrm{M}(\mathrm{Kg})$ & $\mathrm{R}(\mathrm{m})$ & $J_{\zeta_{i}}\left(\mathrm{~W} / \mathrm{m}^{2}\right) \frac{\dot{\alpha}}{H_{0} \alpha}$ \\
\hline Jupiter & $5,44 \pm 0,43$ & $1,90 \times 10^{27}$ & $7,14 \times 10^{7}$ & $6,35 \times 10^{4}$ \\
\hline Saturno & $2,01 \pm 0,14$ & $5,68 \times 10^{26}$ & $6,03 \times 10^{7}$ & $2,71 \times 10^{4}$ \\
\hline Urano & $0,042 \pm 0,047$ & $8,68 \times 10^{25}$ & $2,556 \times 10^{7}$ & $2,08 \times 10^{4}$ \\
\hline Neptuno & $0,43 \pm 0,09$ & $1,02 \times 10^{26}$ & $2,47 \times 10^{7}$ & $3,44 \times 10^{4}$ \\
\hline
\end{tabular}

Cuadro 4.4: Los flujos de calor observados, las masas, los radios, y los flujos de calor calculados de los planetas exteriores

Los límites superiores a " $3 \sigma$ ", y las correspondientes cotas a " $\left(\ell_{B} / \ell_{P}\right)$ " que obtenemos son los presentados en la tabla 4.5,

\begin{tabular}{|l|c|c|}
\hline Planeta & $\frac{\dot{\alpha}}{H_{0} \alpha} \mid$ & $\left(\ell_{B} / \ell_{P}\right)$ \\
\hline Jupiter & $2,04 \times 10^{-5}$ & 1,25 \\
Saturno & $1,55 \times 10^{-5}$ & 1,09 \\
Urano & $6,75 \times 10^{-6}$ & 0,72 \\
Neptuno & $7,85 \times 10^{-6}$ & 0,78 \\
Tierra $^{(1)}$ & $1,93 \times 10^{-6}$ & 0,39 \\
Tierra $^{(2)}$ & $3,98 \times 10^{-6}$ & 0,55 \\
\hline
\end{tabular}

Cuadro 4.5: Cotas para los planetas exteriores y la Tierra ( ${ }^{(1)}$ resultados de las ecs.(4.31) y (4.32) mientras que ${ }^{(2)}$ son de las ecs.(4.34) y (4.35)).

\subsection{Resultados}

La mejor cota encontrada en esta tesis para la variación temporal de $\alpha$ es la que se obtuvo mediante el análisis de los aspectos geotérmicos de la Tierra, que son naturalmente, los más claramente entendidos y fiables de nuestro sistema solar y, el flujo de calor de la superficie es muy bajo. Nuestros límites son comparables con los que se obtuvieron mediante mediciones en laboratorios combinando las frecuencias de $\mathrm{Sr}$ (Blatt et al. [18]), Hg+ (Fortier et al. [56]), Yb+ (Peik et al. [111]) y H (Fischer 
et al. [55]) con las del Cesio [93, 135]. Además, son sólo un orden de magnitud más débil que la cota hallada gracias a los resultados de Oklo, que es el límite más estricto para la variación temporal de $\alpha$ hasta hoy $[58,135])$; y otra encontrada a partir de mediciones de la razón entre las frecuencias de $\mathrm{Al}+$ con $\mathrm{Hg}+$ en relojes ópticos en el período de un año [93, 120](ver la Tabla 4.6). Las cotas que encontramos dependen del modelo de enfriamiento para la Tierra, pero no hay un acuerdo general acerca de los mecanismos que existen detrás de él [71]. Los planetas exteriores nos han proporcionado con restricciones adicionales, que están entre el mismo y/o un orden de magnitud más débiles que la de la Tierra.

\begin{tabular}{|c|c|c|}
\hline Observación & $\left|\frac{\dot{\alpha}}{\alpha}\right|\left(\mathrm{yr}^{-1}\right)$ & Referencia \\
\hline RelojCs & $(3,3 \pm 3,0) \times 10^{-16}$ & $(1)$ \\
RelojHg & $(5,3 \pm 7,9) \times 10^{-17}$ & $(1)$ \\
Oklo & $(2,50 \pm 0,83) \times 10^{-17}$ & $(2)$ \\
$\mathrm{J}_{\oplus}$ & $1,52 \times 10^{-16}$ & $(3)$ \\
$\mathrm{J}_{\oplus} I I$ & $3,14 \times 10^{-16}$ & $(4)$ \\
$\mathrm{J}_{\mathrm{Jup}}$ & $1,61 \times 10^{-15}$ & $(5)$ \\
$\mathrm{J}_{\mathrm{Sat}}$ & $1,22 \times 10^{-15}$ & $(5)$ \\
$\mathrm{J}_{\mathrm{Ur}}$ & $5,32 \times 10^{-16}$ & $(5)$ \\
$\mathrm{J}_{\mathrm{Nep}}$ & $6,19 \times 10^{-16}$ & $(5)$ \\
\hline
\end{tabular}

Cuadro 4.6: Tabla que muestra los diferentes tipos de cotas, el valor de $\frac{\dot{\alpha}}{\alpha_{0}}$, y las referencias. Referencias (1) Li et al. [93]; (2)Fujii et al. [58]; (3) Ec.4.31; (4) Ec.4.34; (5) Tabla4.5 


\section{Capítulo 5}

\section{Límites para las interacciones fundamentales y acoplamientos}

En este capítulo analizaremos la relación entre las interacciones fundamentales electromagnética, fuerte y débil con el Principio de Equivalencia Débil (WEP) de manera tal de encontrar cotas a los parámetros de violación a dicho principio. Luego, estudiaremos las variaciones espaciales de las constantes fundamentales $\alpha, \lambda_{Q C D}$, $\sin ^{2} \theta_{W}$ y $G_{F}$, sus conexiones con las fuerzas mencionadas anteriormente y las cotas alcanzadas a la hora de ser testeadas con experimentos de Eötvös. Además, acotaremos las variaciones espaciales de las constantes fundamentales de Gauge $\alpha_{1}, \alpha_{2}, \alpha_{3}$, el valor de expectación del Higgs $v$ y de las masas de partículas fundamentales gracias al vínculo que existe entre todos estos parámetros y las constante fundamentales elegidas en este caso.

\subsection{El Principio de Equivalencia y los experimen- tos Eötvös}

El Principio de Equivalencia es considerado el pilar fundamental en el desarrollo de la teoría de la gravitación. Tanto la mecánica newtoniana clásica así como la de Einstein, la Relatividad General, se basan en esta fuerte afirmación. El "Principio de Equivalencia Débil" (WEP) afirma que la "masa inercial" $m_{I}$ (que regula la respuesta de un objeto a un fuerza aplicada), debe ser igual a la "masa peso" o "masa pasiva" 
$m_{P}$, que se encuentra relacionada con la respuesta a la interacción gravitatoria. Una alternativa a este enunciado es la "Universalidad de la Caída Libre" (UFF), que dice que la línea de Universo de un cuerpo en caída libre en un caso donde sólo se considera la interacción gravitatoria, es independiente de su composición y de su estructura. Más potente a estas declaraciones es el "Principio de Equivalencia de Einstein" (EEP), agrupando al WEP, y a las Invariancias Locales de Lorentz (LLI) y de Posición (LPI). Por lo tanto, el resultado de cualquier experimento local no gravitatorio es independiente del lugar y del tiempo en el Universo donde se lleve a cabo, así como también lo es el valor de la velocidad en caída libre del marco de referencia. De acuerdo con esto, si el EEP es válido, entonces el efecto de la gravedad causa la curvatura del espacio-tiempo y se cumplen los siguientes supuestos:

- la métrica del espacio-tiempo es simétrica;

- las trayectorias de los cuerpos en caída libre están conformadas por las geodésicas de la métrica;

- en marcos de referencia locales libres, las leyes no gravitatorias de la física son aquellas que compatibilizan con la Relatividad Especial como por ej. las ecuaciones de Maxwell;

siendo necesaria para ello la constancia de varios parámetros fundamentales, como ya mencionamos en la introducción, que aparecen en el Modelo Estándar para describir los fenómenos de la naturaleza, las constantes fundamentales [145].

Una forma sencilla de detectar cualquier ruptura del WEP es comparar las aceleraciones de dos cuerpos de laboratorio de diferente composición inmersos en un campo gravitatorio externo. Suponiendo que $m_{I}$ y $m_{P}$ se diferencian entre sí, la aceleración del cuerpo estará impartida por la relación $m_{I} a=m_{P} g$, donde $g$ es el campo gravitatorio local, dado que la masa inercial tiene contribuciones de varios tipos "energías" como la electromagnética, fuerte, etc. Este principio ha sido probado con gran precisión a partir de experimentos tipo Eötvös ([75, 119, 130] y otros), a través de los cuales se trata de medir la diferencia entre dos masas de diferentes materiales por el parámetro,

$$
\eta_{(A, B)}=\frac{\left|\boldsymbol{a}_{\boldsymbol{A}}-\boldsymbol{a}_{\boldsymbol{B}}\right|}{|\boldsymbol{g}|} .
$$


Consecuentemente, si alguna de estas energías llegase a contribuir de manera distintas a las masas, causaría una violación del WEP tal que,

$$
m_{P}-m_{I}=\delta m=-\sum_{t} \Gamma_{t} E^{t} / c^{2}
$$

donde $E^{t}$ representa la energía de enlace nuclear generada por la interacción $t$ y $\Gamma_{t}$ es un parámetro fenomenológico específico para cada tipo de interacción que mide la intensidad de la violación. Por lo general, estos parámetros se obtienen con al menos tres diferentes datos procedentes de los experimentos tipo Eötvös mediante un ajuste estadístico. También se pueden predecir a partir de algunas teorías de la gravitación, siendo entonces estos resultados una sensible prueba de esas últimas teorías. Por lo tanto, estos $\eta_{i}$ imponen límites superiores a los $\Gamma_{t}[32,145]$, y también se pueden utilizar para optimizar la elección de los materiales para los experimentos [33] (para un análisis profundo, acudir a las referencias $[22,136,145])$.

Por otro lado, Dicke (para más detalles ver $[42,64,134,135]$ ) concluyó que una variación espacial de cualquiera de las constantes de acoplamiento podría causar una violación de la UFF. Esto se debe a un cuerpo compuesto como, por ejemplo un núcleo, está formado por partículas elementales (así, dependerá de los acoplamientos de Yukawa y de los parámetros del sector de Higgs), pero también, como se mencionó anteriormente, cuenta con contribuciones de las energías de ligadura que vienen de las interacciones. Entonces, la masa es una función complicada de los parámetros fundamentales $\alpha_{j}, m\left[\alpha_{j}\right]$. La acción de una partícula puntual $A$ es,

$$
S_{\text {matter }}=-\int m_{A}\left[\alpha_{j}\right] c \sqrt{-g_{\mu \nu}(\boldsymbol{x}) v^{\mu} v^{\nu}} d^{4} x
$$

En el límite newtoniano, a primer order de $v / c$ y suponiendo que las velocidades y variaciones son pequeñas, la ecuación de movimiento se reduce a,

$$
\begin{gathered}
\boldsymbol{a}=\boldsymbol{g}_{\boldsymbol{N}}+\delta \boldsymbol{a}_{\boldsymbol{A}}, \\
\delta \boldsymbol{a}_{\boldsymbol{A}}=-\sum_{j} \frac{c^{2}}{m_{A}} \frac{\partial m_{A}}{\partial \alpha_{j}} \nabla \alpha_{j} .
\end{gathered}
$$


Esta aceleración anómala aparece debido a los cambios en las energías de ligadura o enlace [42, 65, 107, 135], y también en los parámetros del sector de Higgs haciendo que las dependencias de las constantes fundamentales sean, a priori, dependendientes de la composición. Así, una variación de cualquiera de estas constantes de acoplamiento termina en una violación de la UFF de tal manera que, siendo la masa total del cuerpo espacio dependiente entonces, si la energía se conserva, una interacción anómala debe estar actuando. Las variaciones de las constantes fundamentales pueden ser limitadas con estas pruebas de validez del WEP [40].

Además de la contribución de las energías de enlace, hay contribuciones provenientes de la estructura interna de los nucleones [22], a través de la diferencia de masa $n-p$ que deben ser también tenidas en cuenta.

\subsection{Parámetros de violación $\Gamma$ a partir de las in- teracciones fundamentales}

En esta sección haremos un breve resumen del trabajo realizado por Chamoun y Vucetich [22] y luego vamos a hacer un ajuste de los parámetros $\Gamma_{t}$ adicionando nuevos datos experimentales $\eta_{(A, B)}$ a los que ya fueron utilizados en esta última referencia.

\subsubsection{Aportes de las diferencias de masa y de las energías de ligadura}

Hasta el momento en que Chamoun y Vucetich realizaron su trabajo [22], existían ya varios artículos sobre el estudio de los parámetros de violación a causa de las interacciones fundamentales $[136,145]$, pero ninguno de ellos tenía en cuenta los efectos de la contribuciones débiles de los nucleones individuales a la masa nuclear.

Hay un método desarrollado por Cottingham que permite la estimación de la diferencia de masa de neutrones y protones de las interacciones electromagnéticas, a partir de datos experimetales en una teoría de cuántica de campos, que también se ha generalizado a las interacciones fuertes. Utilizando dichas fórmulas, Chamoun y Vucetich construyeron un modelo independiente para la predicción de las contribu- 
ciones débiles para la diferencia de masa de neutrones y protones, que básicamente consiste en una regla de suma que describe a la masa libre de nucleones en términos de cantidades observables, siendo otra versión generalizada del método de Cottingham. Este estudio está basado en las referencias [22, 25, 31, 98].

A primer orden en la constante de estructura fina $\alpha$, la contribución electromagnética a la auto-energía del nucleón puede expresarse tal que,

$$
\Delta M_{N}^{E M}=\frac{i e^{2}}{2(2 \pi)^{4}} \int d^{4} q G_{E M}^{\mu \nu}\left(q^{2}\right) T_{\mu \nu}^{E M, N}\left(\boldsymbol{q}, q_{0}\right),
$$

donde $G_{E M}^{\mu \nu}\left(q^{2}\right)=\eta^{\mu \nu} / q^{2}$ es el propagador del fotón. $T_{\mu \nu}^{E M, N}\left(\boldsymbol{q}, q_{0}\right)$ describe la amplitud de scatteirng de Compton de un fotón virtual con momento $q$ por un nucleón $N$ en reposo. Esta magnitud puede reducirse mediante el uso de la aproximación de Born para luego, haciendo una rotación de Wick y un poco de álgebra transformar la ecuación (5.5) en,

$$
\begin{gathered}
f_{1}\left(q^{2}\right)=\frac{\alpha}{\pi} \frac{G_{M}^{2}\left(q^{2}\right)-G_{E}^{2}\left(q^{2}\right)}{q^{2}+4 M^{2}}, \\
f_{2}\left(q^{2}\right)=\frac{\alpha}{\pi} \frac{q^{2} G_{M}^{2}\left(q^{2}\right)+4 M^{2} G_{E}^{2}\left(q^{2}\right)}{q^{2}\left(q^{2}+4 M^{2}\right)}, \\
\Delta M_{N}^{E M}=-\frac{1}{\pi} \int_{0}^{\infty} \frac{q d q}{q^{2}} \int_{0}^{q} d \nu \sqrt{q^{2}-\nu^{2}} \frac{4 M q^{2}}{q^{4}+4 M^{2} \nu^{2}} \\
\times\left[3 q^{2} f_{1}\left(q^{2}\right)-\left(q^{2}+2 \nu^{2}\right) f_{2}\left(q^{2}\right)\right],
\end{gathered}
$$

donde las expresiones $f_{1}\left(q^{2}\right)$ y $f_{2}\left(q^{2}\right)$ son determinadas gracias a los factores de Sachs electromagnéticos del nucleón $G_{E, M}^{N}$, que se pueden medir a partir de datos de dispersión electrón-nucleón (ver $[4,60,106,112]$ ), tomando además $M=938 \mathrm{MeV}$. La contribución electromagnética a la diferencia de masa del neutrón-protón se obtiene restando las dos masas propias (auto-masas) de las partículas $\Delta M_{n p}^{e m}=$ $\Delta M_{n}^{e m}-\Delta M_{p}^{e m}=-0,79 \mathrm{MeV}$ (midiendo las masas en unidades $\mathrm{MeV}$ ).

Del mismo modo, es posible encontrar la contribución fuerte a la diferencia de masa del neutrón-protón (debido a la diferencia de masa entre los quarks up y down [99, 108]) utilizando diversos métodos, como modelos de mezcla $\rho-\omega$, modelos Skyrme ,modelos quirales soltónicos y modelos Sigma [25, 29, 50, 54, 62, 70, 109] llegando a $\Delta M_{n p}^{s t}=\Delta M_{n}^{s t}-\Delta M_{p}^{s t}=2,08 \mathrm{MeV}$. 
Estos resultados de las contribuciones electromagnética y fuerte son válidos en la fórmula Cottingham al orden más bajo en $\alpha$, mientras que las correcciones de orden superior deben ser renormalizadas. Sin embargo, las correcciones a la diferencia de masa que depende del punto de renormalización $\mu$ son muy pequeñas por lo que pueden ser descartadas, hay una gran explicación sobre el tema en [62].

Con el fin de encontrar una generalización para la diferencia débil de masa entre neutrones y protones, se debe empezar a trabajar con la fórmula para la interacción de cuatro fermiones como una aproximación de bajas energías de la teoría IVB correspondiente al intercambio de los bosones, $\mathcal{L}^{\text {eff }}{ }^{1}$ que es,

$$
\begin{aligned}
\mathcal{L}^{e f f} & =\mathcal{L}_{c c}^{e f f}+\mathcal{L}_{n c}^{e f f} \\
& =\frac{-g^{2}}{2 M_{W}^{2}} J_{\mu}^{+} J^{-\mu}+\frac{-g^{2}}{2 M_{W}^{2}} J_{\mu}^{N} J^{N \mu}, \\
J_{\mu}^{+} & =J_{\mu}^{+V}-J_{\mu}^{+A} \\
& =\frac{1}{2} \sum_{f=\nu, e, u, d} \bar{f} \gamma_{\mu}\left(1-\gamma_{5}\right) T^{-} f, \\
J_{\mu}^{-} & =\left(J_{\mu}^{+}\right)^{\text {dagger }},
\end{aligned}
$$

siendo la ecuación de arriba la corriente de carga de una familia de fermiones; y

$$
\begin{gathered}
J_{\mu}^{N}=\frac{1}{2} \sum_{f=\nu, e, u, d}\left[\bar{f} \gamma_{\mu}\left(T_{3}-2 Q \sin ^{2} \theta_{W}\right) f\right. \\
\left.-\bar{f} \gamma_{\mu} \gamma_{5} T_{3} f\right], \\
J_{\mu}^{N}=J_{\mu}^{N V}-J_{\mu}^{N A},
\end{gathered}
$$

representa la corriente neutral donde $Q$ es la matriz de carga y $T_{i}=\sigma_{i} / 2$ la matriz de los generadores del álgebra $S U(2), T^{ \pm}=T_{1} \pm i T_{2}$. La interacción débil podría contribuir en un término del hamiltoniano del nucleón, por lo que es necesario calcular la diferencia entre los elementos de la matriz de neutrones y de protones del operador $H$. Ni las corrientes de carga ni la parte axial de la corriente neutral contribuirán a

\footnotetext{
${ }^{1}$ esta aproximación de las interacciones puramente hadrónicas tiene grandes correcciones en QCD, que se pueden estimar (ver [113]) y teniendo en cuenta estos efectos, se introduce el factor $\mathcal{G} \approx 8$
} 
la diferencia de masas $n-p$ y sólo el vector neutro de corriente de lo hará. Afortunadamente, este último tiene la misma estructura que la corriente electromagnética, por lo tanto, el tratamiento es similar a la que hecho antes. Entonces,

$$
\begin{gathered}
\Delta M_{N}^{W}=-\frac{1}{\pi} \int_{0}^{\infty} \frac{q d q}{q^{2}} \int_{0}^{q} d \nu \sqrt{q^{2}-\nu^{2}} \frac{4 M q^{2}}{q^{4}+4 M^{2} \nu^{2}} \\
\times\left[3 q^{2} f_{1}\left(q^{2}\right)-\left(q^{2}+2 \nu^{2}\right) f_{2}\left(q^{2}\right)\right], \\
f_{1}\left(q^{2}\right)=\frac{\alpha_{W}}{\pi} \frac{\left[G_{M}^{Z}\left(q^{2}\right)\right]^{2}-\left[G_{E}^{Z}\left(q^{2}\right)\right]^{2}}{q^{2}+4 M^{2}} \\
f_{2}\left(q^{2}\right)=\frac{\alpha_{W}}{\pi} \frac{q^{2}\left[G_{M}^{Z}\left(q^{2}\right)\right]^{2}+4 M^{2}\left[G_{E}^{Z}\left(q^{2}\right)\right]^{2}}{q^{2}\left(q^{2}+4 M^{2}\right)} \\
\alpha_{W}=\frac{\sqrt{2} G_{F} M^{2}}{\pi}=0,463 \times 10^{-5} .
\end{gathered}
$$

Los factores de Sachs débiles, no se han medido, a excepción de puntos aislados [97], pero pueden estar relacionados con los electromagnéticos [102]. Así, el resultado final es de $\Delta M_{n p}^{W}=\Delta M_{n}^{W}-\Delta M_{p}^{W}=-4,7 \mathrm{eV}$ y la incerteza, $0,9 \mathrm{eV}$, fue estimado a partir de las diferencias entre la parametrización Galster y experimentos.

Las energías de ligadura fuerte y electromagnética se pueden calcular con la fórmula semi-empírica de masas [95] mientras que la energía débil está estudiada en detalle en el artículo de Haugan y Will [65].

$$
\begin{gathered}
E_{b}^{e m}=-\frac{a_{c} Z(Z-1)}{A^{1 / 3}}, \\
E_{b}^{s t}=a_{v} A-a_{s} A^{2 / 3}-a_{A} \frac{(A-2 Z)^{2}}{A}+\cdots, \\
E_{b}^{W}=G_{F} 2^{-2 / 3} V^{-1}\left\{N Z\left[\left(3 \alpha_{\beta}^{2}-1\right)+4 A_{n} A_{p}\right]\right. \\
\left.+\frac{N^{2}}{2} A_{n}^{2}\left(1+3 a_{n}^{2}\right)+\frac{Z^{2}}{2} A_{p}^{2}\left(1+3 a_{p}^{2}\right)+\cdots\right\},
\end{gathered}
$$

con $a_{c} \sim \frac{3}{5}\left(\frac{\hbar c \alpha}{r_{0}}\right) ; \alpha_{\beta} \sim 1,21$ que está impuesta por las mediciones de los decaimientos $\beta$, y $V=5,13 \times 10^{-39} A \mathrm{~cm}^{3}$. $a_{v}, a_{s}$ y $a_{A}$ son los coeficientes de volumen, surperficie y asimetría respectivamente $\left\{a_{v} / M, a_{s} / M, a_{A} / M\right\} \approx\{15,7,17,8,23,7\}$. Estos términos podrían a priori acoplarse con la constante fuerte $\alpha_{S}$ que se encuentra relacionada 
con $\Lambda_{Q C D}$. Además,

$$
\begin{gathered}
A_{n}=A_{s}-A_{v}, \quad a_{n}=\left(A_{s} a_{s}^{1}-A_{v} a_{v}^{1}\right) / A_{n}, \\
A_{p}=A_{s}+A_{v}, \quad a_{p}=\left(A_{s} a_{s}^{1}+A_{v} a_{v}^{1}\right) / A_{p}, \\
A_{v}=\frac{1}{2}\left(1+2 A_{s}\right), \quad A_{s}=-\sin ^{2} \theta_{W}, \quad a_{v}^{1}=\left(1+2 A_{s}\right)^{-1}, \quad a_{s}^{1}=0 .
\end{gathered}
$$

Los experimentos tipo Eötvös como ya se mencionó en capítulos y secciones anteriores, miden la diferencia en la aceleración $a$ de cuerpos diferentes $A$ y $B$ en caída libre inmersos en un campo gravitatorio $g$, imponiendo cotas superiores en la diferencia entre $m_{P}$ and $m_{I}\left(\delta m=m_{P}-m_{I}\right)$. Siendo $m(Z, N)$ la masa nuclear, $E_{b}$ la energía de ligadura; los parámetros de Eötvös $\eta_{(A, B)}$ se definen como,

$$
\begin{gathered}
m(Z, N)=Z M^{p}+N M^{n}-E_{b}, \\
\eta_{(A, B)}=\frac{a_{A}-a_{B}}{g}=\left[\left(\frac{\delta m}{m_{I}}\right)_{A}-\left(\frac{\delta m}{m_{I}}\right)_{B}\right] .
\end{gathered}
$$

Tomando un par de aproximaciones, como pequeños cambios en las energías de enlace y la invarianza de $\delta \bar{M}, M^{n}$ y $M^{p}$ a través de la tabla periódica, dicho parámetro puede expresarse,

$$
\begin{aligned}
\eta_{(A, B)} & =\left(\frac{\sum_{t=s t, W, e m} \Gamma_{t}\left[\left(\frac{N-Z}{2}\right)\left(\Delta M_{n-p}\right)^{t}-E^{t}\right]}{m}\right)_{A} \\
& -\left(\frac{\sum_{t=s t, W, e m} \Gamma_{t}\left[\left(\frac{N-Z}{2}\right)\left(\Delta M_{n-p}\right)^{t}-E^{t}\right]}{m}\right)_{B}
\end{aligned}
$$

\subsubsection{Contraste entre la teoría y las observaciones}

Una vez que fueron calculadas las contribuciones de las diferencias de masas $n-p$ y las energías de enlace, es posible hacer una comparación entre los resultados teóricos con los parámetros experimentales $\eta_{(A, B)}$ (ver los primeros seis datos de la Tabla 5.1) y establecer límites a los $\Gamma_{t}$ mediante un ajuste numérico (los resultados están en la Tabla 5.2). 
Cuadro 5.1: Resultados de los experimentos Eötvös $(1 \sigma)$.

\begin{tabular}{cccc}
\hline$A$ & $B$ & $\eta(A, B) \times 10^{11}$ & Ref. \\
\hline $\mathrm{Al}$ & $\mathrm{Au}$ & $1,0 \pm 1,5$ & {$[119]$} \\
$\mathrm{Al}$ & $\mathrm{Pt}$ & $-0,03 \pm 0,07$ & {$[19]$} \\
$\mathrm{Cu}$ & $\mathrm{W}$ & $0,6 \pm 2,0$ & {$[75]$} \\
$\mathrm{Be}$ & $\mathrm{Al}$ & $-0,02 \pm 0,28$ & {$[130]$} \\
$\mathrm{Be}$ & $\mathrm{Cu}$ & $-0,19 \pm 0,25$ & {$[1]$} \\
$\mathrm{Si} / \mathrm{Al}$ & $\mathrm{Cu}$ & $0,51 \pm 0,67$ & {$[130]$} \\
\hline Tierra & Luna & $-0,01 \pm 0,014$ & {$[146]$} \\
Roca terrestre & Roca lunar & $0,04 \pm 0,11$ & {$[8]$} \\
$\mathrm{Be}$ & $\mathrm{Ti}$ & $0,003 \pm 0,018$ & {$[122]$} \\
$\mathrm{Be}$ & $\mathrm{Al}$ & $-0,015 \pm 0,015$ & {$[2]$} \\
\hline
\end{tabular}

Cuadro 5.2: Cotas superiores para los parámetros de violación de la UFF. La 1er columna representa los resultados suponiendo que una única interacción rompe el WEP, y la 2da representa los resultados suponiendo que todas las 3 interacciones violan al principio.

\begin{tabular}{ccc}
\hline Par & $\Gamma_{t} \mathrm{c} / 1$ int. & $\Gamma_{t} \mathrm{c} / 3$ int. \\
\hline$\Gamma_{e m} \times 10^{9}$ & 1,1 & 9,6 \\
$\Gamma_{s t} \times 10^{9}$ & 1,2 & 14. \\
$\Gamma_{W} \times 10^{3}$ & 1,0 & 33. \\
\hline
\end{tabular}


Cuadro 5.3: Cotas superiores para los "nuevos" parámetros de violación UFF. La 1er columna representa los resultados suponiendo que una sola interacción no cumple el WEP, y la 2da representa a los resultados suponiendo que las tres interacciones rompen el WEP.

\begin{tabular}{ccc}
\hline Par & $\Gamma_{t} \mathrm{c} / 1$ int. & $\Gamma_{t} \mathrm{c} / 3$ int. \\
\hline$\Gamma_{e m} \times 10^{9}$ & $0,002 \pm 0,069$ & $-0,12 \pm 6,69$ \\
$\Gamma_{s t} \times 10^{9}$ & $-0,009 \pm 0,023$ & $-0,02 \pm 4,75$ \\
$\Gamma_{W} \times 10^{3}$ & $0,126 \pm 0,142$ & $0,20 \pm 4,76$ \\
\hline
\end{tabular}

\subsubsection{Refinamiento de los parámetros $\Gamma$}

Desde 2002 hasta la actualidad se han llevado a cabo varios experimentos tipo Eötvös pudiéndose alcanzar mejores precisiones y esto lleva a que los límites presentados en la referencia [22] puedan ser mejorados. Por eso nosotros recalculamos los parámetros de violación $\Gamma_{t}$ agregando nuevos datos experimentales, pudiendo de esta manera mejorar las cotas superiores ya establecidas. Además queremos analizar la matriz de correlación de los parámetros.

Así, siguiendo los pasos realizados por [22] y explicados en la subsección anterior; agregamos los últimos cuatro datos de la Tabla 5.1 para encontrar las restricciones que se muestran en la Tabla 5.3, que son uno o dos órdenes (dependiendo la fuerza) de magnitud más rigurosas que las establecidas en [22].

La matriz de correlación suponiendo que las tres interacciones violan al WEP es,

$$
\left.\operatorname{Corr}\right|_{\Gamma_{t=e m, s t, W}}=\left(\begin{array}{lcl}
1 & -0,95 & 0,72 \\
-0,95 & 1 & -0,88 \\
0,72 & -0,88 & 1
\end{array}\right)
$$

Analizándola podemos observar que los parámetros $\Gamma_{t=e m, s t, W}$ están áltamente correlacionados entre sí (la correlación más baja es 0.72 entre las fuerzas electromagnética y débil). Como resultado de esto, no deben ser analizadas por separado. Esta última deducción nos lleva a pensar que estas violaciones pueden existir y solaparse entre ellas dificultando su estudio. Entonces, es muy importante ser muy cuidadosos a la hora de considerar las violaciones al Principio de Equivalencia Débil causadas por las interacciones fundamentales. 


\subsection{Límites para las variaciones espaciales de las constantes fundamentales}

Si las constantes fundamentales pudieran variar en el Universo, entonces dichos cambios deberían darse de manera tal que alcancen los valores necesarios para que se pueda desarrollar la "vida" como la conocemos. Dicke [41, 42, 64, 135] demostró que los gradientes espaciales de las constantes fundamentales pueden llegar a producir términos anómalos en las aceleraciones y de esta manera violar la UFF. La expresión para la anomalía está dada por la ec. (5.4b). Por lo tanto, para poder testearla con los datos experimentales tenemos,

$$
\eta_{(A, B)}=\sum_{j} \frac{c^{2}}{g m_{A}} \frac{\partial m_{A}}{\partial \alpha_{j}} \alpha_{j} \frac{\nabla \alpha_{j}}{\alpha_{j}}-\sum_{j} \frac{c^{2}}{g m_{B}} \frac{\partial m_{B}}{\partial \alpha_{j}} \alpha_{j} \frac{\nabla \alpha_{j}}{\alpha_{j}} .
$$

Dado que las contribuciones a la masa $m$ provienen de las diferencias de masa neutron-proton y las energías de ligadura, es importante analizar las relaciones que tienen entre ellas con las constantes fundamentales. En este caso, las constantes que analizaremos son: la constante de estructura fina $\alpha$, la energía de escala QCD $\Lambda_{Q C D}$, la constante de Fermi $G_{F}$, y el ángulo Weinberg $\sin ^{2} \theta_{W}$.

Una manera de describir los procesos hadrónicos a bajas energías es utilizando una Teoría Efectiva de Campos (EFT) como la Teoría Perturbativa Quiral (CPT). Serot, Walecka y otros científicos [59, 124, 125] han desarrollado esta teoría para poder obtener un Lagrangiano quiral efectivo para el núcleo partiendo de un modelo muy rasonable como lo es el $\sigma-\omega$ propuesto por Walecka [23, 137], el cual describe a la materia nuclear. (para un análisis exhaustivo ir a $[5,45,59,121,124,125]$ y otros).

\subsubsection{La Teoría Perturbativa Quiral}

Esta teoría (CPT) permite estudiar como ya mencionamos antes, la dinámica a bajas energías de la Cromodinámica Cuántica (QCD) debido a que ésta es no perturbativa a la escala de energía en la que estamos trabajando, siendo imposible utilizar algún método perturbativo para extraer información de la función de par- 
tición de QCD [14]. En el límite quiral, las masas de los quarks livianos tienden a cero $m_{s}=m_{d}=m_{u}=0$, mientras que los otros quarks $c, d, \cdots$ se los toma como infinitamente pesados por lo cual los grados de libertad associados a estas partículas se congelan y pueden despreciarse a bajas energías. Luego, QCD tiene un sólo parámetro que es invariante de escala en el grupo renormalizable llamado $\Lambda_{Q C D}$ [62]. Consecuentemente, se puede usar una teoría que sea invariante de escala [86, 129].

El Lagrangiano de una teoría invariante de escala no contiene una constante con unidades de masa; esto quiere decir que si una cantidad observable adimensional $\rho(Q)$ depende de una variable de "masa" $Q$, entonces la teoría tendrá una dependencia con $Q$ y $\rho(Q)$ no será independiente. Al efectuar un cambio de variables $Q^{\prime}=\lambda Q$, no se absorbe ninguna transformación dentro de $\rho(Q)$, por lo que dependerá del sistema de unidades elegido. Esto es una contradicción dado que una variable adimensional no puede depender del sistema de unidades y esto puede ilustrarse con el siguiente argumento dado por Stevenson [129]:

Teorema: Una función $f(x, y)$ que sólo depende de dos variables masivas $x$ e $y, y$ además cumple con las siguientes propiedades:

- ser adimensional,

- ser definida de manera única,

- cuya definición no incluye una constante masiva;

solamente puede ser función de $x / y$.

Corolario: $\operatorname{Si} f(x, y)$ es independente de $y, f(x, y)$ será constante.

El hecho es que en el Modelo Estandard, los Lagrangianos que se utilizan no definen una sino un grupo de infinitas teorías, quienes están todas parametrizadas por una constante de acoplamiento $g_{0}$ que toma valores en todo el eje real. Concluyendo, $\rho(Q)$ no está únicamente definida.

En QCD la primer derivada de $\rho(Q)$ es,

$$
\frac{d \rho}{d Q}=\frac{B(\rho)}{Q}
$$

$B(\rho)$ es una función adimensional con un argumento que también lo es y está definido 
de manera única por la teoría. Integrando y elegiendo $\infty$ por conveniencia,

$$
\ln Q-c t e=\int_{\infty}^{\rho} \frac{d \rho^{\prime}}{B\left(\rho^{\prime}\right)}=K(\rho)
$$

La forma más común de escribir la constante de integración es cte $=K_{0}-\ln \mu$, con $K_{0}=K(\rho(\mu))$ y $\mu$ representa un parámetro masivo arbitrario de la teoría. Suponiendo que existe una escala de energía $\Lambda$ para la cual $K(\rho(\mu))=0$, y elegiendo $\Lambda=\mu$ tal que $K_{0}=0$

$$
\rho(Q)=K^{-1}\left[\ln \left(\frac{Q}{\Lambda}\right)\right]
$$

o para un $\mu$ arbitrario,

$$
\rho(Q)=K^{-1}\left[K(\rho(\mu))-\ln \left(\frac{Q}{\mu}\right)\right] .
$$

Si bien $\Lambda$ y $\mu$ no se determinan a partir de la teoría, $\Lambda$ puede obtenerse experimentalmente pero no $\mu$. Haciendo una extensión del análisis dimensional de una cantidad medible, ésta es función de $\mathrm{n}$ variables masivas $x_{i}, \sigma\left(x_{1}, x_{2}, \cdots, x_{n}\right)$ con dimensión $M a s a^{D}$, y recordando que la segunda propiedad no se satisface:

$$
\sigma\left(x_{1}, x_{2}, \cdots, x_{n}\right)=x_{1}^{D} S\left(\frac{x_{2}}{x_{1}}, \cdots, \frac{x_{n}}{x_{1}} ; \frac{x_{1}}{\mu}, \rho(\mu)\right),
$$

$\mathrm{S}$ es independente de $\mu$. En el límite quiral, el único parámetro de dimensión relevante es $\Lambda_{Q C D}$. Por lo tanto, cualquier cantidad de dimensiones $M_{a s a^{D}}$ o Energia ${ }^{D}$ se puede describir como,

$$
\sigma=\Lambda_{Q C D}^{D} f\left[\frac{Q}{\Lambda_{Q C D}}\right]
$$

donde $f$ depende de la forma específica de la teoría hadrónica. Los observables adimensionales no dependen del tiempo a este límite. Las cantidades estáticas toman la siguiente forma,

$$
\sigma=\Lambda_{Q C D}^{D} X
$$

siendo $X$ una constante numérica definida por la teoría.

Nosotros queremos trabajar cerca del límite quiral para poder hacer un desarrollo de la masa del nucleón en potencias de la masa del pión, $m_{\pi}$ gracias a CPT [14, 62, 
117]. $m_{\pi}$ se encuentra relacionada con las masas de los quarks $u$ y $d$ (para las cuales tomamos su promedio, $\left.m_{q}=\left(m_{u}+m_{d}\right) / 2\right)$ via PCAC y la relación Gell-MannOakes-Renner $m_{\pi}^{2} f_{\pi}^{2}=-2 m_{q}<\bar{q} q>$; siendo $f_{\pi}$ la constante del decaimiento del pión, mientras que $\left\langle\bar{q} q>=\frac{1}{2}<\bar{u} u+\bar{d} d\right\rangle$ denota la condensación quiral. Ambos valores se toman en el límite quiral $\left(-\frac{<q \bar{q}>}{f_{\pi}^{2}} \sim \Lambda_{Q C D}\right.$, es el orden del parámetro de violación espontánea de simetría [30, 91, 148]). Los resultados del "lattice" QCD para describir a $m_{\pi}$ como función de la masa de los quarks $m_{q}$ son muy precisos y nos muestran que la principal relación lineal entre $m_{\pi}^{2}$ y $m_{q}$ permanece estable aún para grandes masas de quarks [117]. El siguiente resultado de $M_{N}\left(m_{\pi}\right)$ desarrollado en potencias de $m_{\pi}$ está dado por el cálculo a un "one-loop", teniendo en cuenta los diagramas hasta el orden quiral $p^{4}$ en la forma covariante $B \chi P T$ tal que,

$$
\begin{aligned}
M_{N}^{(4)} & =M_{0}-4 c_{1} m_{\pi}^{2}-\frac{3 g_{A}^{2}}{32 \pi f_{\pi}^{2}} m_{\pi}^{3} \\
& +\left[4 e_{1}^{(4)}(\lambda)-\frac{3}{64 \pi^{2} f_{\pi}^{2}}\left(\frac{g_{A}^{2}}{M_{0}}-\frac{c_{2}}{2}\right)\right. \\
& \left.-\frac{3}{32 \pi^{2} f_{\pi}^{2}}\left(\frac{g_{A}^{2}}{M_{0}}-8 c_{1}+c_{2}+4 c_{3}\right) \ln \frac{m_{\pi}}{\lambda}\right] m_{\pi}^{4} \\
& +\frac{3 g_{A}^{2}}{256 \pi f_{\pi}^{2} M_{0}^{2}} m_{\pi}^{5}+\mathcal{O}\left(m_{\pi}^{6}\right) .
\end{aligned}
$$

La constante de acoplamiento del vector axial del nucleón $g_{A}$ y la masa nuclear $M_{0}$ están tomadas en el límite quiral $\left(m_{\pi}=0\right) . c_{1}, c_{2}$ y $c_{3}$ son constantes a bajas energías. $c_{1}$ está estrechamente relacionada con el término pión-nucleón sigma, mientras que $c_{2}$ y $c_{3}$ comparten información primaria de la excitación $\Delta(1232)$ en la onda $\mathrm{P}$ de la dispersión pión-nucleón. Por último, $e_{1}^{(4)}(\lambda)$ representa la dinámica irresuelta a corta distancia. Este término compensa la dependencia logarítmica en la escala renormalizable de $\lambda$ a fin de garantizar la independencia del resultado de la escala (los detalles del desarrollo pueden ser vistos en $[62,116,117])$.

Sintetizando, trabajando en la vecindad del límite quiral, nos es posible expresar la masa del nucleón como función de $\Lambda_{Q C D}$ ya que, si bien la masa de los quarks no es nula, el término dominante es la masa $M_{0}$ que se desprende del límite quiral (proporcional a $\Lambda_{Q C D}$ ). En contraste a esto, la contribución fuerte a la diferencia de masa $n-p$ no depende de $\Lambda_{Q C D}$ pero sí de $G_{F}$. Esto ocurre debido a que la 
contribución del isospin fuerte se debe a la diferencia de masa de los quarks $u$ y $d$ que se determina por los acoplamientos de Yukawa en el SM de las interacciones electrodébiles y el valor de expectación en el vacío del campo de Higgs. Escribiendo $M_{p}$ como un desarrollo en serie y tomando los términos dominantes [108],

$$
\begin{aligned}
M_{p} & \approx M_{0}+(\bar{\alpha}+\bar{\beta}+2 \bar{\sigma}) m_{\pi}^{2}-\frac{1}{3}(2 \bar{\alpha}-\bar{\beta})\left(\frac{1-\eta_{m}}{1+\eta_{m}}\right) m_{\pi}^{2} \\
& -\frac{1}{8 \pi f^{2}}\left[\frac{3}{2} g_{A}^{2} m_{\pi}^{3}+\frac{4 g_{\Delta N}^{2}}{3 \pi} F_{\pi}\right],
\end{aligned}
$$

donde $\eta_{m}=m_{u} / m_{d}, \bar{\alpha}, \bar{\sigma}$ y $\bar{\beta}$ está relacionados con los contratérminos que entran en la expansión en serie al orden $\mathcal{O}\left(m_{q}\right)$, determinados por Beane et al. [108] como así también la función $F_{\pi} \Delta$-loop. El desarrollo de la masa del neutón se obtiene de manera similar al del protón intercambiando los quarks up y down. Luego,

$$
\Delta M_{n-p}^{s t}=M_{n}-\left.M_{p}\right|^{d-u} \approx \frac{2}{3}(2 \bar{\alpha}-\bar{\beta})\left(\frac{1-\eta_{m}}{1+\eta_{m}}\right) m_{\pi+}^{2} ;
$$

tal que $2 \bar{\alpha}-\bar{\beta} \propto \Lambda_{Q C D}^{-1}$; entonces llegamos a la conclusión que la diferencia de masa fuerte neutrón-protón es proporcional al cuadrado de la masa del pión $m_{\pi}$, que es a su vez proporcional al valor de expectación del Higgs $v$ (detalles en $[108,116,117]$ y más adelante); pero no depende de $\Lambda_{Q C D}$.

\subsubsection{Variaciones espaciales de las constantes fundamen- tales}

Podemos reescribir la ecuación (5.19) tal que,

$$
\begin{aligned}
\eta_{(A, B)} & =\left.\sum_{i} \frac{\partial\left(M_{n-p}-E_{b}\right)}{\partial \alpha_{i}} \frac{\alpha_{i}}{m_{I}}\left(\frac{c^{2}}{g} \frac{\nabla \alpha_{i}}{\alpha_{i}}\right)\right|_{A} \\
& -\left.\sum_{i} \frac{\partial\left(M_{n-p}-E_{b}\right)}{\partial \alpha_{i}} \frac{\alpha_{i}}{m_{I}}\left(\frac{c^{2}}{g} \frac{\nabla \alpha_{i}}{\alpha_{i}}\right)\right|_{B},
\end{aligned}
$$

siendo $i=\alpha, \Lambda_{Q C D}, \sin ^{2} \theta_{W}, G_{F} ; m_{I} \sim 0,931 \times 10^{3} \mathrm{AMeV}$;

$M_{n-p}=\sum_{t} \Delta M_{n-p}^{t}$ y $E_{b}=\sum_{t} E_{b}^{t}$, con $t=e m, s t, W$. Luego, haciendo uso de las ecs. (5.13), es fácil ver que $\alpha$ está relacionada con las diferencias de masa neutrón-protón 


\begin{tabular}{ccc}
\hline Par & $\Theta_{j}$ c/ 1 variables & $\Theta_{j}$ c/ 4 variables. \\
\hline$\Theta_{\alpha} \times 10^{10}$ & $0,029 \pm 0,692$ & $3,67 \pm 9,66$ \\
$\Theta_{\Lambda_{Q C D}} \times 10^{10}$ & $-0,075 \pm 0,253$ & $3,47 \pm 6,30$ \\
$\Theta_{\sin ^{2} \theta_{W}} \times 10^{2}$ & $0,022 \pm 0,024$ & $1,83 \pm 2,92$ \\
$\Theta_{G_{F}} \times 10^{8}$ & $0,084 \pm 0,089$ & $-6,14 \pm 10,06$ \\
\hline
\end{tabular}

Cuadro 5.4: Cotas superiores para las variaciones espaciales de las CF. La 1er columna muestra los resultados suponiendo que una sola variación viola al WEP, y la 2da columna representa los datos obtenidos suponiendo que todas las variaciones rompen el WEP.

(debido a que $\alpha$ aparece en la fórmula de Cottingham) y con la energía de ligadura electromagnética; y $\Lambda_{Q C D}$ lo está con todos los términos, ya que es proporcional a las masas e inversamente al radio nuclear, excepto con $\Delta M_{n-p}^{s t}$ como mostramos en la subsección 5.3.1. $\sin ^{2} \theta_{W}$ y $G_{F}$ tienen influencia en las contribuciones relacionadas con la interacción débil $\left(\Delta M_{n-p}^{W}\right.$ y $\left.E_{b}^{W}\right)$, y la constante de Fermi además interviene en $\Delta M_{n-p}^{s t}$. De esta manera, estamos en condiciones de ajustar los páramentros $\Theta_{j}=\frac{c^{2}}{g} \frac{\nabla \alpha_{j}}{\alpha_{j}}$ (variaciones espaciales de las constantes (SCV)) con la información de los experimentos de Eötvös de la Tabla 5.1. Los resultados se encuentran expresados en la Tabla 5.4.

$$
\left.\operatorname{Corr}\right|_{S C V}=\left(\begin{array}{lccc}
1 & 0,96 & 0,70 & -0,68 \\
0,96 & 1 & 0,87 & -0,85 \\
0,70 & 0,87 & 1 & -0,99 \\
-0,68 & -0,85 & -0,99 & 1
\end{array}\right)
$$

Examinando la matriz de correlación mostrada arriba, deducimos que variaciones de $\alpha$ y $\Lambda_{Q C D}$ están altamente correlacionadas entre ellas, en menor medida con la variación de $\sin ^{2} \theta_{W}$, mientras $\Theta_{G_{F}}$ tiene la menor correlación con los dos primeros parámetros. Por el otro lado, hay una enorme correlación entre $\Theta_{\sin ^{2} \theta_{W}}$ y $\Theta_{G_{F}}$. Igualmente, todas las correlaciones son muy altas ya que la más baja que se presenta es de 0.70. Mediante el estudio de las variaciones espaciales de las constantes fundamentales podemos determinar cotas inferiores para los rangos de longitud de campos 


\begin{tabular}{ccc}
\hline Par. & $l_{\alpha_{i}} \mathrm{c} / 1$ var. $(\mathrm{Mpc})$ & $l_{\alpha_{i}} \mathrm{c} / 4$ var. $(\mathrm{Mpc})$ \\
\hline$l_{\alpha} \times 10^{-5}$ & $1,026 \pm 24,489$ & $0,008 \pm 0,021$ \\
$l_{\Lambda_{Q C D}} \times 10^{-4}$ & $3,968 \pm 13,386$ & $0,086 \pm 0,156$ \\
$l_{\sin ^{2} \theta_{W}} \times 10^{3}$ & $1,353 \pm 1,476$ & $0,016 \pm 0,026$ \\
$l_{G_{F}} \times 10^{-2}$ & $3,54 \pm 3,75$ & $0,048 \pm 0,079$ \\
\hline
\end{tabular}

Cuadro 5.5: Límites inferiores para los rangos de longitud de campos escalares.

escalares quienes son los responsables de generar dichos cambios,

$$
\left(\frac{\nabla \alpha_{i}}{\alpha_{i}}\right)^{-1}=\frac{c^{2}}{g \Theta_{\alpha_{i}}}=l_{\alpha_{i}} .
$$

Las longitudes se encuentran en la Tabla 5.5. Los resultados muestran que los rangos de longitudes son más acotados cuando las variaciones se analizan por separado. Además, en los casos de las variaciones espaciales de $\alpha, \Lambda_{Q C D}$ y en menor medida en $G_{F}$, las cotas en las longitudes son bastante menos precisas que en la variación de $\sin ^{2} \theta_{W}$.

\subsubsection{Relación entre los $\Gamma$ y las variaciones de las constantes}

Ahora quisieramos conectar los parámetros de violación $\Gamma_{t}$ del Principio de Equivalencia con los valores que obtuvimos para $\Theta_{j}$ (tomando los casos donde las tres interacciones no cumplen el WEP y las cuatro variaciones tampoco lo hacen). Ya tenemos calculados $\boldsymbol{\eta}(A, B)=\boldsymbol{Q} . \boldsymbol{\Gamma}$ y $\boldsymbol{\eta}(A, B)=\boldsymbol{Y} . \boldsymbol{\Theta}$, con $\boldsymbol{Q}$ y $\boldsymbol{Y}$ las correspondientes matrices de diseño, ambas pesadas con los $\sigma_{j}$ de las medidas $\eta_{j}(A, B)$. De esta forma, haciendo una transformación, escribimos $\Gamma=\left\{\Gamma^{e m}, \Gamma^{s t}, \Gamma^{W}\right\}$ como una función de $\boldsymbol{\Theta}=\left\{\Theta_{\alpha}, \Theta_{\Lambda_{Q C D}}, \Theta_{\sin ^{2} \theta_{W}}, \Theta_{G_{F}}\right\}, \boldsymbol{\Gamma}=\boldsymbol{S} . \Theta$

$$
\boldsymbol{S}=\left(\begin{array}{lccc}
1,00 & 0,06 & 1,5 \times 10^{-8} & 0,10 \\
-4,26 \times 10^{-16} & 1,07 & 1,4 \times 10^{-8} & 0,09 \\
-7,15 \times 10^{-10} & 9,12 \times 10^{5} & 0,65 & 1,92 \times 10^{5}
\end{array}\right)
$$

Reemplazamos los $\Theta_{j}$ con los valores hallados gracias al ajuste, obteniendo la Tabla 5.6. Luego de hacer una comparación entre los resultados de la Tabla 5.6 con los de 


\begin{tabular}{ccc}
\hline Par & Valor & Incertidumbre \\
\hline$\Gamma^{e m} \times 10^{9}$ & 0,024 & 0,741 \\
$\Gamma^{s t} \times 10^{9}$ & 0,059 & 0,370 \\
$\Gamma^{W} \times 10^{3}$ & 0,420 & 0,935 \\
\hline
\end{tabular}

Cuadro 5.6: Ajuste de parámetros de la relaciøñ con SVC

la Tabla 5.3, podemos concluir que los datos calculados son compatibles entre sí.

$$
\left.\operatorname{Corr}\right|_{\Gamma S V C}=\left(\begin{array}{ccc}
1 & 0,99 & 0,94 \\
0,99 & 1 & 0,97 \\
0,94 & 0,97 & 1
\end{array}\right)
$$

De la matriz de correlación se desprende la intrincada relación entre los parámetros analizados $\Gamma^{W}, \Gamma^{e m}$ y $\Gamma^{s t}$, que en este caso se obtuvieron a partir de las variaciones espaciales de las constantes.

\subsection{Variaciones de las constantes de acoplamiento y el VEV del Higgs}

Nuestra intención ahora es de vincular las variaciones espaciales de $\alpha, \Lambda_{Q C D}$, $\sin ^{2} \theta_{W}$ y $G_{F}$, con las variaciones espaciales de las constantes de acoplamiento $\alpha_{1}$, $\alpha_{2}, \alpha_{3}$ y el valor de expectación en el vacío del campo de Higgs (VEV) $v$.

Las constantes de acoplamiento $\alpha_{1}, \alpha_{2}, \alpha_{3}$ provenientes de $\mathrm{U}(1), \mathrm{SU}(2)$ y $\mathrm{SU}(3)$ se encuentran unidos con nuestros observables mediante las ecuaciones,

$$
\begin{gathered}
\alpha=\alpha_{1} \sin ^{2} \theta_{W}, \quad G_{F}=\frac{\alpha_{1} \sqrt{2}}{8 M_{W}^{2}} \\
\tan ^{2} \theta_{W}=\frac{\alpha_{2}}{\alpha_{1}}, \quad \alpha_{3}=\left(\beta \ln \left(\mu^{2} / \Lambda_{Q C D}^{2}\right)\right)^{-1},
\end{gathered}
$$

con $\mu$ una energía de escala tal que en este caso, $\mu=M_{p}$ ( $M_{p}$ masa del protón) y $\Lambda_{Q C D} \approx 0,55 M_{p}$. La constante de Fermi es además función del VEV via,

$$
M_{W}^{2}=\frac{v^{2}}{2} \alpha_{1}, \quad G_{F}=\frac{1}{2 \sqrt{2} v^{2}} .
$$




\begin{tabular}{cc}
\hline Par & Valor \\
\hline$\Theta_{\alpha_{1}} \times 10^{2}$ & $-2,37 \pm 3,80$ \\
$\Theta_{\alpha_{2}} \times 10^{2}$ & $-2,37 \pm 3,80$ \\
$\Theta_{\alpha_{3}} \times 10^{10}$ & $5,82 \pm 10,54$ \\
$\Theta_{v} \times 10^{8}$ & $3,07 \pm 5,03$ \\
\hline
\end{tabular}

Cuadro 5.7: Cotas superiores para las variaciones espaciales de las ctes de acoplamiento y $v$.

El valor de expectación $v$, se determina mediante la minimización del potencial efectivo del campo de Higgs (los artículos $[3,46]$ hacen un análisis detallado, y se explicará un poco más sobre este mecanismo en la próxima subsección).

Luego, usamos los resultados obtenidos anteriormente, para poder así establecer los límites superiores para las variaciones de las constantes de acoplamiento y $v$ (Tabla 5.7) gracias a una transformación estadística, tomando por supuesto que todas las variaciones interactúan simultáneamente. La siguiente matriz de correlación indica una correlación extrema entre las variaciones de $\alpha_{1}, \alpha_{2}$ y $v$ mientras que $\alpha_{3}$ se encuentra un poco menos pero aún así es más que apreciable.

$$
\left.\operatorname{Corr}\right|_{C C V E V}=\left(\begin{array}{lccc}
1 & 1 . & -0,87 & -0,99 \\
1 & 1 . & -0,87 & -0,99 \\
-0,87 & -0,87 & 1 & 0,85 \\
-0,99 & -0,99 & 0,85 & 1
\end{array}\right)
$$

\subsubsection{Variaciones de masas elementales}

En una teoría del tipo $\lambda \phi^{4}$, las rupturas espontáneas de simetría (esto es el valor de expectación del vacío del campo involucrado) se traducen en "términos de masa" en los sectores del Lagrangiano donde se da el acoplamiento con el campo.

En el caso del mecanismo de Higgs ocurre exactamente lo mismo, siendo el valor de expectación del vacío del campo de Higgs $\langle\phi\rangle=v$. El Lagrangiano de un bosón escalar real de masa $\mu$ está dado por,

$$
L_{H i g g s}=\frac{1}{2} \partial_{\mu} \phi \partial^{\mu} \phi-\frac{1}{2} \mu^{2} \phi^{2}-\frac{1}{4} \lambda \phi^{4},
$$


con $\lambda>0, \phi=\left(\begin{array}{c}\phi^{+} \\ \phi^{0}\end{array}\right)=\left(\begin{array}{c}\phi_{1}+i \phi_{2} \\ \phi_{3}+i \phi_{4}\end{array}\right)$. Para $\mu^{2}>0$, el único mínimo del potencial se halla en $\phi=0$ (figura 5.1), mientras que para $\mu^{2}<0$ existen 2 mínimos dados por $\pm v= \pm \sqrt{\frac{-\mu^{2}}{\lambda}}= \pm \sqrt{\phi^{0^{2}+\phi^{+2}}}$ (figura 5.2). Estos dos "puntos", que minimizan al potencial en una dimensión, en realidad corresponden a un continuo de valores mínimos en $S U(2)$, siendo $\phi=0$ en este caso un punto inestable. Una buena descripción del mecanismo se obtiene a partir del estudio de una perturbación alrededor de uno de los "mínimos" $\left(\phi=\left(\begin{array}{c}0 \\ v+H\end{array}\right)\right)$ ya que, la elección de uno de ellos como valor de vacío $\left(\phi_{3}=\phi^{0}=v\right.$ y $\left.\phi_{j}=0, j=1,2,4\right)$ es la que genera la ruptura espontánea de la simetría. En aquellas teorías que contengan un bosón hipercargado y 3 bosones asociados al sector que depende del isospin, la ruptura espontánea de simetría le da masa a 3 de esos bosones manteniendo la masa nula del otro de ellos, transformando así a la representación original $S U_{L}(2) \times U_{Y}(1) \rightarrow S U_{\text {debil }}(2) \times U_{e m}(1)$ ( $Y=$ hipercargado, $L=$ isospin). En este esquema, los bosones que transforman a la interacción electrodébil, $W^{ \pm}$y $Z$, son proporcionales a $\langle\phi>$ mientras que el fotón es el que permanece sin masa. Tanto $Z^{0}$ como el fotón son combinaciones del bosón hipercargado original y del bosón neutro del grupo del isospin.

$$
M_{W^{ \pm}}=\frac{v g}{2}, \quad M_{Z}=\frac{M_{W}}{\cos \theta_{W}}=\frac{v\left(g^{\prime 2}+g^{2}\right)^{1 / 2}}{2},
$$

tanto $g$ como $g^{\prime}$ representan los acoplamientos de Gauge débil y electromagnético respectivamente, los cuales están relacionados entre sí mediante ángulo de mezcla $\theta_{W}, \tan ^{2} \theta_{W}=\frac{g^{\prime}}{g}$.

Respecto a los fermiones, se supone que el Lagrangiano de la partícula contiene acoplamientos de Yukawa con los bosones mediadores, siendo el mecanismo de Higgs el que les proporciona masa a los fermiones. El término fermiónico de masa $L_{\text {masa }}^{f}=-m_{f} \bar{f} f=-m_{f}\left[\bar{f}_{L} f_{R}+\bar{f}_{R} f_{L}\right]$ se excluye del Lagrangiano de una partícula ya que rompe con la invarianza de Gauge, pero una forma de dar masa a dicho tipo de partículas sin romper con la simetría de Gauge, es a través de los acoplamientos de Yukawa de los fermiones con el campo de Higgs $\phi$. Para el caso de los leptones, 


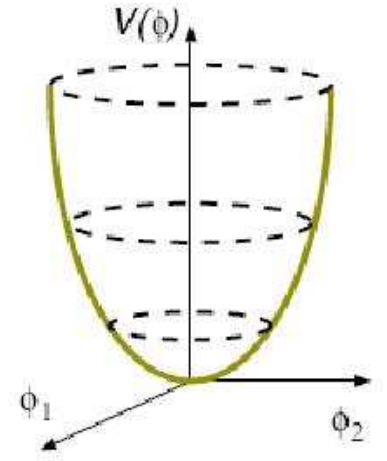

Figura 5.1: $\mu^{2}>0$

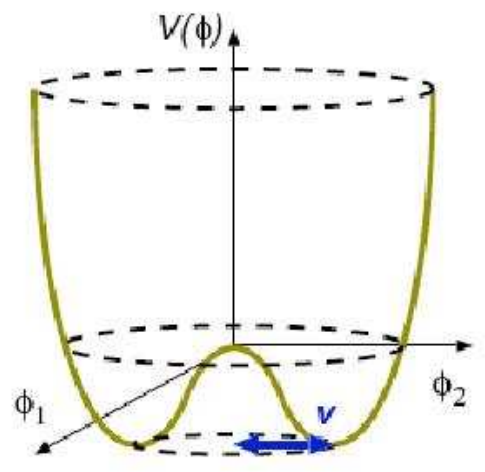

Figura 5.2: $\mu^{2}<0$

el lagrangiano está dado por,

$$
\begin{aligned}
L_{Y u k}^{\ell} & =-\lambda_{\ell} \frac{v+H}{\sqrt{2}}\left[\overline{\ell_{R}}(01)\left(\begin{array}{l}
\nu_{L} \\
\ell_{L}
\end{array}\right)+\left(\overline{\nu_{L} \bar{\ell}_{L}}\right)\left(\begin{array}{l}
0 \\
1
\end{array}\right) \ell_{R}\right], \\
& =-\lambda_{\ell} \frac{v}{\sqrt{2}} \bar{\ell} \ell-\lambda_{\ell} \frac{\bar{\ell} \ell}{\sqrt{2}} H
\end{aligned}
$$

Se puede inferir entonces que la masa de los leptones $m_{l}$ es proporcional al acoplamiento de Yukawa correspondiente $\lambda_{l}$ y al valor de expectación de vacío del campo de Higgs $v$.

Las masas de los quarks se generan de manera semejante a las de los leptones (siendo así proporcionales al correspondiente acoplamiento de Yukawa $\lambda_{q}$ y al VEV) , pero en este caso, se necesita de la construcción de un nuevo doblete del campo de Higgs para el miembro superior del doblete de los quarks,

$$
\phi_{C}=i \tau_{2} \phi^{*}=\left(\begin{array}{c}
-\bar{\phi}^{0} \\
\phi^{-}
\end{array}\right) \rightarrow \sqrt{\frac{1}{2}}\left(\begin{array}{c}
v+H \\
0
\end{array}\right)
$$

que se transforma de casi de igual manera que el doblete de Higgs tradicional, pero con una hipercarga débil opuesta. Luego, se opera de forma similar al caso anterior a partir de un Lagrangiano de Yukawa invariante de Gauge.

$$
m_{q}=\frac{\lambda_{q}}{\sqrt{2}} v, \quad m_{l}=\frac{\lambda_{l}}{\sqrt{2}} v .
$$




\begin{tabular}{cc}
\hline $\operatorname{Var} \frac{g}{c^{2}}$ & Valor \\
\hline$\frac{\nabla M_{Z}}{M_{Z}} \times 10^{2}$ & $-1,2 \pm 1,9$ \\
$\frac{\nabla M_{W}}{M_{W}} \times 10^{2}$ & $-1,2 \pm 1,9$ \\
$\frac{\nabla m_{q}}{m_{q}} \times 10^{8}$ & $3,07 \pm 5,03$ \\
$\frac{\nabla m_{l}}{m_{l}} \times 10^{8}$ & $3,07 \pm 5,03$ \\
\hline
\end{tabular}

Cuadro 5.8: Límites superiores para las variaciones espaciales de las masas elementales.

Las expresiones para las masas, que están dadas a partir de las constantes de acoplamiento de Gauge débil $g$ y electromagnético $g^{\prime}$, las podemos escribir en función de las constantes de acoplamiento anteriormente vistas $\alpha_{1}$ y $\alpha_{2}$ ya que $g=\sqrt{2 \alpha_{1}}$ y $g^{\prime}=\sqrt{2 \alpha_{2}}$. También reescribimos a las masas fermiónicas tal que, los correspondientes potenciales de Yukawa cumplen $\lambda_{q}=\sqrt{2} \varpi^{2}$ y $\lambda_{l}=\sqrt{2} \kappa^{2}$,

$$
\begin{gathered}
M_{W}^{2}=\frac{v^{2} \alpha_{1}}{2}, \quad M_{Z}^{2}=\frac{v^{2}\left(\alpha_{1}+\alpha_{2}\right)}{2}, \\
m_{q}=\varpi^{2} v, \quad m_{l}=\kappa^{2} v .
\end{gathered}
$$

Por lo tanto, de haber encontrado cotas para los cambios en el espacio de las constantes de acoplamiento y de $v$ podemos llegar a conseguir límites superiores para las variaciones espaciales de las masas de partículas elementales. Los resultados están reflejados en la Tabla 5.8, mientras que la matriz de correlación es,

$$
\text { Corr }\left.\right|_{\text {masses }}=\left(\begin{array}{lccc}
1 & 1 & -0,99 & -0,99 \\
1 & 1 & -0,99 & -0,99 \\
-0,99 & -0,99 & 1 & 1 \\
-0,99 & -0,99 & 1 & 1
\end{array}\right)
$$

Nuevamente queda claro que el grado de correlación es extremo. 


\section{Capítulo 6}

\section{Conclusiones}

Aquí presentaremos nuestras conclusiones a partir de los resultados que fuimos obteniendo en los capítulos 3, 4, 5. En esta tesis quisimos principalmente, hacer un estudio exhaustivo de la teoría propuesta por Bekenstein. Para llevar a cabo esto, modificamos en ella el modelo de partícula utilizado a fin de tener un sistema más realista, tratando de abordar todos los "frentes" posibles de análisis tales como, la contribución del campo electromagnético al campo escalar acoplado, las fuerzas generadas sobre una densidad de carga estática acotada y el intercambio de energía entre la materia y los campos electromagnético y escalar $\psi$. Además, examinamos los parámetros que describen a las violaciones del Principio de Equivalencia debido a contribuciones de las interacciones fuerte, electromagnética y débil utilizando los últimos datos obtenidos a partir de experimentos Eötvös; y sus vinculaciones con los límites superiores de las variaciones espaciales de constantes fundamentales $\alpha$,

$\lambda_{Q C D}, \sin ^{2} \theta_{W}$ y $G_{F}$. Estas últimas cotas las utilizamos para establecer restricciones a las variaciones espaciales de las constantes de acoplamiento de Gauge, al valor de expectación del Higgs y a las masas de partículas elementales.

- Al momento de examinar las contribuciones electromagnéticas, observamos que si bien no podemos afirmar ni contradecir la idea de que el campo eléctrico no contribuye al campo $\psi$ (ya que para examinar bien el término relacionado con la masa es necesario cuantizar los campos electromagnético y dilatónico $\psi$, y esto se escapa de nuestra propuesta semi-clásica), y por ende suponemos que se cumple lo predicho por Bekenstein; existen otras contribuciones que provienen 
de las fluctuaciones cuánticas de la materia. Éstas, producen corrientes que generan energía magnética, pudiendo llegar a producir una violación del Principio de Equivalencia. Pero dado que este desarrollo puede tomarse como límite a bajas energías de modelos de cuerdas, nuestra conclusión debe ser tomada con cuidado.

- Por otra parte, el intercambio de energía con la materia en las teorías alternativas con nuevos campos escalares acoplados, como la teoría estudiada en este trabajo, es un tema poco desarrollado hasta hoy. Utilizando las ecuaciones de campo y las hipótesis propuestas en el modelo de Bekenstein pudimos estimar la transferencia de energía entre la materia y los campos. La hipótesis 8 del capítulo 2 es clave, ya que afirma que el tensor energía-momento de la materia es la cantidad que tiene que ser añadida al sector de campo a fin de que la divergencia del tensor total sea libre. También hemos asumido que la materia oscura es eléctricamente neutra, despreciamos el movimiento de la materia de los cuerpos cargados que hemos considerado, y hallamos que una característica dinámica de la carga eléctrica hace que la energía electromagnética atómica forme parte de la energía interna del sistema. Encontramos una contribución "extra" al calor actual, además de la refrigeración de la materia, que viene dada por la variación de tiempo en el campo escalar y el contenido magnético de la materia; que puede ser testeada para comprobar la validez de la teoría. Los datos observacionales utilizados para dicha verificación fueron obtenidos a partir de aspectos geotermales de la Tierra y planetas exteriores hallando cotas que son modelo independientes para la variación temporal de $\alpha$ del mismo orden que las cotas de los más precisos relojes atómicos y sólo un orden de magnitud menos riguroso que la cota de Oklo. De la comparación del modelo con los experimentos llegamos a la conclusión de que el modelo debe ser descartado.

- Por último, analizamos las contribuciones de las diferencias de masa neutrónprotón y de las energías de enlace de las interacciones fundamentales para poder determinar los párametros de violación $\Gamma$ del Principio de Equivalencia utilizando los más modernos datos provenientes de experimentos Eötvös. Las cotas que determinamos en este trabajo son uno o dos órdenes más precisas 
que las encontradas hasta este momento. Estudiamos también las variaciones espaciales de algunas de las constantes fundamentales trabajando cerca del límite quiral, y luego relacionamos las cotas que establecimos para ellas con los parámetros $\Gamma$, y los cambios en las constantes $\alpha_{1}, \alpha_{2}, \alpha_{3}, v$ y las masas elementales; e inclusive, las utilizamos para estimar los rangos de longitud "permitidos" de campos escalares que podrían generar variaciones espaciales. Los elementos de las matrices de correlación de los ajustes en los casos investigados son tan elevados que se dificulta muchísimo el estudio de las posibles variaciones espaciales en los parámetros del Modelo Estandard elegidos. Porque entonces, cabe la posibilidad de la existencia de violaciones debidas a las distintas variaciones que se superpongan entre sí, escondiéndose del "observador" y no permitiendo la determinación de mejores cotas. Por lo tanto, nos es necesario tener que ajustar un número menor de parámetros a fin de poder disminuir las correlaciones y conseguir así cotas más fiables. 


\section{Apéndice A}

\section{Tratamiento semiclásico del modelo de Thomas -Fermi}

El modelo puede considerarse como semiclásico debido a que, si bien toma varias ideas de la mecánica cuántica como;

- la estadística propuesta por Fermi, tal que todos estados con menor energía que la máxima se encuentran ocupados;

- el Prinpicio de Incerteza, cada celda del espacio de fases puede albergar hasta 2 electrones con spines opuestos;

utiliza las aproximaciones cuasi-clásicas para funciones de onda de de Broglie [114].

En mecánica clásica podemos representar toda la información sobre el estado de un sistema como un punto en el espacio de fases $(q, p)$. Sin embargo, en un sistema real, esto no es posible. Por tanto, para poder caracterizarlo es necesario hacer un promedio de los puntos del espacio de fases como una variable estocástica y realizar una distribución de probabilidades. Ésta evolucionará en el espacio de fases debido a la evolución de cada uno de sus puntos y se denomina densidad en el espacio de fases clásico $\rho(q, p)$, perdiendo el una parte determinismo total del modelo clásico.

Si las longitudes de onda de de Broglie de las partículas son pequeñas comparadas a las dimensiones características que determinan las condiciones del problema, entonces las propiedades del sistema se aproximan a las clásicas. 
Escribimos la ecuación de Schrödinger (en este caso E está representando a la energía y U al potencial) haciendo la sustitución,

$$
\sum_{a} \frac{\hbar^{2}}{2 m_{a}} \Delta_{a} \Psi+(E-U) \Psi=0 \quad \Psi=e^{(i / \hbar) \Gamma},
$$

tal que la ecuación que queda para $\Gamma$ es,

$$
\sum_{a} \frac{\left(\nabla_{a} \Gamma\right)^{2}}{2 m_{a}}-\sum_{a} \frac{i \hbar}{2 m_{a}} \Delta_{a} \Gamma=E-U
$$

Al estar trabajando en un sistema semiclásico, podemos desarrollar en serie de potencias de $\hbar$,

$$
\Gamma=\Gamma_{0}+(\hbar / i) \Gamma_{1}+(\hbar / i)^{2} \Gamma_{2}+\ldots
$$

Siendo el potencial central, y estudiando el movimiento para una partícula, la ecuación se reduce a,

$$
\frac{\Gamma^{\prime 2}}{2 m}-i \hbar \frac{\Gamma^{\prime \prime}}{2 m}=E-U(r)
$$

A orden 0 ,

$$
\frac{\Gamma_{0}^{\prime 2}}{2 m}=E-U(r) \quad \Gamma_{0}= \pm \int(2 m[E-U(r)])^{1 / 2} d r
$$

donde el integrando es justamente el impulso clásico $p$. A primer orden, la ecuación queda,

$$
\begin{gathered}
\Gamma_{0}^{\prime} \Gamma_{1}^{\prime}+\frac{\Gamma_{0}^{\prime \prime}}{2}=0 \\
\Gamma_{1}^{\prime}=-\frac{\Gamma_{0}^{\prime \prime}}{2 \Gamma_{0}^{\prime}}=-\frac{p^{\prime}}{2 p} \quad \Gamma_{1}=-\frac{\log p}{2} .
\end{gathered}
$$

Sustituyendo en las expresiones (A.1) y (A.3), la función de onda $\Psi$ queda de la forma,

$$
\Psi=C_{1} p^{-1 / 2} e^{(i / \hbar) \int p d r}+C_{2} p^{-1 / 2} e^{-(i / \hbar) \int p d r}
$$

Los siguientes órdenes nos darán términos de orden superior respecto de $\hbar$ en las exponenciales, pero en general, el primer orden ya es suficiente. 
Como la función de onda $\Psi$ posee en ambos términos el factor $p^{-1 / 2}$, la probabilidad de hallar a la partícula en puntos que se encuentran en la región $r$ y $r+d r$ está determinada por $|\Psi|^{2}$, es inversamente proporcional a $p$. Esto es esperable en una partícula "semiclásica" ya que clásicamente, el tiempo que pasa la partícula en $d r$ es inversamente proporcional al impulso.

En las regiones del espacio donde $E<U$ (clásicamente inaccesibles), el impulso es una función imaginaria pura por lo tanto los exponentes son reales y la función de onda será:

$$
\Psi=\frac{C_{1}^{\prime}}{(|p|)^{1 / 2}} e^{-(1 / \hbar) \int|p| d r}+\frac{C_{2}^{\prime}}{(|p|)^{1 / 2}} e^{(1 / \hbar) \int|p| d r}
$$

Podemos deducir la condición que determina los niveles cuánticos de energía para el caso cuasi-clásico gracias al estudio de las condiciones en los límites que deben cumplir las funciones de onda. Sea el dominio accesible clásicamente, $b \leq x \leq a$, las condiciones de contorno nos llevan a que las funciones deben tener la forma,

$$
\begin{aligned}
& \Psi_{b}=\frac{C}{p^{1 / 2}} \sin \left[\frac{1}{\hbar} \int_{b}^{x} p d x+\frac{\pi}{4}\right] \\
& \Psi_{a}=\frac{C^{\prime}}{p^{1 / 2}} \sin \left[\frac{1}{\hbar} \int_{x}^{a} p d x+\frac{\pi}{4}\right] .
\end{aligned}
$$

Dado que ambas deben coincidir en todas la región, la suma de las fases, debe ser un múltiplo enetro de $\pi$. Siendo $C=(-1)^{n} C^{\prime}$ y extendiendo la integral a todo un período del movimiento se cumple la Regla de cuantificación de Bohr-Sommerfield,

$$
\oint p d x=2 \pi \hbar\left(n+\frac{1}{2}\right)
$$

donde $n$ es igual al número de ceros de la función de onda. De la normalización de la función a 1 , obtenemos

$$
\Psi=\sqrt{\frac{2 \omega}{\pi v}} \sin \left[\frac{1}{\hbar} \int_{b}^{x} p d x+\frac{\pi}{4}\right]
$$

tal que $\omega=2 \pi / T$ es la frecuencia del movimiento periódico clásico y es función de la energía (diferente en cada nivel). 
Por otra parte, la integral $\oint p d x$ es el área limitada por la trayectoria cerrada clásica en el espacio de las fases $(x, p)$ de la partícula. Por lo tanto, podemos dividir esta área en $n$ celdas de área $2 \pi \hbar$ cada una, siendo $n$ además el número de estados cuánticos con energías que no superan el valor dado de energía que corresponde al valor de la trayactoria. De esta manera, el número de estados que corresponden al elemento de volumen $\Delta p \Delta x$ del espacio de fases es $\Delta p \Delta x / 2 \pi \hbar$ y trabajando en forma similar observamos que la diferencia de energía entre dos niveles es $\Delta E=2 \pi \hbar / T=$ $\hbar \omega$.

Todos estos resultados pueden generalizarse para sistemas de varias partículas con varios grados de libertad que efectuán un movimiento finito tal que el problema de mecánica "clásica" permite una separación completa de variables en el método de Hamilton-Jacobi, ya que luego de ella la condición queda,

$$
\oint p_{i} d q_{i}=2 \pi \hbar\left(n_{i}+\gamma_{i}\right) .
$$

La integral se extiende a un período de la variación de la coordenada generalizada $q_{i}$, y $\gamma_{i}$ depende del caracter de las condiciones de contorno para cada grado de libertad considerado.

En un caso general de un movimiento multidimensional cualquiera (no cuasiperiódico), se modifica el concepto de número cuántico $n_{i}$ por el de "celdas" en el espacio de fases. Así en un sistema con s grados de libertad, a cada elemento del espacio de fases le corresponden $\Delta N$ estados cuánticos, $\Delta N=\Delta q_{1} \cdots \Delta q_{s} \Delta p_{1} \cdots \Delta p_{s} /(2 \pi \hbar)^{s}$.

\section{A.1. Ecuación de Thomas-Fermi}

En átomos complejos con números cuánticos principales altos, es posible utilizar el tratamiento cuasi-clásico y describir a los estados de los electrones como las celdas del espacio de fases descriptas anteriormente.

El volumen del espacio de fases $d V_{f}$ que corresponde a los electrones cuyo impulso es menor que $p(\vec{r})$ y están en el elemento de volumen $d V$ del espacio físico es igual a $\frac{4 \pi}{3} p^{3}(\vec{r}) d V$. A $d V_{f}$ le correponden $d N_{f}$ celdas tal que, $d N_{f}=\frac{4 \pi p^{3}(\vec{r})}{3(2 \pi \hbar)^{3}} d V$, que representa al número estados posibles en los se pueden encontrar $2 d N_{f}$ electrones. En el estado normal del átomo, los electrones deben llenar las celdas que corresponden a un $p(\vec{r})$ 
desde cero hasta el máximo $p_{F}(\vec{r})$. Luego, $n(\vec{r})$ la densidad número de electrones la podemos determinar mediante número de electrones que está dado por $n(\vec{r}) d V$,

$$
n(\vec{r})=\frac{p_{F}^{3}(\vec{r})}{3 \pi^{2} \hbar^{3}} .
$$

Consecuentemente, podemos escribir a la energía cinética máxima de los electrones en un punto $\vec{r}$ como,

$$
E_{\text {cin }}(\vec{r})=\frac{p_{F}^{2}(\vec{r})}{2}=\frac{1}{2}\left(n(\vec{r}) 3 \pi^{2} \hbar^{3}\right)^{2 / 3},
$$

siendo la energía total del electrón $E_{T}=\frac{p^{2}(\vec{r})}{2}-\phi_{C}(\vec{r}), \phi_{C}(\vec{r})$ el potencial electrostático que se anula en el infinito. $E_{T}$ debe ser negativa ya que si no lo es, el electrón escaparía al infinito. Sea $-\phi_{O C}(\vec{r})$ el máximo de la energía total en cada punto tal que,

$$
\begin{gathered}
\frac{p_{F}^{2}(\vec{r})}{2}=\phi_{C}(\vec{r})-\phi_{0 C}(\vec{r}), \\
n(\vec{r})=\frac{\left[2\left(\phi_{C}(\vec{r})-\phi_{0 C}(\vec{r})\right)\right]^{3 / 2}}{3 \pi^{2} \hbar^{3}} .
\end{gathered}
$$

Para $\phi_{C}(\vec{r})=\phi_{0 C}(\vec{r})$ y $\phi_{C}(\vec{r})<\phi_{0 C}(\vec{r}), n(\vec{r})$ se anula, siendo $\phi_{C}(\vec{r})=\phi_{0 C}(\vec{r})$ el límite del átomo. Pero fuera de una distribución central de cargas cuya carga total es 0 no existe campo, por lo tanto, en la frontera de un átomo neutro $\phi_{C}(\vec{r})=0 \mathrm{y}$ en ese caso, $\phi_{0 C}(\vec{r})=0$. Para un ion, $\phi_{0 C}(\vec{r}) \neq 0$ pero sí es constante.

Este tratamiento puede generalizarse totalmente para el caso del núcleo atómico. Luego utilizando la ecuación electrostática de Poisson,

$$
\nabla^{2} \phi_{C}(\vec{r})=-4 \pi Q_{0} n(\vec{r})
$$

siendo entonces $\rho_{c}(\vec{r})=Q_{0} n(\vec{r})$ y $\rho_{m}(\vec{r})=m_{0} n(\vec{r})$ las densidades de carga y masa respectivamente. 


\section{Bibliografía}

[1] E. G. Adelberger. Classical and Quantum Gravity, 18:2397, 2001.

[2] E. G. Adelberger, J. H. Gundlach, B. R. Heckel, S. Hoedl, y S. Schlamminger. Prog. in Part. and Nuc. Phys., 62:102-134, 2009.

[3] V. Agrawal, S. M. Barr, J. F. Donoghue, y D. Seckel. Phys. Rev. D, 57:54805492, 1998.

[4] W. M. Alberico, S. M. Bilenky, C. Giunti, y K. M. Graczyk. Phys. Rev. C, 79(6):065204, 2009.

[5] P. Alberto, S. S. Avancini, y M. Fiolhais. Int. J. Mod. Phys. E, 14:1171-1196, 2005.

[6] A. Albrecht y J. Magueijo. Phys. Rev. D, 59:043516, 1999.

[7] N. Argaman y G. Makov. American Journal of Physics, 68:69-79, 2000.

[8] S. Baeßler, B. R. Heckel, E. G. Adelberger, J. H. Gundlach, U. Schmidt, y H. E. Swanson. Phys. Rev. Lett., 83:3585, 1999.

[9] J. Bahcall, C. L. Steinhardt, y D. Schlegel. Astrophys. J., 600:520, 2004.

[10] J. N. Bahcall y M. Schmidt. Phys. Rev. Lett., 19:1294, 1967.

[11] J. Barrow y D. Shaw. Phys. Rev. D, 78:067304, 2008.

[12] J. D. Barrow y J. Magueijo. Phys. Rev. D, 72:043521, 2005.

[13] R. A. Battye, R. Crittenden, y J.Weller. Phys. Rev. D, 63:043505, 2001. 
[14] T. Becher y H. Leutwyler. Eur. Phys. J. C, 9:643-671, 1999.

[15] J. D. Bekenstein. Phys. Rev. D, 25:1527, 1982.

[16] J. D. Bekenstein. Phys. Rev. D, 66:123514, 2002.

[17] J. Bernstein, L. Brown, y G. Feinberg. Rev. Mod. Phys., 61:25, 1989.

[18] S. Blatt, A. Ludlow, et al. Phys. Rev. Lett., 100:140801, 2008.

[19] V. B. Braginski y V. I. Panov. Sov. Phys. J. E. T. P., 34:463, 1972.

[20] P. Brax y A. C. Davis. Journal of High Energy Physics, 7:5, 2001.

[21] K. Capelle. eprint arXiv:cond-mat/0211443, 2002.

[22] N. Chamoun y H. Vucetich. Phys. Lett. B, 541:291-297, 2002.

[23] S. A. Chin y J. D. Walecka. Phys. Lett. B, 52:24-28, 1974.

[24] A. Chodos y S. Detweiler. Phys. Rev. D, 21:2167, 1980.

[25] H. R. Christiansen, L. N. Epele, H. Fanchiotti, y C. A. García Canal. Physics Letters B, 267:164-166, 1991.

[26] M. A. Clayton y J. W. Moffat. Phys. Lett. B, 460:263, 1999.

[27] M. A. Clayton y J. W. Moffat. Phys. Lett. B, 477:269, 2000.

[28] M. A. Clayton y J. W. Moffat. Phys. Lett. B, 177:506, 2001.

[29] G. Clément y J. Stern. Nuclear Physics A, 539:685-692, 1992.

[30] G. Colangelo, J. Gasser, y H. Leutwyler. Nuc. Phys. B, 603:125-179, 2001.

[31] W. N. Cottingham. Annals of Physics, 25:424-432, 1963.

[32] T. Damour. Gravity, Equivalence Principle and Clocks. Gr-qc/9711060.

[33] T. Damour. Classical and Quantum Gravity, 13:33, 1996.

[34] T. Damour y F. Dyson. Phys. Rev. B, 480:37, 1996. 
[35] T. Damour, F. Piazza, y G. Veneziano. Phys. Rev. Lett., 89:2081601, 2002.

[36] T. Damour y A. M. Polyakov. Nuc. Phys. B, 95:10347, 1994.

[37] T. Damour y A. M. Polyakov. Gen. Rel. Grav., 26:1171, 1994. Gr-qc/9411069.

[38] T. Damour y A. M. Polyakov. Nucl. Phys. B, 423:532, 1994. Hep-th/9401069.

[39] J. H. Davies y R. Davies. Solid Earth, 1:5, 2010.

[40] T. Dent. Phys. Rev. Lett., 101:041102, 2008.

[41] T. Dent. Phys. Rev. Lett., 101(4):041102, 2008.

[42] R. H. Dicke. Relativity,groups and topology. deWitt, C. and deWitt, B. ed., Gordon and Breach, New York, 1964.

[43] P. A. M. Dirac. The cosmological constants. Nature, 139:323, 1937.

[44] P. A. M. Dirac. A new basis for cosmology. Proc. Roy. Soc. Lon. A, 165:199208, 1938.

[45] J. F. Donoghue. Phys. Rev. C, 74(2):024002, 2006.

[46] J.F. Donoghue, K. Dutta, A. Ross, y M. Tegmark. Phys. Rev. D, 81(7):073003, 2010.

[47] G.R. Dvali y M. Zaldarriaga. Phys. Rev. Lett., 88:091303, 2002.

[48] F. Dyson. Phys. Rev.Lett., 19:1291, 1966.

[49] R. Von Eötvös, D. Pekar, y E. Fekete. Ann. Physik., 68:11, 1922.

[50] L. Epele, H. Fanchiotti, C. García Canal, y R. Méndez Galain. En H. M. Nussenzveig and R. A. Salmeron, ed., Frontier Phisics:Essays in Honour of Jayme Tiomno S. MacDowell. 1991.

[51] S. Baessler et al. Phys. Rev. Lett., 83:3585, 1999.

[52] E. Fermi. Rend. Acad. Maz. Lancei, 6:602, 1927. 
[53] R. Fernandez y A. Reisenegger. Ap. J., 625:291, 2005.

[54] A. Filin, V. Baru, E. Epelbaum, J. Haidenbauer, C. Hanhart, A. Kudryavtsev, y U.G. Meißner. Phys. Lett. B, 681:423-427, 2009.

[55] M. Fischer et al. Phys. Rev. Lett., 92:230802, 2004.

[56] T. M. Fortier et al. Phys. Rev. Lett., 96:070801, 2006.

[57] J. J. Fortney y W. B. Hubbard. Ap.J., 68:1039, 2004.

[58] Fujii et al. Nuc. Phys. B, 573:377, 2000.

[59] R. J. Furnstahl, B. D. Serot, y H. B. Tang. Nuc. Phys. A, 615:441-482, 1997.

[60] S. Galster, H. Klein, J. Moritz, K. H. Schmidt, D. Wegener, y J. Bleckwenn. Nuc. Phys. B, 32:221-237, 1971.

[61] G. Gamow. Phys. Rev. Lett, 19:759, 1967.

[62] J. Gasser y H. Leutwyler. Phys. Rep., 87:77-169, 1982.

[63] J. Harris. Phys. Rev. B, 31:1770-1779, 1985.

[64] M. P. Haugan. Ann. Phys., 118, 1979.

[65] M. P. Haugan y C. M. Will. Phys. Rev. Lett., 37:1-4, 1976.

[66] M. P. Haugan y C. M. Will. Phys.Rev.D, 15:2711-2720, 1977.

[67] J.D. Barrow H.B. Sandvik y J.Magueijo. Phys. Rev. Lett., 88:031302, 2002.

[68] W. B. Hubbard. Icarus, 35:177, 1978.

[69] P. G. J. Irwin. Giant planets of our solar system: an introduction. PraxisSpringer, Chichester-UK, 2006.

[70] P. Jain, R. Johnson, N. W. Park, J. Schechter, y H. Weigel. Phys. Rev. D, 40:855-865, 1989.

[71] A. M. Jessop. Thermal geophysics. Elsevier, Amsterdam, 1990. 
[72] P. Jofré, A. Reisenegger, y R. Fernandez. Phys.Rev.Lett., 97:131102, 2006.

[73] Th. Kaluza. Sitzungsber.Preuss. Akad.Wiss., K1:966, 1921.

[74] A. A. Kehagias y E. Kiritsis. Journal of High Energy Physics, 11:22, 1999.

[75] G.M. Keiser y J.E. Faller. In Proc. $2^{n} d$ Marcel Grossman Meeting on General Relativity. R.Ruffini ed., 1982.

[76] O. Klein. Z. Phys., 37:895, 1926.

[77] W. Kohn y L. J. Sham. Phys. Rev., 140:1133-1138, 1965.

[78] L. Kraiselburd, M. Miller Bertolami, P. Sisterna, y H. Vucetich. Astron. and Astrophys., 529:A125, 2011.

[79] L. Kraiselburd y H. Vucetich. Int. J. Mod. Phys. E, 20:101, 2011.

[80] L. Kraiselburd y H. Vucetich. ArXiv e-prints, siendo evaluado por Phys. Lett. $B, 2011$.

[81] L. Kraiselburd y H. Vucetich. en producción, 2011.

[82] S. Labrosse y C. Jaupart. Earth and Planetary Science Letters, 260:465, 2007.

[83] L. D. Landau y E. M. Lifschitz. Fluid Mechanics, 2nd EDITION. Elsevier, Oxford, 1987.

[84] L.D. Landau y E.M. Lifshitz. Teoría clásica de los campos. Reverté, 1972.

[85] L.D. Landau y E.M. Lifshitz. Mecánica cuántica no relativista. Reverté, 1978.

[86] S. J. Landau. La variación temporal y espacial de las constantes fundamentales: Cotejo entre teorías y datos geofísicos y astronómicos. Tesis Doctoral, Facultad de Ciencias Astronómicas y Geofísicas; Universidad Nacional de La Plata, 2002.

[87] S. J. Landau, D. D. Harari, y M. Zaldarriaga. Phys. Rev. D, 63:0835, 2001.

[88] S. J. Landau, M. E. Mosquera, C. G. Scóccola, y H. Vucetich. Phys. Rev. D, 78:083527, 2008. 
[89] S. J. Landau y H. Vucetich. Astrophys. J., 570:463, 2002.

[90] K. R. Lang. The Cambridge Guide to the Solar System. C. U. P., CambridgeLondon, 2003.

[91] H. Leutwyler. On the Foundations of Chiral Perturbation Theory. Ann. Phys., 235:165-203, 1994.

[92] Levshakov y et al. Mon.Not.R.Astron.Soc., 333:373, 2002.

[93] B. Li, D. F. Mota, y J. D. Barrow. 2010. ArXiv:1009.1396v1 [astro-ph.CO].

[94] K. Maeda. Mod. Phys. Lett. A, 31:243, 1988.

[95] B. Martin. Nuclear and Particle Physics, An Introduction, 2nd Edition. John Wiley and Sons, Inc, New York, 2009.

[96] S.J. Landau M.E. Mosquera, C.G. Scoccola y H. Vucetich. Astron. Astrophys., 478:675, 2008.

[97] U. G. Meiszner. Nuc. Phys. A, 666:51, 2000.

[98] R. Méndez Galain. Tesis Doctoral, Facultad de Ciencias Exactas; Universidad Nacional de La Plata, 1989.

[99] G. A. Miller, A. K. Opper, y E. J. Stephenson. Ann. Rev. of Nuc. and Part. Science, 56:253-292, 2006.

[100] C. W. Misner, K. S. Thorne, y J. A. Wheeler. Gravitation. W. H. Freeman, 1973.

[101] M. E. Mosquera y O. Civitarese. Astron. and Astrophys., 520:A112, 2010.

[102] N. C. Mukhopadhyay. ArXiv Nuclear Theory e-prints. Nucl-th/9810039.

[103] M. T. Murphy, J. K. Webb, y et al. Mon.Not.R.Astron.Soc., 327:1208, 2001.

[104] M. T. Murphy, J. K. Webb, y et al. Mon.Not.R.Astron.Soc., 327:1237, 2001. 
[105] M. T. Murphy, J. K. Webb, y V. V. Flambaum. Mon.Not.R.Astron.Soc., 345:609, 2003.

[106] M. J. Musolf, Donnelly, y et. al. Phys. Rep., 239:1-178, 1994.

[107] K. Nordtvedt. Phys. Rev. Lett., 65:953, 1990.

[108] NPLQCD Collaboration, S. R. Beane, K. Orginos, y M. J. Savage. Nuc. Phys. $B, 768: 38-50,2007$.

[109] B. Y. Park y M. Rho. Phys. Rev. D, 41:310-314, 1990.

[110] J. C. Pearl, B. J. Conrath, R. A. Hanel, y J. A. Pirraglia. Icarus, 84:12, 1990.

[111] E. Peik, B. Lipphardt, H. Schnatz, T. Schneider, y Chr. Tamm. Phys. Rev. Lett., 96:170801, 2004.

[112] C. F. Perdrisat, V. Punjabi, y M. Vanderhaeghen. Progress in Particle and Nuclear Physics, 59:694-764, 2007.

[113] M. E. Peskin y D. V. Schroeder. An introduction to quantum field theory. Addison-Wesley Pub. Co., New York, 1995.

[114] A. Postnikov. Lectures on the Density Functional Theory, Chap. 1, ThomasFermi method. Paul Verlaine University - Institut de Chimie, Physique et Materiaux, Laboratoire de Physique des Milieux Denses, France. URL http://www .home. uni-osnabrueck. de/apostnik/lectures.html.

[115] J. D. Prestage, R. L. Tjoelker, y L. Maleki. Phys. Rev. Lett., 74:3511, 1995.

[116] M. Procura, T.R. Hemmert, y W. Weise. Phys. Rev. D, 69(3):034505, 2004.

[117] M. Procura, B. U. Musch, T. Wollenweber, T. R. Hemmert, y W. Weise. Phys. Rev. D, 73(11):114510, 2006.

[118] A. Reisenegger. Ap. J., 442:749, 1995.

[119] P. G. Roll, R. Krotkov, y R. H. Dicke. Ann. Phys., 26:442, 1964.

[120] T. Rosenband et al. Science, 319:1808, 2008. 
[121] J. J. Rusnak y R. J. Furnstahl. Nuc. Phys. A, 627:495-521, 1997.

[122] S. Schlamminger, K. Y. Choi, y et al. Phys. Rev. Lett., 100:041101, 2008.

[123] C. Scóccola, M. E. Mosquera, S. J. Landau, y H. Vucetich. Astrophys. J., 681:737, 2008.

[124] B. D. Serot y J. D. Walecka. Adv. Nuc. Phys., 16:1, 1986.

[125] B. D. Serot y J. D. Walecka. Int. J. of Mod. Phys. E, 6:515-631, 1997.

[126] P. Sisterna y H. Vucetich. Phys. Rev. D, 41:1034, 1990.

[127] M. Smolliar. Science, 271:1099, 1996.

[128] Y. Sortais y et al. Physica Scripta, T95:50, 2000.

[129] P. M. Stevenson. Ann.of Phys., 132:383-403, 1981.

[130] Y. Su, B. R. Heckel, E. G. Adelberger, y et al. Phys. Rev. D, 50:3614, 1994.

[131] S. Stern T. Dent y C. Wetterich. Phys. Rev. D, 79:083533, 2009.

[132] E. Teller. On the change of physical constants. Phys. Rev., 73:801, 1948.

[133] L. H. Thomas. Proc. of the Cam. Phil. Soc., 23:542, 1927.

[134] J. P. Uzan. Rev. Mod. Phys., 75:403, 2003. hep-ph/0205340.

[135] J. P. Uzan. Liv. Rev. in Relativity, 14:2, 2011.

[136] H. Vucetich. Bol. Acad. Na. Cs., 61:1, 1996.

[137] J. D. Walecka. Ann. Phys., 83:491-529, 1974.

[138] J. K. Webb, V. V. Flambaum, y et al. Phys Rev. Lett., 82:884, 1999.

[139] J. K. Webb, J. A. King, M. Murphy, y et al. ArXiv e-prints 1008.3907, 2010.

[140] J. K. Webb, M. T. Murphy, y et al. Phys. Rev. Lett., 87:091301, 2001.

[141] S. Weinberg. Gravitation and Cosmology. Wiley, 1972. 
[142] S. Weinberg. Phys. Lett. B, 125:265, 1983.

[143] S. Weinberg. The quantum theory of fields, Volumen 1. Cambridge University Press, Cambridge, 1996.

[144] V. A. Weisskopf. Phys. Rev., 56:72, 1939.

[145] C. M. Will. Theory and Experiment in Gravitational Physics. C.U.P, 1981.

[146] J. G. Williams, S. G. Turyshev, y D. H. Boggs. Phys. Rev. Lett., 93(26):261101, 2004.

[147] D. Youm. Phys. Rev. D, 63:125011, 2001.

[148] X. H. Zhong y P. Z. Ning. Phys. Rev. C, 75(5):055206, 2007. 